\title{
$p$-ADIC FAMILIES OF MODULAR FORMS AND $p$-ADIC ABEL-JACOBI MAPS
}

\author{
MATTHEW GREENBERG AND MARCO ADAMO SEVESO
}

\begin{abstract}
We show that $p$-adic families of modular forms give rise to certain $p$-adic Abel-Jacobi maps at their $p$-new specializations. We intruduce the concept of differentiation of distributions, using it to give a new description of the Coleman-Teitelbaum cocycle that arises in the context of the $\mathcal{L}$-invariant.
\end{abstract}

\section{Contents}

1. Introduction

2. $\mathcal{L}$-invariants of rigid analytic tori and formal logarithms

3. Definite multiplicative integrals and the Manin-Drinfeld Theorem 6

4. Definite additive integrals $\quad 8$

5. Definite additive integrals: proof of the main result 11

6. The analogous results in the indefinite setting $\quad 14$

7. Derivatives of distributions and the Coleman-Teitelbaum cocycle 17

7.1. Generalities on distributions and locally trivial bundles $\quad 17$

7.2. Derivatives of distributions 24

7.3. The relation with the Coleman-Teitelbaum cocycle 28

References $\quad 30$

\section{INTRODUCTION}

After Serre's observations involving the family of Eisentein series (see [29]), a theory of $p$-adic families of modular forms was systematically developed by Hida in the ordinary case (see [23] and [24]), and by Coleman in the more general case of finite slope (see [10]). This theory was subsequently encapsulated geomerically via the eigencurve by Coleman-Mazur (see [11]). Since then, other points of view on $p$-adic families of modular forms have been developed; one of the most flexible is the cohomological approach inspired by the theory of modular symbols and the Eichler-Shimura isomorphism. This approach was developed in a sequence of papers, see [22, 42], with the development culminating in the authoritative [3]. The connection between this cohomological approach and original theories of Coleman and Coleman-Mazur approach to $p$-adic families was spelled out in [2], the essential input being an overconvergent Eichler-Shimura isomorphism.

When the algebraic group underlying the $p$-adic families is the group of units of a definite quaternion algebra, the cohomological approach simplifies, due to the fact that the associated algebraic group is compactmod-center at infinity. In this case, one can define $p$-adic families of modular forms very concretely as in [9]. Such families are similar in spirit to those considered in the first and the third parts of the this paper, while the arguments of $\S 6$ are couched in the language of [3].

There are many ways in which the arithmetic of a modular forms $f$ is determined by the $p$-adic family $f_{\infty}$ passing through it. One such example is proof by Greenberg-Stevens [22] of the Mazur-Tate-Teitelbaum conjecture [27], in which the key point is the identity

$$
\mathcal{L}_{p}\left(A_{f}\right)=-2\left(d \log a_{p}\right)(2) .
$$

The quantity on the left hand side, the $\mathcal{L}$-invariant attached to $f \in S_{2}\left(\Gamma_{0}(N p)\right)^{\text {new }}$, does not involve the family $f_{\infty}$ while the right hand side clearly does. The aim of this paper is to highlight other manifestations

Date: March 8, 2016.

MG's research is supported by NSERC of Canada. 
of this phenomenon, whereby a quantity which depends only on on the modular form $f$ is described by one attached to its deformation $f_{\infty}$. One such manifestation (in fact, a direct analogue of (1)) is Proposition 7.14, relating the Coleman-Teitelbaum cocycle to another cocycle constructed via a differentiation of measures operation described in $\S 7$. Another phenomenon of this kind is obtained showing that the $p$-adic Abel-Jacobi map of an appropriate Shimura curve can be computed by means of the deformations identified with the tangent space to the the Jacobian of the Shimura curve in question. The result is the formula stated in Theorem 1.1; its proof is based on computations of Bertolini, Darmon, and Iovita appearing in [4] and [5]. As an application, one may extend their main result [4, Theorem 5.4] by removing the restriction that the Fourier coefficients of $f$ lie in $\mathbb{Q}$. Our final example is Theorem 6.5, presented in $\S 6$ (see also Theorem 1.1).

Suppose that $p$ is a prime, that $N^{+}$and $N^{-}$are coprime integers, and that $N^{-}$is the squarefree product of an odd number of primes. Fix a complete subfield $E \subset \mathbb{C}_{p}$. We let

$$
\phi \in S_{2}\left(p N^{+}, N^{-}\right)^{p \text {-new }}
$$

be a weight two, $p$-new, cuspidal eigenform on the definite quaternion algebra $B$ over $\mathbb{Q}$ of discriminant $N^{-} \infty$ and level $\Gamma_{0}\left(p N^{+}\right)$. Fix a maximal order $\mathcal{O}_{B} \subset B$ and an Eichler order $R_{0}\left(p N^{+}, N^{-}\right) \subset B$ of level $p N^{+}$. We choose a local isomorphism $\iota_{p}: B_{p} \stackrel{\sim}{\rightarrow} \mathbf{G L}_{2}\left(\mathbb{Q}_{p}\right)$ and let

$$
\widetilde{\Gamma}_{N^{+}, N^{-}}:=\iota_{p}\left(R_{0}\left(p N^{+}, N^{-}\right)[1 / p]^{\times}\right) \subset \mathbf{G L}_{2}\left(\mathbb{Q}_{p}\right)
$$

be the arithmetic group attached to $R_{0}\left(p N^{+}, N^{-}\right)$. Its subgroup of norm one elements $\Gamma_{N^{+}, N^{-}} \subset \widetilde{\Gamma}_{N^{+}, N^{-}}$ is a Schottky group whose associated Mumford curve

$$
\pi_{N^{+}, N^{-}}: \mathcal{H}_{p} \longrightarrow \Gamma_{N^{+}, N^{-}} \backslash \mathcal{H}_{p}
$$

is the rigid analytic space associated to the Shimura curve $X_{N^{+}, N^{-}}$attached to the indefinite quaternion algebra over $\mathbb{Q}$ of discriminant $N^{-} p$; the isomorphism is defined over $\mathbb{Q}_{p^{2}}$, the quadratic unramified extension of $\mathbb{Q}_{p}$ (see $[4, \S 4.1]$ ). Abusing notation slightly, we will frequently identify $\Gamma_{N^{+}, N^{-}} \backslash \mathcal{H}_{p}$ and $X_{N^{+}, N^{-}}$. Write $J_{N^{+}, N^{-}}$for the Jacobian variety of $X_{N^{+}, N^{-} p}$.

We recall that the rigid analytic $p$-adic upper halfplane is a tubular neighborhood of the Bruhat-Tits tree $\mathcal{T}$ whose set of edges (resp. vertices) we denote by $\mathcal{E}$ (resp. $\mathcal{V}$ ). The ends of $\mathcal{T}$ are identified with $\mathbb{P}^{1}\left(\mathbb{Q}_{p}\right)$ and the combinatorics of $\mathcal{T}$ can be used to conveniently describe the $p$-adic topology on $\mathbb{P}^{1}\left(\mathbb{Q}_{p}\right)$ as follows: A basis of compact-open subsets of the topology on the projective line is given by the subsets $U_{e} \subset \mathbb{P}^{1}\left(\mathbb{Q}_{p}\right)$, where $U_{e}$ consists of those points associated to ends of $\mathcal{T}$ emanating from $e$. We refer the reader to [31] for basic notation and facts about the Bruhat-Tits tree. We let $\mathcal{C}_{\text {har }}(\mathcal{E}, E)$ be the space of harmonic cocycles on $\mathcal{T}$, maps $c_{*}: \mathcal{E} \rightarrow E$ such that $c_{\bar{e}}=-c_{e}$ and $\sum_{s(e)=v} c_{e}=0$ for every $v \in \mathcal{V}$.

For a $p$-adic manifold $X$ we write $\mathcal{A}(X)$ be the space of $E$-valued, locally analytic functions on $X$, and $\mathcal{D}(X)$ for its strong $E$-dual (see $\S 7.1)$. Write $\mathcal{A}\left(\mathbb{P}^{1}\left(\mathbb{Q}_{p}\right)\right)^{0} \subset \mathcal{A}\left(\mathbb{Q}_{p}\right)$ for the space of locally analytic functions which are constant at $\infty$ (see $\left[28\right.$, Definition 3.2], for example). We let $\mathcal{D}\left(\mathbb{P}^{1}\left(\mathbb{Q}_{p}\right)\right)^{0}$ be its dual and write $\mathcal{D}^{0}\left(\mathbb{P}^{1}\left(\mathbb{Q}_{p}\right)\right)$ for the subspace of $\mathcal{D}\left(\mathbb{P}^{1}\left(\mathbb{Q}_{p}\right)\right)^{0}$ consisting of those locally analytic distributions $\mu$ such that $\mu(1)=0$.

Because $\phi$ is $p$-new, when $E$ contains the eigenvalues of $\phi$ one can associated to $\phi$ a harmonic cocyle $c_{\phi} \in \mathcal{C}_{\text {har }}(\mathcal{E}, E)^{\Gamma_{N^{+}, N^{-}}}$and a rigid analytic modular form $f_{\phi}$ on $X_{N^{+}, N^{-} p}$ (see [34, (13)]). There is a unique distribution $\mu_{\phi} \in \mathcal{D}^{0}\left(\mathbb{P}^{1}\left(\mathbb{Q}_{p}\right)\right)^{\Gamma_{N^{+}, N^{-}}}$such that $R\left(\mu_{\phi}\right)=c_{\phi}$, where

$$
R: \mathcal{D}^{0}\left(\mathbb{P}^{1}\left(\mathbb{Q}_{p}\right)\right)^{\Gamma_{N^{+}, N^{-}}} \longrightarrow \mathcal{C}_{\text {har }}(\mathcal{E}, E)^{\Gamma_{N^{+}, N^{-}}}
$$

is defined by the rule $R(\mu)_{e}:=\mu\left(\chi_{U_{e}}\right), \chi_{U_{e}}$ being the characteristic function of $U_{e}$.

In order to state our results precisely, we need to introduce a few more spaces of distributions. Setting $W:=\mathbb{Q}_{p}^{2}-\{0\}$ defines a trivial $\mathbb{Q}_{p}^{\times}$-bundle

$$
\pi: W \longrightarrow \mathbb{P}^{1}\left(\mathbb{Q}_{p}\right), \quad \pi(x, y):=[x: y] .
$$

Let $\mathcal{O}$ be an affinoid $E$-algebra and let $\omega: \mathbb{Z}_{p}^{\times} \rightarrow \mathcal{O}^{\times}$be a continuous character; it extends uniquely to a homomorphism of algebras $\omega: \mathcal{D}\left(\mathbb{Z}_{p}^{\times}\right) \rightarrow \mathcal{O}$. Write $\mathcal{A}_{0}(W)$ for the space of $E$-valued locally analytic 
functions on $W$ such that $F(p x, p y)=F(x, y)$ and let $\mathcal{D}_{0}(W)$ be its strong dual. Then $\mathcal{D}_{0}(W)$ is naturally a $\mathcal{D}\left(\mathbb{Z}_{p}^{\times}\right)$-module and, regarding $\mathcal{O}$ as a $\mathcal{D}\left(\mathbb{Z}_{p}^{\times}\right)$-module via $\omega$, we may form the completed tensor product

$$
\mathcal{D}_{\omega, 0}(W):=\mathcal{O} \widehat{\otimes}_{\omega} \mathcal{D}_{0}(W) .
$$

If $\varphi: \mathcal{O} \rightarrow \mathcal{O}^{\prime}$ is a morphism of $E$-algebras then there is a natural specialization map

$$
\mathcal{D}_{\omega, 0}(W) \longrightarrow \mathcal{D}_{\omega^{\prime}, 0}(W), \quad \omega^{\prime}:=\varphi \circ \omega .
$$

(The reader may consult (61) for the precise definition of this map). Recall that continuous characters from $\mathbb{Z}_{p}^{\times}$are parametrized by the weight space $\mathcal{X}:=\operatorname{Hom}_{c t s}\left(\mathbb{Z}_{p}^{\times}, \mathbf{G}_{m}\right)$. Suppose that $\varphi: \mathcal{O}(\Omega) \rightarrow E$ is the morphism corresponding to $0 \in \Omega$, for an open affinoid $\Omega \subset \mathcal{X}$ containing the weight $0:=\left[t \mapsto t^{0}=1\right]$. The inclusion $\Omega \subset \mathcal{X}$ corresponds to a character $\omega: \mathbb{Z}_{p}^{\times} \rightarrow \mathcal{O}^{\times}$and, in this case, we write $\mathcal{D}_{\Omega, 0}(W):=\mathcal{D}_{\omega, 0}(W)$; we can also consider $\mathcal{D}_{0,0}(W)=\mathcal{D}_{\omega^{\prime}, 0}(W)$ with $\omega^{\prime}=0$. With this notation, (3) can be written in the form

$$
\mathcal{D}_{\Omega, 0}(W) \longrightarrow \mathcal{D}_{0,0}(W) \text {. }
$$

The invariance property of $F \in \mathcal{A}_{0}(W)$ implies that $F$ is uniquely determined by its restriction to $L_{*}^{\prime}:=$ $\mathbb{Z}_{p}^{2}-p \mathbb{Z}_{p}^{2}$, and one easily checks thatthere is a canonical isomorphism $\mathcal{D}\left(\mathbb{P}^{1}\left(\mathbb{Q}_{p}\right)\right)^{0}=\mathcal{D}_{0,0}(W)$ (see $[21$, proof of Lemma 6.2] and [35, Remark 5]). We use this identification to vary $\mu_{\phi}$ in a $p$-adic family.

Let $\mathcal{C}\left(\mathcal{V}, \mathcal{D}_{\Omega, 0}(W)\right)^{\widetilde{\Gamma}_{N^{+}, N^{-}}}$be the space of $\widetilde{\Gamma}_{N^{+}, N^{-}}$-invariant maps $c: \mathcal{V} \rightarrow \mathcal{D}_{\Omega, 0}(W)$ (see [34, Proposition $3.5]$ for a description in terms of lattices). We write $\mathcal{V}^{+}$for the set of vertices at even distance from $v_{*}=\left[L_{*}\right]$. Then there exists an eigenfamily of distributions $\mu_{\Omega, *}:=\left\{\mu_{\Omega, v}\right\}_{v \in \mathcal{V}}$ with the property that, writing $\mu_{0, v}$ for the specialization of $\mu_{\Omega, v}$ at 0 obtained via (4), we have

$$
\mu_{0, v}=\mu_{\phi} \text { for every } v \in \mathcal{V}^{+} .
$$

(See [4, Theorem 2.5 and Lemma 2.12] and [34, Theorem 3.7 and subsequent discussion, Proposition 3.8]).

For an element $\tau \in \mathcal{H}_{p}^{\text {ur }}:=\mathbb{Q}_{p}^{\text {ur }}-\mathbb{Q}_{p}$, we write $r(\tau) \in \mathcal{V}$ for its reduction (see [13, Proposition 5.1]) and consider the quantity

$$
\frac{d}{d \kappa}\left[\mu_{\Omega, r(\tau)}\langle x-\tau y\rangle^{\kappa}\right]_{\kappa=0} \in E .
$$

It can be shown that it does not depend on the choice of family $\mu_{\Omega, *}$ satisfying (5); the proof is similar to that of [21, Lemma 7.1].

The Picard variety $J_{N^{+}, N^{-}}$is endowed with a canonical morphism

$$
\operatorname{AJ}_{X_{N^{+}, N^{-}}}^{0}:\left(\operatorname{Div}^{0} X_{N^{+}, N^{-}}\right)(E):=\left(\operatorname{Div}^{0} X_{N^{+}, N^{-} p}\left(\mathbb{C}_{p}\right)\right)^{G_{\Phi_{p} / E}} \longrightarrow J_{N^{+}, N^{-} p}(E) .
$$

(We write $G_{K^{\prime} / K}$ for the associated Galois group of $K^{\prime} / K$ and $G_{K}$ for $G_{\bar{K} / K}$.) For every $q \nmid p N$, the Hecke operator $t_{q}$ has degree $q+1$ and it follows that, setting $t_{q}:=(q+1)-T_{q}$, we have

$$
t_{q}: \operatorname{Div} X_{N^{+}, N^{-} p} \longrightarrow \operatorname{Div}^{0} X_{N^{+}, N^{-} p} \text {. }
$$

Let $T_{0}\left(J_{N^{+}, N^{-}}\right)$be the tangent space to $J_{N^{+}, N^{-}}$at the identity, whose $E$-points are identified with (apply $[6$, Ch. $8, \S 8.4$, Theorem 1] and Serre duality):

$$
T_{0}\left(J_{N^{+}, N^{-} p}\right)(E)=\operatorname{Hom}_{E}\left(H^{0}\left(X_{N^{+}, N^{-} p / E}, \Omega_{X_{N^{+}, N^{-} p / E}^{1}}^{1}\right), E\right) .
$$

The formal logarithm is a map

$$
\log _{J_{N^{+}, N^{-}}}: J_{N^{+}, N^{-} p}(E) \longrightarrow T_{0}\left(J_{N^{+}, N^{-}}\right)(E) .
$$

Noticing that $t_{q}$ is invertible on $T_{0}\left(J_{N^{+}, N^{-}}\right)$we may define the following composite:

$$
\log _{J_{N^{+}, N^{-}}} \mathrm{AJ}:=t_{q}^{-1} \circ \log _{J_{N^{+}, N^{-}}} \circ \mathrm{AJ}_{X_{N^{+}, N^{-}}}^{0} \circ t_{q}: \operatorname{Div} X_{N^{+}, N^{-} p} \longrightarrow T_{0}\left(J_{N^{+}, N^{-} p}\right) \text {. }
$$

We remark that this definition is indeed independent of $q \nmid p N$.

We recall that taking residues of the pull-back to $\mathcal{H}_{p}$ of a differential form $\omega \in H^{0}\left(X_{N^{+}, N^{-} p, E}, \Omega_{X_{N^{+}, N^{-} p, E}^{1}}^{1}\right)$ yields an identification

$$
H^{0}\left(X_{N^{+}, N^{-} p, E}, \Omega_{X_{N^{+}, N^{-}, E}^{1}}^{1}\right) \stackrel{\sim}{\longrightarrow} \mathcal{C}_{\text {har }}(\mathcal{E}, E)^{\Gamma_{N^{+}, N^{-}}}
$$


Let $\omega_{\phi}$ be the differential form whose residues are given by $c_{\phi}$. Changing our point of view slightly, we assume that $\omega \in H^{0}\left(X_{N^{+}, N^{-} p, E}, \Omega_{X_{N^{+}, N^{-}, E}}^{1}\right)$ gives rise to $c_{\omega} \in \mathcal{C}_{\text {har }}(\mathcal{E}, E)^{\Gamma_{N^{+}, N^{-}}}$which, in turn, gives a measure $\mu_{\omega} \in \mathcal{D}^{0}\left(\mathbb{P}^{1}\left(\mathbb{Q}_{p}\right)\right)^{\Gamma_{N^{+}, N^{-}}}$. We choose a family $\mu_{\Omega, *}^{\omega}$ such that $\mu_{0, v}^{\omega}=\mu_{\omega}$ for every $v \in \mathcal{V}^{+}$.

The main result of the first part of this paper is the following:

Theorem 1.1. Let $\tau \in \mathcal{H}_{p}^{\mathrm{ur}}$. Writing $[\tau]$ for the natural image of $\tau$ in $\left(\operatorname{Div} X_{N^{+}, N^{-}}\right)\left(\mathbb{Q}_{p}^{\mathrm{ur}}\right)$, we have

$$
\left(\log _{J_{N^{+}, N^{-}}} A J\right)([\tau])(\omega)=\frac{d}{d \kappa}\left[\mu_{\Omega, r(\tau)}^{\omega}\langle x-\tau y\rangle^{\kappa}\right]_{\kappa=0} .
$$

The first part of the paper is devoted to the proof of this result. Theorem 1.1, specialized to the quotient $J_{N^{+}, N^{-} p} \rightarrow E_{f}$ of $J_{N^{+}, N^{-}}$associated to a weight 2 modular form with rational Fourier coefficients, is a key ingredient in the proof of the main result of [4]. Our results can be viewed as a lift to the Jacobian of the computations appearing there, yielding generalizations of the corresponding theorems.

In $\S 6$ we move to the setting where $N^{-}$is a squarefree product of an even number of primes. Here, we first lift the original construction of [12], as generalized in [19]. Our approach is a bit different from related constructions of of [15] and [26], but the resulting rigid analytic varieties are isogenous. Then we prove the analogue of Theorem 1.1 in Theorem 6.5. As an application, we can lift the rationality main result of [25] on rationality of Darmon points to the Jacobians. This result can also be deduced from the rationality result of [21], specialized to weight 2 modular forms, using $\S 6$ to translate the results of loc. cit. to our setting (see [21, Remarks 1.11 and $\S 1.4])$.

Finally, $\S 7$ introduces the notion of derivative of a family of distributions in complete generality. This is employed in order to "differentiate" the family specializing to $\mu_{\phi}$ (possibly of higher, even weight) and attach to it a cocycle in $H^{1}\left(\Gamma_{N^{+}, N^{-}}, \mathbf{V}_{k}\right)$. As an application, in Proposition 7.14 we get a new description of the Coleman-Teitelbaum cocycle in terms of differentiation of families of distributions. In particular, an a priori a new definition of the Teitelbaum $\mathcal{L}$-invariant can be proposed and, possibly, generalized to other settings. Let us remark that, the formula which appears in Proposition 7.14 is another manifestation of the phenomenon relating invariants of modular forms (the Coleman cocycle) to quantities defined using $p$-adic deformations (the derivative of the family passing through it). It is, thus, of the same nature as (1) and the formulas appearing in Theorem 1.1 and Theorem 6.5. Because $p$-adic $L$-functions are defined in terms of measures, we hope to apply these notions to the study of derivatives of $p$-adic $L$-functions in other settings.

Regarding notation for the various arithmetic group actions appearing in this paper, we follow the convention of [28].

\section{2. $\mathcal{L}$-INVARIANTS OF RIGID ANALYTIC TORI AND FORMAL LOGARITHMS}

In the literature, $\mathcal{L}$-invariants have been attached to a variaty of objects, e.g., abelian varieties, $p$-adic representations, and modular forms. The aim of this section to review the definition of the $\mathcal{L}$-invariant $\mathcal{L}(A)$ (see $[22, \S 3,(3.5)$ definition]) attached to an abelian variaty, to extend this definition to rigid analytic tori, and to relate this $\mathcal{L}$-invariant to the formal logarithm map.

We work over our complete field $E \subset \mathbb{C}_{p}$, assumed to be locally compact in this section. We write $k_{E}$ for the residue field of $E$. From now on we will write $\log _{\lambda}: E^{\times} \rightarrow E$ for the choice of the $p$-adic $\operatorname{logarithm}$ determined by $\log _{\lambda}(p)=-\lambda$, where $\lambda \in E$; hence $\log _{0}=\log (\langle\cdot\rangle)$ and $\log _{\lambda}=\log _{0}-\lambda$ ord. Recall that, if $A=A_{/ E}$ is as above, then the dual abelian variety $B$ has the same reduction type and, hence, they both admit a $p$-adic uniformization (see for example $[37, \S 1]$ for a short account on $p$-adic uniformization). Indeed, there are canonical free $\mathbb{Z}$-modules $X$ and $Y$ of $\operatorname{rank} \operatorname{dim}(A)$ (the characters groups of $B_{/ k_{E}}^{0}$ and, respectively, $A_{/ k_{E}}^{0}$ ) on which $G_{E}$ acts trivially, a $\mathbb{Z}$-linear pairing

$$
j: X \otimes_{\mathbb{Z}} Y \longrightarrow E^{\times}
$$

and exact $G_{E^{\prime} / E^{-} \text {-equivariant sequences }}$

$$
\begin{gathered}
0 \longrightarrow X \stackrel{j}{\longrightarrow} \operatorname{Hom}\left(Y, E^{\prime \times}\right) \longrightarrow A\left(E^{\prime}\right) \longrightarrow 0, \\
0 \longrightarrow Y \stackrel{j}{\longrightarrow} \operatorname{Hom}\left(X, E^{\prime \times}\right) \longrightarrow B\left(E^{\prime}\right) \longrightarrow 0 \\
4
\end{gathered}
$$


for every $E \subset E^{\prime} \subset \bar{E}$.

A continuous group homomorphism $\ell: E^{\times} \rightarrow E$ induces a map $\ell: \operatorname{Hom}\left(Y, E^{\times}\right) \rightarrow \operatorname{Hom}(Y, E)$. Taking $E^{\prime}=E$, we define:

$$
j_{\ell}:=\ell \circ j: X \otimes_{\mathbb{Z}} Y \longrightarrow E \quad \operatorname{and}_{\ell}:=\ell \circ j: X \longrightarrow \operatorname{Hom}(Y, E)=: T_{0}(A)(E) .
$$

Set $X_{E}:=E \otimes_{\mathbb{Z}} X$ and $Y_{E}:=E \otimes_{\mathbb{Z}} Y$ and write $j_{\ell, E}$ for the corresponding maps

$$
j_{\ell, E}: X_{E} \otimes_{E} Y_{E} \longrightarrow E \quad \text { and } \quad j_{\ell, E}: X_{E} \rightarrow \operatorname{Hom}\left(Y_{E}, E\right)=T_{0}(A)(E) .
$$

Noticing that $j_{\text {ord }}$ is non-degenerate, the $\mathcal{L}$-invariant $\mathcal{L}(A)$ is defined in [22, $\S 3,(3.5)$ definition] to be the unique $E$-linear endomorphism $\mathcal{L}(A): X_{E} \rightarrow X_{E}$ such that

$$
j_{\log _{0}, E}(x, y)=j_{\text {ord }, E}(\mathcal{L}(A) x, y) .
$$

On the other hand, since $j_{\text {ord, } E}$ is non-degenerate, it induces an isomorphism $j_{\text {ord, } E}: X_{E} \stackrel{\sim}{\rightarrow} T_{0}(A)(E)$ and there is a unique $E$-linear endomorphism $\mathcal{L}^{\prime}(A): T_{0}(A)(E) \rightarrow T_{0}(A)(E)$ such that

$$
j_{\log _{0}, E}=\mathcal{L}^{\prime}(A) \circ j_{\text {ord }, E}: X_{E} \longrightarrow T_{0}(A)(E)
$$

If we transport $\mathcal{L}(A)$ to $X_{E}$ via $j_{\text {ord, } E}$, it is easy to see that $(9)$ and $(10)$ are equivalent, i.e. $\mathcal{L}^{\prime}(A)=\mathcal{L}(A)$. Furthermore, since $X_{E}:=E \otimes_{\mathbb{Z}} X,(10)$ is equivalent to

$$
j_{\log _{0}}=\mathcal{L}^{\prime}(A) \circ j_{\text {ord }}: X \rightarrow T_{0}(A)(E) .
$$

We now assume that we are given a rigid analytic torus $J=J_{/ E}$ defined by an exact sequence of the form

$$
0 \longrightarrow X \stackrel{j}{\longrightarrow} \operatorname{Hom}\left(Y, \mathbf{G}_{\mathrm{m}}^{\mathrm{an}}\right) \longrightarrow J \rightarrow 0
$$

where $X$ and $Y$ are free $\mathbb{Z}$-modules of the same rank on which $G_{E}$ acts trivially By the definition of a rigid analytic torus, $j$ factors through

$$
j: X \longrightarrow \operatorname{Hom}\left(Y, E^{\times}\right),
$$

identifying $X$ with a lattice in $\operatorname{Hom}\left(Y, E^{\times}\right)$that is discrete in $\operatorname{Hom}\left(Y, \mathbf{G}_{\mathrm{m}}^{\text {an }}\right)$, i.e. such that its intersection with every affinoid is finite (see [36, §4.2]). The underlying $E$-analytic manifold of $J$ is

$$
J(E):=\frac{\operatorname{Hom}\left(Y, E^{\times}\right)}{X}
$$

and the tangent space to $J$ and $J(E)$ at the identity are identified with $T_{0}(J)(E):=\operatorname{Hom}(Y, E)$ (and agree with $T_{0}(A)(E)$ when $J=A^{a n}$ is the rigid analytic manifold attached to an abelian variety $A$ over $E$ ). Let us write

$$
\log _{J}: J(E) \longrightarrow T_{0}(J)(E)
$$

for the formal logarithm as defined, for example, in [7, Ch. III, §7, no. 6, Def. 2, Prop. 10 and Prop. 13]. We can define

$$
j_{\ell}:=\ell \circ j: X \longrightarrow T_{0}(J)(E)
$$

using (12) in this lightly more general setting. We take (11) as the definition of the $\mathcal{L}$-invariant $\mathcal{L}(J)$ of $J$, i.e. $\mathcal{L}(J): T_{0}(J)(E) \rightarrow T_{0}(J)(E)$ is the unique $E$-linear endomorphism such that

$$
j_{\log _{0}}=\mathcal{L}(J) \circ j_{\text {ord }}: X \longrightarrow T_{0}(J)(E) .
$$

The following simple result connects $\mathcal{L}$-invariants and formal logarithms.

Lemma 2.1. The formal logarithm is explicitly given by the rule

$$
\log _{J}([z])=\log _{0} \circ z-\mathcal{L}(J)(\text { ord } \circ z),
$$

where $z \in \operatorname{Hom}\left(Y, E^{\times}\right)$represents $[z] \in J(E)$.

Proof. The formal logarithm is characterized as the unique group homomorphism $J(E) \longrightarrow T_{0}(J)(E)$ that is locally analytic at the identity with differential the identity (see [7, Ch. III, §7, no. 6, Prop. 10]). Since $\log _{0}$ has this property and the differential of ord is zero, we can conclude that the differential of the right hand side of (14) has this property once we have checked that it is well defined on the quotient $J$. By definition of $j_{\ell}(x)=\ell \circ j(x)$, we see that, for every $x \in X$,

$$
\log _{0} \circ j(x)-\mathcal{L}(\operatorname{ord} \circ j(x))=\underset{5}{j_{\log _{0}}}(x)-\mathcal{L}(J)\left(j_{\text {ord }}(x)\right) \stackrel{(13)}{=} 0 .
$$




\section{Definite multiplicative integrals and the Manin-Drinfeld Theorem}

Let $C\left(\mathbb{P}^{1}\left(\mathbb{Q}_{p}\right), E^{\times}\right)$be the space of continuous, $E^{\times}$-valued functions and let $\mathcal{C}_{\text {har }}(\mathcal{E}, \mathbb{Z})$ be the space of $\mathbb{Z}$-valued harmonic cocyles. We can define a $\mathbf{G L}_{2}\left(\mathbb{Q}_{p}\right)$-equivariant pairing

$$
(-,-): \mathcal{C}_{\text {har }}(\mathcal{E}, \mathbb{Z}) \otimes_{\mathbb{Z}} C\left(\mathbb{P}^{1}\left(\mathbb{Q}_{p}\right), E^{\times}\right) / E^{\times} \longrightarrow E^{\times}
$$

induced by the rule (cf. $[19, \S 9]$ and $[4,(63)])$

$$
(c, f):=\lim _{\mathcal{T}_{0} \subset \mathcal{T}} \prod_{e \in \partial \mathcal{T}_{0}} f\left(t_{e, \mathcal{T}_{0}}\right)^{c(e)}, \quad f \in C\left(\mathbb{P}^{1}\left(\mathbb{Q}_{p}\right), E^{\times}\right) .
$$

Here, $\mathcal{T}_{0}$ runs over all the net of finite connected subtrees of $\mathcal{T}, \partial \mathcal{T}_{0}$ denotes the boundary edges of $\mathcal{T}_{0}$ oriented towards $\partial \mathcal{T}=\mathbb{P}^{1}\left(\mathbb{Q}_{p}\right)$ and $t_{e, \mathcal{T}_{0}}$ is an arbitrary point in $U_{e}$. The limit exists, it is well defined and induces (15).

For every $\tau_{1}, \tau_{2} \in \mathcal{H}_{p}$, let us consider the function

$$
\theta_{\times}^{\tau_{2}-\tau_{1}}(z):=\frac{z-\tau_{2}}{z-\tau_{1}}, \quad z \in \mathbb{P}^{1}\left(\mathbb{Q}_{p}\right) .
$$

Write $\left[\theta_{\times}^{\tau_{2}-\tau_{1}}\right]$ for its image in $C\left(\mathbb{P}^{1}\left(\mathbb{Q}_{p}\right), E^{\times}\right) / E^{\times}$. Define

$$
\Delta(E)=\left(\operatorname{Div} \mathcal{H}_{p}\right)^{G_{\bar{\Phi}_{p} / E}} \quad \text { and } \quad \Delta^{0}(E)=\left(\operatorname{Div}^{0} \mathcal{H}_{p}\right)^{G_{\bar{\Phi}_{p} / E}}
$$

Then we have the following exact sequence:

$$
0 \rightarrow \Delta^{0}(E) \stackrel{i}{\longrightarrow} \Delta(E) \stackrel{\text { deg }}{\longrightarrow} E \longrightarrow 0 .
$$

Define a $\mathbf{G L}_{2}\left(\mathbb{Q}_{p}\right)$-equivariant integration pairing

$$
I_{\times}^{0}: \mathcal{C}_{\text {har }}(\mathcal{E}, \mathbb{Z}) \otimes_{\mathbb{Z}} \Delta^{0}(E) \rightarrow E^{\times}
$$

by linear extension of the rule $I_{\times}^{0}\left(c, \tau_{2}-\tau_{1}\right):=\left(c,\left[\theta_{\times}^{\tau_{2}-\tau_{1}}\right]\right)$.

The integration pairing $I_{\times}^{0}$ gives rise to a morphism

$$
I_{\times}^{0}:\left(\Delta^{0}(E)\right)_{\Gamma_{N^{+}, N^{-}}} \longrightarrow \operatorname{Hom}\left(\mathcal{C}_{\text {har }}(\mathcal{E}, \mathbb{Z})^{\Gamma_{N^{+}, N^{-}}, E^{\times}}\right)=: \mathbf{T}_{\Gamma_{N^{+}, N^{-}}}(E) .
$$

On the other hand (17) induces the exact sequence

$$
H_{1}\left(\Gamma_{N^{+}, N^{-}}, \mathbb{Z}\right) \stackrel{\partial}{\longrightarrow}\left(\Delta^{0}(E)\right)_{\Gamma_{N^{+}, N^{-}}} \stackrel{i}{\longrightarrow}(\Delta(E))_{\Gamma_{N^{+}, N^{-}}} \stackrel{\text { deg }}{\rightarrow} \mathbb{Z} \longrightarrow 0
$$

Defining $\mathbf{q}_{\Gamma_{N^{+}, N^{-}}}:=\operatorname{Im}\left(I_{\times}^{0} \circ \partial\right)$, it follows from that $I_{\times}^{0}$ induces

$$
\mathrm{AJ}^{0}=\mathrm{AJ}_{\Gamma_{N^{+}, N^{-}}}^{0}:\left(\Delta^{0}(E)\right)_{\Gamma_{N^{+}, N^{-}}} \longrightarrow \frac{\mathbf{T}_{\Gamma_{N^{+}, N^{-}}}(E)}{\mathbf{q}_{\Gamma_{N^{+}, N^{-}}}}=: J_{\Gamma_{N^{+}, N^{-}}}(E) .
$$

Proposition 3.1. We have that $\mathbf{q}_{\Gamma_{N^{+}, N^{-}}} \subset \mathbf{T}_{\Gamma_{N^{+}, N^{-}}}\left(\mathbb{Q}_{p}\right)$ is Hecke stable and the quotient $J_{\Gamma_{N^{+}, N^{-}}}(E)$ is represented by a rigid analytic torus $J_{\Gamma_{N^{+}, N^{-}}}$over $\mathbb{Q}_{p}$ endowed with an action of the Hecke algebra.

Proof. The fact that $\mathbf{q}_{\Gamma_{N^{+}, N^{-}}}$is Hecke stable follows from the fact that $\partial$ and $I_{\times}^{0}$ are Hecke equivariant. The proof of the other assertions will be given after Lemma 4.1.

Let

$$
\mathrm{AJ}_{X_{N^{+}, N^{-}}}^{0}: \operatorname{Div}^{0} X_{N^{+}, N^{-} p} \longrightarrow J_{N^{+}, N^{-} p}
$$

be the algebraic Abel-Jacobi map. 
Theorem 3.2. (Manin-Drinfeld) There is a unique isomorphism $J_{\Gamma_{N^{+}, N^{-}}} \rightarrow J_{N^{+}, N^{-} p}$ over $\mathbb{Q}_{p^{2}}$ making the following diagram commutative for every complete field $E \subset \mathbb{C}_{p}$ :

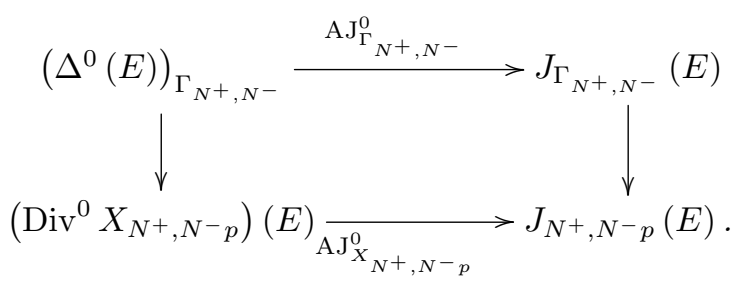

This isomorphism is Hecke equivariant.

Proof. We base our computations on those of [14, $\$ 2.2$ and $\S 2.3]$ that we briefly recall. Let us write $\mathcal{T}_{\Gamma_{N^{+}, N^{-}}}:=\Gamma_{N^{+}, N^{-}} \backslash \mathcal{T}$ for the quotient graph, whose oriented edges are $\mathcal{E}_{\Gamma_{N^{+}, N^{-}}}:=\Gamma_{N^{+}, N^{-}} \backslash \mathcal{E}$ and whose vertices are $\mathcal{V}_{\Gamma_{N^{+}, N^{-}}}:=\Gamma_{N^{+}, N^{-}} \backslash \mathcal{V}$ (Note that the action of $\Gamma_{N^{+}, N^{-}}$is orientation preserving.) Define $\overline{\operatorname{Div}}\left(\mathcal{E}_{\Gamma_{N^{+}, N^{-}}}\right)$to be the quotient of $\operatorname{Div}\left(\mathcal{E}_{\Gamma_{N^{+}, N^{-}}}\right)$by the relations $e+\bar{e}=0$ for every $e \in \mathcal{E}_{\Gamma_{N^{+}, N^{-}}}$. Sending a vertex $v$ to $\delta_{s}(v):=\sum_{s(e)=v} e$ induces an injective map

$$
0 \longrightarrow \operatorname{Div}\left(\mathcal{V}_{\Gamma_{N^{+}, N^{-}}}\right) \stackrel{\delta_{\Im}}{\rightarrow} \overline{\operatorname{Div}}\left(\mathcal{E}_{\Gamma_{N^{+}, N^{-}}}\right) \longrightarrow \operatorname{Div}^{\text {har }}\left(\mathcal{E}_{\Gamma_{N^{+}, N^{-}}}\right) \rightarrow 0
$$

where Div ${ }^{\text {har }}\left(\mathcal{E}_{\Gamma_{N^{+}, N^{-}}}\right)$is, by definition, the cokernel of $\delta_{s}$. It follows that

$$
\mathcal{C}_{\text {har }}(\mathcal{E}, \mathbb{Z})^{\Gamma_{N^{+}, N^{-}}}=\operatorname{Hom}\left(\operatorname{Div}^{\text {har }}\left(\mathcal{E}_{\Gamma_{N^{+}, N^{-}}}\right), \mathbb{Z}\right) .
$$

Consider the canonical morphism

$$
\operatorname{Div}^{\text {har }}\left(\mathcal{E}_{\Gamma_{N^{+}, N^{-}}}\right) \longrightarrow\left(\operatorname{Div}^{\text {har }}\left(\mathcal{E}_{\Gamma_{N^{+}, N^{-}}}\right)\right)^{\vee \vee}=\left(\mathcal{C}_{\text {har }}(\mathcal{E}, \mathbb{Z})^{\Gamma_{N^{+}, N^{-}}}\right)^{\vee}
$$

where we write $X^{\vee}:=\operatorname{Hom}(X, \mathbb{Z})$. By abstract nonsense, $(22)$ is canonically the torsion free quotient of $\operatorname{Div}^{\text {har }}\left(\mathcal{E}_{\Gamma_{N^{+}, N^{-}}}\right)$. As explained in $[14, \S 2.3]$, it is the universal module considered in $[14, \S 2.2]$ in case $\mathcal{M}=\mathbb{Z}$, and $[14,(2.6)]$ becomes

$$
I_{\times}^{0}:\left(\Delta^{0}(E)\right)_{\Gamma_{N^{+}, N^{-}}} \longrightarrow\left(\mathcal{C}_{\text {har }}(\mathcal{E}, \mathbb{Z})^{\Gamma_{N^{+}, N^{-}}}\right)^{\vee} \otimes E^{\times}=\operatorname{Hom}\left(\mathcal{C}_{\text {har }}(\mathcal{E}, \mathbb{Z})^{\Gamma_{N^{+}, N^{-}}, E^{\times}}\right)
$$

Our notation is justified by the fact that $I_{\times}^{0}$ is indeed explicitly given by the morphism (18).

On the other hand, the mapping $e \mapsto \partial(e):=t(e)-s(e)$ induces an exact sequence

$$
0 \longrightarrow \operatorname{Div}_{\text {har }}\left(\mathcal{E}_{\Gamma_{N^{+}, N^{-}}}\right) \longrightarrow \overline{\operatorname{Div}}\left(\mathcal{E}_{\Gamma_{N^{+}, N^{-}}}\right) \stackrel{\partial}{\longrightarrow} \operatorname{Div}^{0}\left(\mathcal{V}_{\Gamma_{N^{+}, N^{-}}}\right) \rightarrow 0
$$

where $\operatorname{Div}_{\text {har }}\left(\mathcal{E}_{\Gamma_{N^{+}, N^{-}}}\right)$is defined to be the kernel. As explained in [14, §2.3], there is a natural pairing

$$
(-,-): \overline{\operatorname{Div}}\left(\mathcal{E}_{\Gamma_{N^{+}, N^{-}}}\right) \otimes_{\mathbb{Z}} \overline{\operatorname{Div}}\left(\mathcal{E}_{\Gamma_{N^{+}, N^{-}}}\right) \longrightarrow \mathbb{Z}
$$

induced by the pairing on Div $\left(\mathcal{E}_{\Gamma_{N^{+}, N^{-}}}\right)$defined by declaring that $e$ and $e^{\prime}$ are orthogonal when $e^{\prime} \neq e$ or $\bar{e}$, while $(e, e)=(e, \bar{e})=-1$. As is also observed in loc. cit., $e \mapsto(e,-)$ induces an identification

$$
\operatorname{Div}^{\text {har }}\left(\mathcal{E}_{\Gamma_{N^{+}, N^{-}}}\right)_{\text {free }} \stackrel{\sim}{\longrightarrow} \operatorname{Hom}\left(\operatorname{Div}_{\text {har }}\left(\mathcal{E}_{\Gamma_{N^{+}, N^{-}}}\right), \mathbb{Z}\right)
$$

where Div ${ }^{\text {har }}\left(\mathcal{E}_{\Gamma_{N^{+}, N^{-}}}\right)$free is the torsion free quotient of Div ${ }^{\text {har }}\left(\mathcal{E}_{\Gamma_{N^{+}, N^{-}}}\right)$. There are natural spectral sequences in $\Gamma_{N^{+}, N^{-}}$-equivariant homology, which degenerate to a long exact sequence from which we extract the following exact sequence (see [8, Ch. VII, $\S 7,(7.3)$ and (7.7)] and [31, Ch II, $\S 2.8$, Proposition 13]):

$$
\bigoplus_{\sigma \in \mathcal{V}_{\Gamma_{N^{+}, N^{-}}}} H_{1}\left(\Gamma_{N^{+}, N^{-}}^{v}, \mathbb{Z}\right) \longrightarrow H_{1}\left(\Gamma_{N^{+}, N^{-}}, \mathbb{Z}\right) \rightarrow \operatorname{Div}_{\text {har }}\left(\mathcal{E}_{\Gamma_{N^{+}, N^{-}}}\right) \longrightarrow 0 .
$$


Here, $\Gamma_{N^{+}, N^{-}}^{v} \subset \Gamma_{N^{+}, N^{-}}$is the (finite) stabilizer of $v$ in $\Gamma_{N^{+}, N^{-}}$and $H_{1}\left(\Gamma_{N^{+}, N^{-}}, \mathbb{Z}\right)=\Gamma_{N^{+}, N^{-}}^{\text {ab }}$, canonically. It follows that the left-most term of (24) is finite (see [30, Ch. VIII, §2, Corollaries 1 and 2]), implying that the epimorphism in (24) gives an isomorphism

$$
\operatorname{Hom}\left(\operatorname{Div}_{\text {har }}\left(\mathcal{E}_{\Gamma_{N^{+}, N^{-}}}\right), \mathbb{Z}\right) \stackrel{\sim}{\longrightarrow} \operatorname{Hom}\left(\Gamma_{N^{+}, N^{-}}^{a b}, \mathbb{Z}\right) .
$$

Applying $(-) \otimes_{\mathbb{Z}} E^{\times}$and employing the canonical identification $X^{\vee} \otimes_{\mathbb{Z}} E^{\times}=\operatorname{Hom}\left(X, E^{\times}\right)$, we see that $(25) \circ(23) \circ(22)_{\text {free }}$ gives

$$
\begin{aligned}
\lambda & : \operatorname{Hom}\left(\mathcal{C}_{\text {har }}(\mathcal{E}, \mathbb{Z})^{\Gamma_{N^{+}, N^{-}}}, E^{\times}\right)=\left(\mathcal{C}_{\text {har }}(\mathcal{E}, \mathbb{Z})^{\Gamma_{N^{+}, N^{-}}}\right)^{\vee} \otimes E^{\times} \\
& =\operatorname{Div}^{\text {har }}\left(\mathcal{E}_{\Gamma_{N^{+}, N^{-}}}\right)_{\text {free }} \otimes_{\mathbb{Z}} E^{\times} \stackrel{\sim}{\longrightarrow}\left(\Gamma_{N^{+}, N^{-}}^{a b}\right)^{\vee} \otimes_{\mathbb{Z}} E^{\times}=\operatorname{Hom}\left(\Gamma_{N^{+}, N^{-}}^{a b}, E^{\times}\right)
\end{aligned}
$$

We deduce that there is a commutative diagram

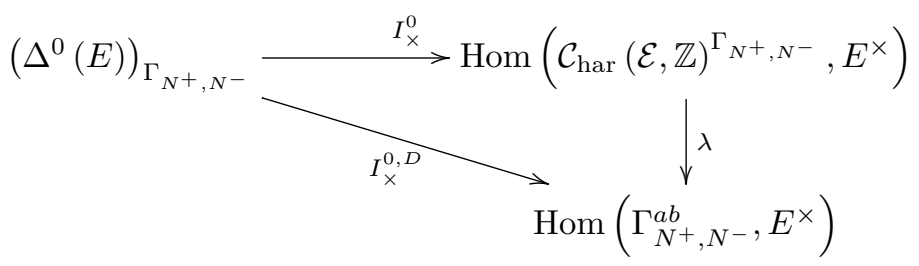

where $I_{\times}^{0, D}$ is the left hand side of $[14,(2.13)]$, as the reader may check using its explicit expression [14, (2.14)]. Let us set $\mathbf{q}_{\Gamma_{N^{+}, N^{-}}}^{D}:=\operatorname{Im}\left(I_{\times}^{0, D} \circ \partial\right)$ so that

$$
J_{\Gamma_{N^{+}, N^{-}}}^{D}(E):=\frac{\operatorname{Hom}\left(\Gamma_{N^{+}, N^{-}}^{a b}, E^{\times}\right)}{\mathbf{q}_{\Gamma_{N^{+}, N^{-}}}^{D}}
$$

is the rigid analytic torus considered in [14]. Writing

$$
\mathrm{AJ}^{0, D}:\left(\Delta^{0}(E)\right)_{\Gamma_{N^{+}, N^{-}}} \longrightarrow \frac{\operatorname{Hom}\left(\Gamma_{N^{+}, N^{-}}^{a b}, E^{\times}\right)}{\mathbf{q}_{\Gamma_{N^{+}, N^{-}}}^{D}}=: J_{\Gamma_{N^{+}, N^{-}}}^{D}(E)
$$

for the induced morphism, (26) yields the following commutative diagram:

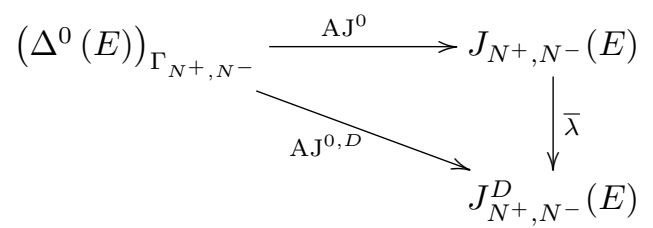

Thanks to (27), we see that $\bar{\lambda}$ identifies our analytic torus with that of [14] and our $\mathrm{AJ}^{0}$ corresponds to the map $\mathrm{AJ}^{0, D}$ induced by the multiplicative integral $I_{\times}^{0, D}$, the left hand side of $[14,(2.13)]$. But [14, Proposition 2.3.1] expresses $I_{\times}^{0, D}$ in terms of theta functions and, using this result, the Manin-Drinfeld Theorem takes the form

$$
\mathrm{AJ}_{X_{N+, N^{-}}}^{0}\left(\left[\tau_{2}\right]-\left[\tau_{1}\right]\right)=I_{\times}^{0, D}\left(\tau_{2}-\tau_{1}\right) \bmod \mathbf{q}_{\Gamma_{N^{+}, N^{-}}}^{D}=\mathrm{AJ}^{0, D}\left(\tau_{2}-\tau_{1}\right),
$$

where $\tau_{2}, \tau_{1} \in \mathcal{H}_{p},\left[\tau_{i}\right]$ is the corresponding point in $X_{N^{+}, N^{-} p}(E)$, and $\left[\tau_{2}\right]-\left[\tau_{1}\right] \in\left(\operatorname{Div}^{0} X_{N^{+}, N^{-} p}\right)(E)$ (see [14, Theorem 2.3.2]). The uniqueness follows by taking $E=\mathbb{C}_{p}$.

\section{Definite AdDitive integrals}

Let $\mathcal{D}^{0, b}\left(\mathbb{P}^{1}\left(\mathbb{Q}_{p}\right)\right)\left(\right.$ resp. $\left.\mathcal{C}_{\text {har }}^{b}(\mathcal{E}, E)\right)$ be the space of bounded distributions in $\mathcal{D}^{0}\left(\mathbb{P}^{1}\left(\mathbb{Q}_{p}\right)\right)\left(\right.$ resp. $\left.\mathcal{C}_{\text {har }}(\mathcal{E}, E)\right)$. (They have been considered in [44], [17, §2.3], [28, §3] and [35, Remark 5].) They are defined so that the morphism the induced map

$$
R: \mathcal{D}^{0, b}\left(\mathbb{P}^{1}\left(\mathbb{Q}_{p}\right)\right) \stackrel{\sim}{\longrightarrow} \mathcal{C}_{\text {har }}^{b}(\mathcal{E}, E)
$$


is an isomorphism. Furthermore, it is known that

$$
\mathcal{D}^{0, b}\left(\mathbb{P}^{1}\left(\mathbb{Q}_{p}\right)\right)^{\Gamma_{N^{+}, N^{-}}}=\mathcal{D}^{0}\left(\mathbb{P}^{1}\left(\mathbb{Q}_{p}\right)\right)^{\Gamma_{N^{+}, N^{-}}} \quad \text { and } \quad \mathcal{C}_{\text {har }}^{b}(\mathcal{E}, E)^{\Gamma_{N^{+}, N^{-}}}=\mathcal{C}_{\text {har }}(\mathcal{E}, E)^{\Gamma_{N^{+}, N^{-}}}
$$

(see $[44$, Proposition 9] and $[17, \S 2.3]$ ), so that

$$
\mathcal{D}^{0}\left(\mathbb{P}^{1}\left(\mathbb{Q}_{p}\right)\right)^{\Gamma_{N^{+}, N^{-}}} \stackrel{\sim}{\rightarrow} \mathcal{C}_{\text {har }}(\mathcal{E}, E)^{\Gamma_{N^{+}, N^{-}}}
$$

Let

$$
\ell: E^{\times} \longrightarrow E
$$

be a continuous group homomorphism, which is therefore locally analytic.

Lemma 4.1. Suppose that $\mu \in \mathcal{D}^{0, b}\left(\mathbb{P}^{1}\left(\mathbb{Q}_{p}\right)\right)$ is such that $R(\mu) \in \mathcal{C}_{\text {har }}(\mathcal{E}, \mathbb{Z})$ and that $f \in C\left(\mathbb{P}^{1}\left(\mathbb{Q}_{p}\right), E^{\times}\right)$ is such that $\ell \circ f \in \mathcal{A}\left(\mathbb{P}^{1}\left(\mathbb{Q}_{p}\right)\right)$. Then

$$
\mu(\ell \circ f)=\ell \circ(R(\mu), f) .
$$

Proof. The boundedness of $\mu \in \mathcal{D}^{0, b}\left(\mathbb{P}^{1}\left(\mathbb{Q}_{p}\right)\right)$ implies that the tautological pairing of a distribution $\mu$ against any $f^{\prime} \in \mathcal{A}_{0}\left(\mathbb{Q}_{p}\right)$ given by

$$
\mu\left(f^{\prime}\right):=\lim _{\mathcal{T}_{0} \subset \mathcal{T}} \sum_{e \in \partial \mathcal{T}_{0}} \mu\left(f^{\prime}\left(t_{e, \mathcal{T}_{0}}\right) \chi_{U_{e}}\right)=\lim _{\mathcal{T}_{0} \subset \mathcal{T}} \sum_{e \in \partial \mathcal{T}_{0}} R(\mu)(e) f^{\prime}\left(t_{e, \mathcal{T}_{0}}\right)
$$

Here, we may choose $t_{e, \mathcal{T}_{0}} \neq \infty$ for every $e$ and $\mathcal{T}_{0}$. We remark that we have

$$
R(\mu) \in \mathcal{C}_{\text {har }}(\mathcal{E}, \mathbb{Z}) \subset \mathcal{C}_{\text {har }}^{b}(\mathcal{E}, E) \stackrel{\sim}{\longleftarrow} \mathcal{D}^{0, b}\left(\mathbb{P}^{1}\left(\mathbb{Q}_{p}\right)\right)
$$

Applying (30) with $f^{\prime}=\ell \circ f$, the claim follows from the fact that $\ell$ is a continuous group homomorphism and from definition (16).

For every $\tau_{1}, \tau_{2} \in \mathcal{H}_{p}$, let us consider the function

$$
\theta_{\ell}^{\tau_{2}-\tau_{1}}(z):=\ell\left(\theta_{\times}^{\tau_{2}-\tau_{1}}(z)\right)=\ell\left(\frac{z-\tau_{2}}{z-\tau_{1}}\right), \quad z \in \mathbb{P}^{1}(\mathbb{Q}),
$$

whose equivalence class modulo $E$ (constant functions) is denoted $\left[\theta_{\ell}^{\tau_{2}-\tau_{1}}\right]$. We may then define a $\mathbf{G L}_{2}\left(\mathbb{Q}_{p}\right)$ invariant pairing

$$
I_{\ell}^{0}: \mathcal{D}^{0}\left(\mathbb{P}^{1}\left(\mathbb{Q}_{p}\right)\right) \otimes \Delta^{0}(E) \longrightarrow E
$$

by extending of the rule $I_{\ell}^{0}\left(\mu, \tau_{2}-\tau_{1}\right):=\left(\mu,\left[\theta_{\ell}^{\tau_{2}-\tau_{1}}\right]\right)$, well defined as $\mu(1)=0$. As an immediate consequence of Lemma 4.1 and (28), we deduce the commutativity of the following diagram:

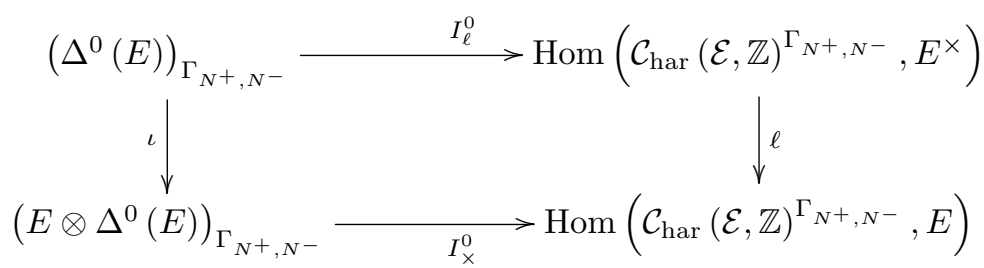

Here, $\ell$ is induced by (29). We can now complete the proof of Proposition 3.1 as follows. First, we remark that, according to $[18, \S 6.4]$, must prove that

$$
\mathbf{q}_{\Gamma_{N^{+}, N^{-}}} \subset \operatorname{Hom}\left(\mathcal{C}_{\text {har }}(\mathcal{E}, \mathbb{Z})^{\Gamma_{N^{+}, N^{-}}}, \mathbb{Q}_{p}^{\times}\right) \underset{9}{\stackrel{\operatorname{ord}}{\longrightarrow} \operatorname{Hom}\left(\mathcal{C}_{\text {har }}(\mathcal{E}, \mathbb{Z})^{\Gamma_{N^{+}, N^{-}}}, \mathbb{Z}\right) \subset \operatorname{Hom}_{\mathbb{R}}\left(\mathcal{C}_{\text {har }}(\mathcal{E}, \mathbb{Z})^{\Gamma_{N+, N^{-}}}, \mathbb{R}\right)}
$$


has finite kernel $K$ and that its image is a lattice in the classical sense ${ }^{1}$. Consider the following diagram, commutativeby (32).

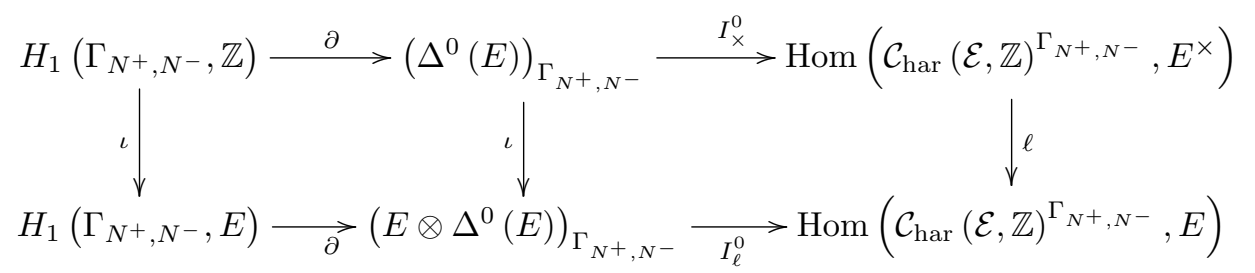

We are interested in the case $\ell=$ ord and $E=\mathbb{Q}_{p}$. The same formalism as in [28, proof of Theorem 3.11] applies in this setting with a shift by -1 in the degrees. This reduces the proof of the fact that $I_{\text {ord }}^{0} \circ \partial$ is an isomorphism to the proof that the natural boundary map

$$
\delta: \mathcal{C}_{\text {har }}\left(\mathcal{E}, \mathbb{Q}_{p}\right)^{\Gamma_{N^{+}, N^{-}}} \longrightarrow H^{1}\left(\Gamma_{N^{+}, N^{-}}, \mathbb{Q}_{p}\right)
$$

is an isomorphism. This is true thanks to [37, discussion before Theorem (3.9)]. By definition we have $\mathbf{q}_{\Gamma_{N^{+}, N^{-}}}:=\operatorname{Im}\left(I_{\times}^{0} \circ \partial\right)$ and it follows from the commutative diagram (34) and the fact that $I_{\text {ord }}^{0} \circ \partial$ is injective that $K \subset \operatorname{ker}(\iota)$. But according to the Universal Coefficients Theorem, we have

$$
\iota: H_{1}\left(\Gamma_{N^{+}, N^{-}}, \mathbb{Z}\right) \longrightarrow H_{1}\left(\Gamma_{N^{+}, N^{-}}, \mathbb{Z}\right) \otimes_{\mathbb{Z}} \mathbb{Q}_{p}=H_{1}\left(\Gamma_{N^{+}, N^{-}}, \mathbb{Q}_{p}\right)
$$

in (34). In particular we see that \# $\operatorname{ker}(\iota)<\infty$, being the torsion subgroup of the finitely generated abelian group $H_{1}\left(\Gamma_{N^{+}, N^{-}}, \mathbb{Z}\right)$. The finiteness of $K$ follows from the inclusion $K \subset \operatorname{ker}(\iota)$. It remains to show that ord $\left(\mathbf{q}_{\Gamma_{N^{+}, N^{-}}}\right)$is a lattice. Since

$$
\operatorname{ord}\left(\mathbf{q}_{\Gamma_{N^{+}, N^{-}}}\right)=\operatorname{Im}\left(\operatorname{ord} \circ I_{\times}^{0} \circ \partial\right) \subset \operatorname{Hom}\left(\mathcal{C}_{\text {har }}(\mathcal{E}, \mathbb{Z})^{\left.\Gamma_{N^{+}, N^{-}}, \mathbb{Z}\right)}\right.
$$

is finitely generated, we need only show that its rank is the $\mathbb{R}$-dimension of the codomain

$$
\operatorname{Hom}_{\mathbb{R}}\left(\mathcal{C}_{\text {har }}(\mathcal{E}, \mathbb{Z})^{\Gamma_{N^{+}, N^{-}}}, \mathbb{R}\right)=\operatorname{Hom}\left(\mathcal{C}_{\text {har }}(\mathcal{E}, \mathbb{Z})^{\Gamma_{N^{+}, N^{-}}}, \mathbb{Z}\right) \otimes_{\mathbb{Z}} \mathbb{R}
$$

of (33) or, equivalently, that it equals the $\mathbb{Q}_{p}$-dimension of $\operatorname{Hom}_{\mathbb{Q}_{p}}\left(\mathcal{C}_{\text {har }}(\mathcal{E}, \mathbb{Z})^{\Gamma_{N^{+}, N^{-}}}, \mathbb{Q}_{p}\right)$. This is true if and only if the $\mathbb{Q}_{p^{-}}$span of ord $\left(\mathbf{q}_{\Gamma_{N^{+}, N^{-}}}\right)$is

$$
\operatorname{Hom}_{\mathbb{Q}_{p}}\left(\mathcal{C}_{\text {har }}(\mathcal{E}, \mathbb{Z})^{\Gamma_{N^{+}, N^{-}}}, \mathbb{Q}_{p}\right)=\operatorname{Hom}\left(\mathcal{C}_{\text {har }}(\mathcal{E}, \mathbb{Z})^{\Gamma_{N^{+}, N^{-}}}, \mathbb{Z}\right) \otimes_{\mathbb{Z}} \mathbb{Q}_{p}
$$

But this is a consequence of the commutativity of (34), the surjectivity of $I_{\text {ord }}^{0} \circ \partial$ and (35).

We remark that

$$
I_{\text {ord }}^{0} \circ \partial, I_{\log _{0}}^{0} \circ \partial: H_{1}\left(\Gamma_{N^{+}, N^{-}}, E\right) \longrightarrow T_{0}\left(J_{\Gamma_{N^{+}, N^{-}}}\right)(E)
$$

are Hecke equivariant maps. Because we have proved that $I_{\log _{0}}^{0} \circ \partial$ is an isomorphism (and we may take $E=\mathbb{Q}_{p}$ ), the following definition makes sense (cf. [28, Corollary 3.13]). Recall that we have

$$
T_{0}\left(J_{\Gamma_{N^{+}, N^{-}}}\right)(E)=\operatorname{Hom}\left(\mathcal{C}_{\text {har }}(\mathcal{E}, \mathbb{Z})^{\left.\Gamma_{N^{+}, N^{-}}, E\right)}\right.
$$

and we write $\mathbb{T}_{\mathbb{Q}_{p}}$ for the Hecke algebra (with $\mathbb{Q}_{p}$ coefficients) acting on $T_{0}\left(J_{\Gamma_{N^{+}, N^{-}}}\right)\left(\mathbb{Q}_{p}\right)$.

Definition 4.2. The Teitelbaum $\mathcal{L}$-invariant is the unique endomorphism $\mathcal{L} \in \operatorname{End}_{\mathbb{T}_{\mathbb{Q}_{p}}}\left(T_{0}\left(J_{\Gamma_{N^{+}, N^{-}}}\right)\left(\mathbb{Q}_{p}\right)\right)$ such that

$$
I_{\log _{0}}^{0} \circ \partial=\mathcal{L} \circ I_{\text {ord }}^{0} \circ \partial .
$$

\footnotetext{
${ }^{1}$ Indeed let $K$ be the finite kernel, so that we may write $\mathbf{q}_{\Gamma_{N^{+}, N^{-}}}=K \times \mathbf{q}_{\Gamma_{N^{+}, N^{-}}}^{\prime}$ with $\mathbf{q}_{\Gamma_{N^{+}, N^{-}}}^{\prime} \subset \mathbf{q}_{\Gamma_{N^{+}, N^{-}}}$such that ord is injective on $\mathbf{q}_{\Gamma_{N}+, N^{-}}^{\prime}$ and such that the image is a lattice. Then, according to $[18, \S 6.4], \frac{\mathbf{T}_{\Gamma_{N}+, N^{-}}}{\mathbf{q}_{\Gamma_{N^{+}, N^{-}}^{\prime}}^{\prime}}$ is an analytic torus. But since $K$ is finite, $\frac{\mathbf{T}_{\Gamma_{N}+, N^{-}}}{\mathbf{q}_{\Gamma_{N}+, N^{-}}}=\left(\frac{\mathbf{T}_{\Gamma_{N^{+}, N^{-}}}}{\mathbf{q}_{\Gamma_{N^{+}, N^{-}}}^{\prime}}\right)^{K}$ exists (again by [18, §6.4]) and it is also an analytic torus.
} 
By construction of $J_{\Gamma_{N^{+}, N^{-}}}$, we have $\mathcal{L}=\mathcal{L}\left(J_{\Gamma_{N^{+}, N^{-}}}\right)$: apply (13) with

$$
j: \mathbf{q}_{\Gamma_{N^{+}, N^{-}}}:=\operatorname{Im}\left(I_{\times}^{0} \circ \partial\right) \subset \operatorname{Hom}\left(\mathcal{C}_{\text {har }}(\mathcal{E}, \mathbb{Z})^{\Gamma_{N^{+}, N^{-}}}, E^{\times}\right)
$$

and use (32) to deduce that

$$
\log _{0} \circ I_{\times}^{0} \circ \partial=I_{\log _{0}}^{0} \circ \partial=\mathcal{L} \circ I_{\text {ord }}^{0} \circ \partial=\mathcal{L} \circ \text { ord } \circ I_{\times}^{0} \circ \partial,
$$

from which we get $j_{\log _{0}}=\mathcal{L}(J) \circ j_{\text {ord }}$. We deduce from Lemma 2.1 that the formal logarithm

$$
\log _{J_{\Gamma_{N}+, N^{-}}}: J_{\Gamma_{N^{+}, N^{-}}}(E) \longrightarrow T_{0}\left(J_{\Gamma_{N^{+}, N^{-}}}\right)(E)
$$

is given by the rule

$$
\log _{J_{\Gamma^{+}}, N^{-}}([z])=\log _{0} \circ z-\mathcal{L}(\text { ord } \circ z),
$$

where $z \in \operatorname{Hom}\left(\mathcal{C}_{\text {har }}(\mathcal{E}, \mathbb{Z})^{\Gamma_{N^{+}, N^{-}}}, E^{\times}\right)$represents $[z] \in J_{\Gamma_{N^{+}, N^{-}}}(E)$.

The following result is now a consequence of (34) with $\ell=$ ord and $\ell=\log _{0}$.

Proposition 4.3. We have:

$$
\log _{J_{\Gamma_{N}+, N^{-}}} \circ \mathrm{AJ}^{0}=I_{\log _{0}}^{0} \circ \iota-\mathcal{L} \circ I_{\text {ord }}^{0} \circ \iota=: \log \mathrm{AJ}^{0} .
$$

\section{Definite ADDitive INTEGRALS: PRoOF OF THE MAIN RESUlt}

We remark that the Hecke action on the $\mathbb{Z}$ appearing in the long exact sequence (20) is Eisenstein: for a prime $q \nmid p N$, we define $t_{q}:=(q+1)-T_{q}$; then $t_{q}=0$ on it. It follows that we may regard $t_{q}$ as a morphism

$$
t_{q}:(\Delta(E))_{\Gamma_{N^{+}, N^{-}}} \rightarrow\left(\Delta^{0}(E)\right)_{\Gamma_{N^{+}, N^{-}}} .
$$

Noticing that $t_{q} \in \operatorname{End}_{\mathbb{Q}_{p}}\left(T_{0}\left(J_{\Gamma_{N^{+}, N^{-}}}\right)\right)$is invertible (because $T_{0}\left(J_{\Gamma_{N^{+}, N^{-}}}\right)$is cuspidal), we may define

$$
\log \mathrm{AJ}:=t_{q}^{-1} \circ \log \mathrm{AJ}^{0} \circ t_{q}:(\Delta(E))_{\Gamma_{N^{+}, N^{-}}} \longrightarrow T_{0}\left(J_{\Gamma_{N^{+}, N^{-}}}\right) .
$$

Proposition 5.1. The map $\log$ AJ does not depend on the choice of $q \nmid p N$ and is the unique Hecke equivariant morphism making the following diagram commutative:

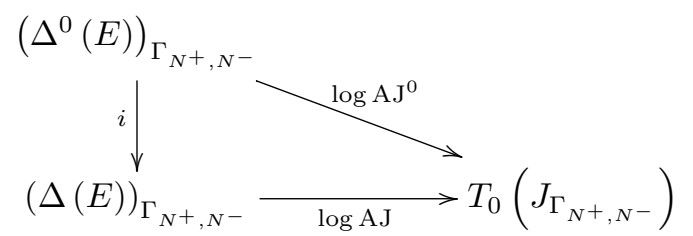

With the notation introduced before the statement of Theorem 1.1, it is given the formula

$$
(\log \mathrm{AJ})(\tau)\left(c_{\omega}\right)=\frac{d}{d \kappa}\left[\mu_{\Omega, r(\tau)}^{\omega}\langle x-\tau y\rangle^{\kappa}\right]_{\kappa=0} .
$$

Proof. The fact that $\log$ AJ is Hecke equivariant follows from the Hecke equivariance of the maps in (36). The Hecke equivariance of

$$
\log \mathbf{A J}: \tau \mapsto \frac{d}{d \kappa}\left[\mu_{\Omega, r(\tau)}\langle x-\tau y\rangle^{\kappa}\right]_{\kappa=0} \in T_{0}\left(J_{\Gamma_{N^{+}, N^{-}}}\right)
$$

can be proved as in [4, Proposition 2.18].

The fact that $\log$ AJ makes (37) commute is an easy consequence of the Hecke equivariance of $\log \mathrm{AJ}^{0}$. The fact that $\log \mathbf{A J}$ makes (37) commutative can be proved as in [4, Proposition 2.19] or, more explicitly, using [34, displayed equation after (24)].

It remains to check that there is a unique Hecke equivariant morphism making (37) commutative, from which independence of $q$ and the equality $(\log \mathrm{AJ})(\tau)=(\log \mathbf{A J})(\tau)$ will follow. Set $T:=T_{0}\left(J_{\Gamma_{N^{+}, N^{-}}}\right)$and suppose that $\Delta \in \operatorname{Hom}_{\mathbb{T}}\left((\Delta(E))_{\Gamma_{N^{+}, N^{-}}}, T\right)$ is the difference of two morphisms making (37) commutative, 
where we write $\mathbb{T}$ for the Hecke algebra. (It suffices to consider the Hecke algebra generated by the $T_{q}$ for $q \nmid p N$ for our purposes.) Then the restriction $\left.\Delta\right|_{\operatorname{Im}(i)}$ of $\Delta$ to $\operatorname{Im}(i)$ is zero. It follows from (20) that there is an exact sequence

$$
0 \longrightarrow \operatorname{Im}(i) \longrightarrow(\Delta(E))_{\Gamma_{N^{+}, N^{-}}} \stackrel{\operatorname{deg}}{\longrightarrow} \mathbb{Z} \longrightarrow 0
$$

Taking the $T$-dual yields

$$
0 \longrightarrow \operatorname{Hom}_{\mathbb{T}}(\mathbb{Z}, T) \longrightarrow \operatorname{Hom}_{\mathbb{T}}\left((\Delta(E))_{\Gamma_{N^{+}, N^{-}}}, T\right) \longrightarrow \operatorname{Hom}_{\mathbb{T}}(\operatorname{Im}(i), T)
$$

But we have $\operatorname{Hom}_{\mathbb{T}}(\mathbb{Z}, T)=0$ because $t_{q}=0$ on $\mathbb{Z}$ while it is invertible on $T$. Therefore, $\left.\Delta \mapsto \Delta\right|_{\operatorname{Im}(i)}$ is injective and $\Delta=0$ as claimed.

Our Theorem 1.1 is now a consequence of Propositions 5.1 and 4.3 and Theorem 3.2 as follows. First, Theorem 3.2 implies that, up to the identification $J_{\Gamma_{N^{+}, N^{-}}} \stackrel{\sim}{\rightarrow} J_{N^{+}, N^{-}}$, we have

$$
\mathrm{AJ}_{X_{N^{+}, N^{-}}}^{0}\left(\left[\tau_{2}\right]-\left[\tau_{1}\right]\right)=A J^{0}\left(\tau_{2}-\tau_{1}\right)
$$

for every $\tau_{2}, \tau_{1} \in \mathcal{H}_{p}$, where $\left[\tau_{i}\right]$ is the corresponding point in $X_{N^{+}, N^{-} p}(E)$. As explained in $\S 2$, the isomorphism $J_{\Gamma_{N^{+}, N^{-}}} \stackrel{\sim}{\longrightarrow} J_{N^{+}, N^{-}}$induces an identification $T_{0}\left(J_{\Gamma_{N^{+}, N^{-}}}\right)(E) \stackrel{\sim}{\longrightarrow} T_{0}\left(J_{N^{+}, N^{-}}\right)$and the formal logarithms are identified by the characterization appearijg in Lemma 2.1. We deduce from (38) that we have

$$
\log _{J_{N^{+}, N^{-}}}\left(\mathrm{AJ}_{X_{N^{+}, N^{-} p}}^{0}\left(\left[\tau_{2}\right]-\left[\tau_{1}\right]\right)\right)=\log _{J_{\Gamma_{N^{+}, N^{-}}}}\left(\mathrm{AJ}^{0}\left(\tau_{2}-\tau_{1}\right)\right) .
$$

It now follows from Proposition 4.3 and (39) that we have

$$
\log _{J_{N^{+}, N^{-p}}}\left(\mathrm{AJ}_{X_{N^{+}, N^{-p}}}^{0}\left(\left[\tau_{2}\right]-\left[\tau_{1}\right]\right)\right)=\log \mathrm{AJ}^{0}\left(\tau_{2}-\tau_{1}\right) .
$$

Recalling the definitions (8) of $\log _{J_{N^{+}, N^{-}}} A J$ and (36) of $\log$ AJ, we deduce from (40) that we have

$$
\left(\log _{J_{N+, N^{-}}} A J\right)([\tau])=(\log \mathrm{AJ})(\tau)
$$

for every $\tau \in \mathcal{H}_{p}$. Now Theorem 1.1 follows from the explicit expression of $\log$ AJ that appears in Proposition 5.1 .

We end this section explaining how the main result of [4], generalized to the case where the modular form may not have rational Fourier coefficients, follows from our Theorem 1.1. To this end, for a quadratic Dirichlet character $\chi$, we write $S_{2}\left(\Gamma_{0}(N p)\right)_{\chi(-N)=\omega_{N}, \chi(p)=a_{p}}^{\text {new }}$ for the space of those modular forms (on $\mathbf{G L}_{2}$ ) that are new and such that the eigenvalues of $W_{N}$ and $U_{p}$ acting on $f$ are $\chi(-N)$ and $\chi(p)$, respectively. Let $\mathbb{Q}^{\chi} / \mathbb{Q}$ be the extension cut out by the character $\chi$ and write $\mathbb{Q}_{\mathfrak{p}}^{\chi}$ for the $\mathfrak{p}$-adic completion at a prime $\mathfrak{p} \mid p$ (determined by a fixed embedding $\overline{\mathbb{Q}} \hookrightarrow \mathbb{C}_{p}$ ). The Fourier expansion at the $\infty$-cusp determines a $\mathbb{Q}$-rational structure on the space of modular forms using which we may consider $S_{2}\left(\Gamma_{0}(N p), \mathbb{Q}_{\mathfrak{p}}^{\chi}\right)_{\chi}^{\text {new }}(-N)=\omega_{N}, \chi(p)=a_{p}$. The conditions

$$
\chi(-N)=\omega_{N} \text { and } \chi(p)=a_{p}
$$

cut out a quotient $J_{\chi(-N)=\omega_{N}, \chi(p)=a_{p}}^{\text {new }}$ of the new part $J^{\text {new }}$ of the Picard variety $J=J_{p N, 1}$ of the modular curve.

Let $f \in S_{2}\left(\Gamma_{0}(N p)\right)_{\chi(-N)=\omega_{N}, \chi(p)=-\omega_{p}}^{\text {new }}$ and we $f_{\infty}$ be the normalized Hida eigenfamily through $f$ and write $\mathcal{L}_{f_{\infty}, \chi}$ for the Mazur-Kitagawa $p$-adic $L$-function, restricted to the central critical line. Due to the sign conditions $(41), \mathcal{L}_{f_{\infty}, \chi}$ vanishes at the weight $0:=\left[t \mapsto t^{0}=1\right]$ to order at least 2 and the association $f \mapsto \mathcal{L}_{f_{\infty}, \chi}^{\prime \prime}(0)$ gives rise to a well defined function to

$$
\mathcal{L}_{\chi}^{\prime \prime}(0): S_{2}\left(\Gamma_{0}(N p), \mathbb{Q}_{\mathfrak{p}}^{\chi}\right)_{\chi(-N)=\omega_{N}, \chi(p)=a_{p}}^{\text {new }} \longrightarrow \mathbb{Q}_{\mathfrak{p}}^{\chi} .
$$

Let us remark that it could also be regarded as a map

$$
\mathcal{L}_{\chi}^{\prime \prime}(0): H^{0}\left(X_{N p, 1 / \mathbb{Q}_{\mathfrak{p}}^{\chi}}, \Omega_{X_{N p, 1 / \mathbb{Q}_{\mathfrak{p}}^{\chi}}^{1}}\right)_{\chi(-N)=\omega_{N}, \chi(p)=a_{p}}^{\text {new }} \longrightarrow \mathbb{Q}_{\mathfrak{p}}^{\chi},
$$


it being just $\log _{J_{\chi(-N)=\omega_{N}, \chi(p)=a_{p}}^{\text {new }}}^{2}(P)$ for every $P \in\left(\mathbb{Q} \otimes J_{\chi(-N)=\omega_{N}, \chi(p)=a_{p}}^{\text {new }}\left(\mathbb{Q}^{\chi}\right)\right)^{\chi}$.

As a final piece of notation, for a $\mathbb{Q}\left[G_{\mathbb{Q} \times / \mathbb{Q}}\right]$-module $V$, we write $V^{\chi}$ for the $\chi$-component of $V$, we write $\mathbb{T}_{\chi(-N)=\omega_{N}, \chi(p)=-\omega_{p}}^{\text {new }}$ for the (rational) Hecke algebra acting on $S_{2}\left(\Gamma_{0}(N p)\right)_{\chi(-N)=\omega_{N}, \chi(p)=a_{p}}^{\text {new }}$ and, for a $\mathbb{T}_{\chi(-N)=\omega_{N}, \chi(p)=-\omega_{p}}^{\text {new }}$-module $M$ and $f \in S_{2}\left(\Gamma_{0}(N p)\right)_{\chi(-N)=\omega_{N}, \chi(p)=a_{p}}^{\text {new }}$ with companion class $[f]$, we write $M_{[f]}$ for the [f]-isotypic component. We recall that the companion class of a modular form is the set of its Galois conjugates.

We now explain give our generalization of [4, Theorem 5.4].

Theorem 5.2. Suppose that there is a prime $q$ with $q \| N$.

(1) There is an element

$$
P^{\chi} \in\left(\mathbb{Q} \otimes J_{\chi(-N)=\omega_{N}, \chi(p)=a_{p}}^{\mathrm{new}}\left(\mathbb{Q}^{\chi}\right)\right)^{\chi}
$$

and a Hecke operator $t=t_{\chi} \in \mathbb{T}_{\chi(-N)=\omega_{N}, \chi(p)=-\omega_{p}}^{n e w, \times}$ such that

$$
\mathcal{L}_{\chi}^{\prime \prime}(0)=t \cdot \log _{J_{\chi(-N)=\omega_{N}, \chi(p)=a_{p}}^{\text {new }}}^{2}\left(P^{\chi}\right) .
$$

(2) If

$$
0 \neq P_{[f]}^{\chi} \in\left(\mathbb{Q} \otimes J_{[f]}\left(\mathbb{Q}^{\chi}\right)\right)^{\chi},
$$

then

$$
\left(\mathbb{Q} \otimes J_{[f]}\left(\mathbb{Q}^{\chi}\right)\right)^{\chi}=\mathbb{T}_{[f]} \otimes \mathbb{Q} \cdot P_{[f]}^{\chi} \simeq \mathbb{T}_{[f]} \otimes \mathbb{Q},
$$

where $P_{[f]}^{\chi}$ is the $[f]$-component of $P^{\chi}$.

(3) We have $0 \neq P_{[f]}^{\chi}$ if and only if $L^{\prime}(f, \chi, 1) \neq 0$.

(4) If $t_{f} \in \mathbb{Q}(f)$ is the $f$-component of $t$, then for any quadratic Dirichlet character $\epsilon$ such that

$$
\epsilon(N)=\chi(N), \quad \epsilon(p)=-\chi(p), \quad \text { and } \quad L(f, \epsilon, 1) \neq 0,
$$

the following congruence holds

$$
t_{f} \equiv L(f, \epsilon, 1) \quad \text { in } \quad \mathbb{Q}(f)^{\times} / \mathbb{Q}(f)^{\times 2} .
$$

(Here $\mathbb{Q}(f)$ is the field generated by the Fourier coefficients of $f$.)

Proof. We sketch the required adjustments to the proof of [4, Theorem 5.4]. We will apply our Theorem 1.1 with $B=B_{q}$ the unique definite quaternion algebra of discriminant $q$. By the Jacquet-Langlands correspondence $J_{\chi(-N)=\omega_{N}, \chi(p)=a_{p}}^{\text {new }}$ appears as a quotient of $J_{N / q, q p}$ over $\mathbb{Q}$; indeed $P^{\chi}$ will be constructed from a "point" on $J_{N / q, q p}$. In order to deduce the main formula (42), we extend scalars to a field $E$ containing the $p$-adic completion of $\mathbb{Q}(f)$. We write $\omega_{f}$ for the differential associated to $f$ and $\omega_{\phi}$ for its pull-back to $J_{N / q, q p}$. We need to find an element

$$
Q^{\chi} \in\left(\mathbb{Q} \otimes J_{N / q, q p}\left(\mathbb{Q}^{\chi}\right)\right)^{\chi}
$$

whose image $P^{\chi}$ satisfies

$$
\mathcal{L}_{\chi, f_{\infty}}^{\prime \prime}(0)=t \cdot \log _{J_{\chi(-N)=\omega_{N}, \chi(p)=a_{p}}^{\text {new }}}^{2}\left(P^{\chi}\right)\left(\omega_{f}\right)=t \cdot \log _{J_{N / q, q p}}^{2}\left(Q^{\chi}\right)\left(\omega_{\phi}\right) .
$$

Applying Theorem 1.1 when $\tau=\tau_{\Psi}$ corresponds to the Heegner point attached to an embedding $\Psi$ as in $[4, \S 3.1]$ and $[34, \S 6]$, the $k_{0}=0$ case of [34, Theorem 1.1] follows formally. From here, the generalization of [4, Theorem 4.9 and Corollary 4.10] (which is the $k_{0}=0$ case of [33, Corollaries 5.26 and 5.27]) follows too. Now the proof of Theorem 5.2 follows the same pattern as that of [4, Theorem 5.4]. (See also the proof of [33, Theorem 6.1] for the minor adjustments needed to handle the case where the Fourier coefficients need not to be rational.) 


\section{The analogous ReSults IN THE INDEFinite SETting}

Suppose that $p, N^{+}$and $N^{-}$are pairwise coprime integers, with $p$ prime and $N^{-}$the squarefree product of an even number of primes. We write $B$ for the indefinite quaternion algebra over $\mathbb{Q}$ of discriminant $N^{-}$, we fix a maximal order $\mathcal{O}_{B} \subset B$ and an Eichler order $R_{0}\left(p N^{+}, N^{-}\right) \subset B$ of level $p N^{+}$, with group of invertible elements $\Gamma_{0}\left(p N^{+}, N^{-}\right)$. We choose a local isomorphism $\iota_{p}: B_{p} \stackrel{\sim}{\rightarrow} \mathbf{G L}_{2}\left(\mathbb{Q}_{p}\right)$ and we set

$$
\widetilde{\Gamma}_{N^{+}, N^{-}}:=\iota_{p}\left(R_{0}\left(p N^{+}, N^{-}\right)[1 / p]^{\times}\right) \subset \mathbf{G L}_{2}\left(\mathbb{Q}_{p}\right) \text {. }
$$

Finally, as in the definite setting, we let $\Gamma_{N^{+}, N^{-}} \subset \widetilde{\Gamma}_{N^{+}, N^{-}}$be the subgroup of norm one elements.

Consider the exact sequence

$$
0 \longrightarrow \mathcal{C}_{\text {har }}(\mathcal{E}, \mathbb{Z}) \longrightarrow \mathcal{C}_{0}(\mathcal{E}, \mathbb{Z}) \stackrel{\delta_{s}}{\longrightarrow} \mathcal{C}(\mathcal{V}, \mathbb{Z}) \longrightarrow 0,
$$

where $\delta_{s}(c)(v):=\sum_{s(e)=v} c(e)$, which induces, taking $\Gamma_{N^{+}, N^{-}}$-cohomology and applying Shapiro's Lemma (see $[28, \S 2.5])$, the exact sequence

$$
0 \longrightarrow E \longrightarrow H^{1}\left(\Gamma_{N^{+}, N^{-}}, C_{\mathrm{har}}(\mathcal{E}, \mathbb{Z})\right) \longrightarrow H^{1}\left(\Gamma_{0}\left(p N^{+}, N^{-}\right), \mathbb{Z}\right)^{p-\text { new }} \longrightarrow 0,
$$

where

$$
\begin{aligned}
& E:=\operatorname{coker}\left(\delta_{s}: C_{0}(\mathcal{E}, \mathbb{Z})^{\Gamma_{N^{+}, N^{-}}} \longrightarrow C(\mathcal{V}, \mathbb{Z})^{\Gamma_{N^{+}, N^{-}}}\right) \\
& H^{1}\left(\Gamma_{0}\left(p N^{+}, N^{-}\right), \mathbb{Z}\right)^{p-\text { new }}:=\operatorname{ker}\left(\delta_{s}: H^{1}\left(\Gamma_{N^{+}, N^{-}}, C_{0}(\mathcal{E}, \mathbb{Z})\right) \longrightarrow H^{1}\left(\Gamma_{N^{+}, N^{-}}, C(\mathcal{V}, \mathbb{Z})\right)\right) .
\end{aligned}
$$

The notation is justified by the following lemma and the fact that $H^{1}\left(\Gamma_{0}\left(p N^{+}, N^{-}\right), \mathbb{Z}\right)^{p-\text { new }}$ is identified with the $p$-new part of $H^{1}\left(\Gamma_{0}\left(p N^{+}, N^{-}\right), \mathbb{Z}\right)$, because $\delta_{s}$ corresponds to the degeneracy maps under Shapiro's isomorphism. The proof of the next result is easy and left to the reader. For a Hecke module $M$, we write $M^{c}$ for its cuspidal part (see [28, §2.4]).

\section{Lemma 6.1.}

(1) We have that $t_{q}=0$ on $E \simeq \frac{\mathbb{Z}}{(p+1) \mathbb{Z}} \oplus \mathbb{Z}$ for every $q \nmid p N$ while

$$
t_{q}: H^{1}\left(\Gamma_{0}\left(p N^{+}, N^{-}\right), \mathbb{Z}\right)^{p-\text { new }, c} \rightarrow H^{1}\left(\Gamma_{0}\left(p N^{+}, N^{-}\right), \mathbb{Z}\right)^{p-\text { new }, c}
$$

is injective on $H^{1}\left(\Gamma_{0}\left(p N^{+}, N^{-}\right), \mathbb{Z}\right)^{p-\text { new }, c} \simeq \mathbb{Z}^{g}$.

(2) There exists $E \oplus H \subset H^{1}\left(\Gamma_{N^{+}, N^{-}}, C_{\text {har }}(\mathcal{E}, \mathbb{Z})\right)$ such that $t_{q}: H \rightarrow H$ is injective for every $q \nmid p N$,

$$
H \hookrightarrow H^{1}\left(\Gamma_{0}\left(p N^{+}, N^{-}\right), \mathbb{Z}\right)^{p-\text { new }, c}
$$

is $\mathbb{Z}$-free with torsion cokernel, inducing

$$
\mathbb{Q} \cdot H=H^{1}\left(\Gamma_{N^{+}, N^{-}}, C_{h a r}(\mathcal{E}, \mathbb{Q})\right)^{c}=\mathbb{Q} \otimes_{\mathbb{Z}} H \stackrel{\sim}{\rightarrow} H^{1}\left(\Gamma_{0}\left(p N^{+}, N^{-}\right), \mathbb{Q}\right)^{p-\text { new }, c} .
$$

(3) There exists a unique $H$ which is maximal having the property stated in (2).

We fix from now on any

$$
H \hookrightarrow H^{1}\left(\Gamma_{0}\left(p N^{+}, N^{-}\right), \mathbb{Z}\right)^{p-\text { new }, c}
$$

as granted by Lemma 6.1 (2), e.g., the unique maximal $H$ of Lemma 6.1 (3). The integration pairing $I_{\times}^{0}$ gives rise, for every field $E$, to a Hecke equivariant morphism

$$
I_{\times}^{0}: H_{1}\left(\Gamma_{N^{+}, N^{-}}, \Delta^{0}(E)\right) \longrightarrow \operatorname{Hom}\left(H^{1}\left(\Gamma_{N^{+}, N^{-}}, \mathcal{C}_{\text {har }}(\mathcal{E}, \mathbb{Z})\right), E^{\times}\right) \longrightarrow \mathbf{T}_{\Gamma_{N^{+}, N^{-}}}(E),
$$

where $\mathbf{T}_{\Gamma_{N^{+}, N^{-}}}(E):=\operatorname{Hom}\left(H, E^{\times}\right)$and the second map is induced by $H \subset H^{1}\left(\Gamma_{N^{+}, N^{-}}, \mathcal{C}_{\text {har }}(\mathcal{E}, \mathbb{Z})\right)$. Note that, since $H$ is $\mathbb{Z}$-free, $\mathbf{T}_{\Gamma_{N^{+}, N^{-}}}$is indeed a rigid analytic torus, endowed with an action of the $p$-new Hecke $\mathbb{Q}$-algebra $\mathbb{T}$ acting on the weight two, $p$-new, level $p N^{+}$cusp forms on $B$. On the other hand (17) induces the exact sequence

$$
H_{2}\left(\Gamma_{N^{+}, N^{-}}, \mathbb{Z}\right) \stackrel{\partial}{\longrightarrow} H_{1}\left(\Gamma_{N^{+}, N^{-}}, \Delta^{0}(E)\right) \stackrel{i}{\longrightarrow} H_{1}\left(\Gamma_{N^{+}, N^{-}}, \Delta(E)\right) \longrightarrow H_{1}\left(\Gamma_{N^{+}, N^{-}}, \mathbb{Z}\right) .
$$

Define $\mathbf{q}_{\Gamma_{N^{+}, N^{-}}}:=\operatorname{Im}\left(I_{\times}^{0} \circ \partial\right)$. The following result is a generalization of [19, Proposition 30] (specialized to $F=\mathbb{Q})$. 
Proposition 6.2. We have that $\mathbf{q}_{\Gamma_{N^{+}, N^{-}}} \subset \mathbf{T}_{\Gamma_{N^{+}, N^{-}}}\left(\mathbb{Q}_{p}\right)$ is Hecke stable and the quotient $J_{\Gamma_{N^{+}, N^{-}}}(E)$ is represented by a rigid analytic torus $J_{\Gamma_{N}+N^{-}}$over $\mathbb{Q}_{p}$ endowed with an action of the Hecke algebra $\mathbb{T}$.

Proof. The fact that $\mathbf{q}_{\Gamma_{N^{+}, N^{-}}}$is Hecke stable follows from the fact that $\partial$ and $I_{\times}^{0}$ are Hecke equivariant. The proof of the other assertions follows exactly the same pattern as the proof of Proposition 3.1. Indeed, (28) is replaced by

$$
H^{1}\left(\Gamma_{N^{+}, N^{-}}, \mathcal{D}^{0, b}\left(\mathbb{P}^{1}\left(\mathbb{Q}_{p}\right)\right)\right)^{c} \stackrel{\sim}{\longrightarrow} H^{1}\left(\Gamma_{N^{+}, N^{-}}, \mathcal{C}_{\text {har }}(\mathcal{E}, E)\right)^{c}=E \cdot H
$$

which is [28, Theorem 3.5] (the equality follows from Lemma $6.1(2)$ ). This allows us to define the analoge of the second line appearing in (32), namely

$$
I_{\ell}^{0}: H_{1}\left(\Gamma_{N^{+}, N^{-}}, E \otimes \Delta^{0}(E)\right) \longrightarrow \operatorname{Hom}_{E}\left(H^{1}\left(\Gamma_{N^{+}, N^{-}}, \mathcal{C}_{\text {har }}(\mathcal{E}, \mathbb{Z})\right), E\right) \longrightarrow \operatorname{Hom}_{E}(H, E)
$$

where the second arrow is given by $H \subset H^{1}\left(\Gamma_{N^{+}, N^{-}}, \mathcal{C}_{\text {har }}(\mathcal{E}, \mathbb{Z})\right)$. Then Lemma 4.1 and (46) give the analogue of (32): one need only add one to the co/homology degrees. With this formal adjustment, the proof proceed along the same lines, replacing [37, discussion before Theorem (3.9)] with [28, Theorem 3.11] (specialized to the weight 2 case). See also [19, Lemma 32] (specialized to $F=\mathbb{Q}$ ), which is $[28$, Theorem 3.11 ] on the component corresponding to $f$ with rational eigenvalues, in light of $I_{\mathrm{ord}}^{0} \circ \iota=\operatorname{ord} \circ I_{\times}^{0}$ (by the analogous of (32)).

Here is the analogue of the Manin-Drinfeld theorem in this setting:

Theorem 6.3. There is a Hecke equivariant isogeny $J_{\Gamma_{N^{+}, N^{-}}} \rightarrow J_{p N^{+}, N^{-}}^{p-\text { new }} \times J_{p N^{+}, N^{-}}^{p-\text { over }} \mathbb{Q}_{p^{2}}$, where $J_{p N^{+}, N^{-}}^{p-\text { is }}$ the $p$-new quotient of the Picard variety of the indefinite Shimura curve attached to an Eichler order of level $\mathrm{pN}^{+}$in the indefinite quaternion algebra of discriminant $N^{-}$.

Proof. The action of the involution at infinity $W_{\infty}$ induces an isogeny $J_{\Gamma_{N^{+}, N^{-}}} \rightarrow J_{\Gamma_{N^{+}, N^{-}}}^{+} \times J_{\Gamma_{N^{+}, N^{-}}^{-}}$(over $\mathbb{Q}_{p}$ ); it follows from [16], extended to include the required lift to our abelian varieties in [35] (specialized to the weight 2 case), that the $\mathcal{L}$-invariant of $J_{\Gamma_{N^{+}, N^{-}}}^{ \pm}$equals the $\mathcal{L}$-invariant of $J_{p N^{+}, N^{-}}^{p-\text { new }}$, which has split multiplicative reduction over $\mathbb{Q}_{p^{2}}{ }^{2}$. By a standard argument exploiting the fact that the abelian varieties under consideration are endowed with a $\mathbb{T}$-action and their dimension is twice the dimension of $\mathbb{T}$ over $\mathbb{Q}$, it follows that there is an isogeny $J_{\Gamma_{N^{+}, N^{-}}^{ \pm}}^{ \pm} \rightarrow J_{p N^{+}, N^{-}}^{p-\text { over }} \mathbb{Q}_{p^{2}}$.

Remark 6.4. In [15] and [26], a dual construction of $J_{\Gamma_{N^{+}, N^{-}}}^{\prime}$ endowed with an isogeny $J_{\Gamma_{N^{+}, N^{-}}}^{\prime} \rightarrow J_{p N^{+}, N^{-}}^{p-\text { new }} \times$ $J_{p N^{+}, N^{-}}^{p-\text { new }}$ is described. In particular, $J_{\Gamma_{N^{+}, N^{-}}}$and $J_{\Gamma_{N^{+}, N^{-}}}^{\prime}$ are isogenous.

By definition of $\mathbf{q}_{\Gamma_{N^{+}, N^{-}}}$, (44) induces

$$
\overline{\mathrm{AJ}}^{0}: \frac{H_{1}\left(\Gamma_{N^{+}, N^{-}, \Delta^{0}}(E)\right)}{\partial\left(H_{2}\left(\Gamma_{N^{+}, N^{-}}, \mathbb{Z}\right)\right)} \longrightarrow \frac{\mathbf{T}_{\Gamma_{N^{+}, N^{-}}}(E)}{\mathbf{q}_{\Gamma_{N^{+}, N^{-}}}}=: J_{\Gamma_{N^{+}, N^{-}}}(E) .
$$

Since $H_{1}\left(\Gamma_{N^{+}, N^{-}}, \mathbb{Z}\right)$ is a finite group - it is finitely generated and $H_{1}\left(\Gamma_{N^{+}, N^{-}}, E\right)=E \otimes H_{1}\left(\Gamma_{N^{+}, N^{-}}, \mathbb{Z}\right)=$ 0 (by the universal coefficient theorem, in view of [28, Lemma 3.10]) - it has finite exponent $h$. It follows from (45) that there is a unique $\widetilde{h}$ through which $h$ factors:

We define

$$
h: H_{1}\left(\Gamma_{N^{+}, N^{-}}, \Delta(E)\right) \stackrel{\widetilde{h}}{\longrightarrow} \frac{H_{1}\left(\Gamma_{N^{+}, N^{-}, \Delta^{0}}(E)\right)}{\partial\left(H_{2}\left(\Gamma_{N^{+}, N^{-}}, \mathbb{Z}\right)\right)} \stackrel{j}{\hookrightarrow} H_{1}\left(\Gamma_{N^{+}, N^{-}}, \Delta(E)\right) .
$$

$$
\mathrm{AJ}_{h}: H_{1}\left(\Gamma_{N^{+}, N^{-}}, \Delta(E)\right) \stackrel{\widetilde{h}}{\longrightarrow} \frac{H_{1}\left(\Gamma_{N^{+}, N^{-}, \Delta^{0}}(E)\right)}{\partial\left(H_{2}\left(\Gamma_{N^{+}, N^{-}}, \mathbb{Z}\right)\right)} \stackrel{\overline{\mathrm{AJ}}^{0}}{\longrightarrow} J_{\Gamma_{N^{+}, N^{-}}}(E)
$$

\footnotetext{
${ }^{2}$ Indeed, we have $I_{\ell}^{0} \circ \iota=\ell \circ I_{\times}^{0}$ for $\ell=$ ord or $\log _{0}$ by the analogue of $(32)$. It follows that the $\mathcal{L}$-invariant of [28] considered in [35] is the $\mathcal{L}$-invariant of the monodromy module attached to $J_{\Gamma_{N+, N^{-}}}$, i.e. the $\mathcal{L}$-invariant of $J_{\Gamma_{N^{+}, N^{-}}}$. Hence, the main result of [35] gives the required lift of the main result of [16].
} 
We will need to consider the formal logarithm

$$
\log _{J_{\Gamma^{+}, N^{-}}}: J_{\Gamma_{N^{+}, N^{-}}}(E) \rightarrow T_{0}\left(J_{\Gamma_{N^{+}, N^{-}}}\right)(E) .
$$

By its construction and Lemma $6.1(2)$, the target of $\log _{J_{N_{N}+, N^{-}}}$is identified with

$$
T_{0}\left(J_{\Gamma_{N^{+}, N^{-}}}\right)(E)=\operatorname{Hom}_{E}(H, E) \stackrel{\sim}{\sim} \operatorname{Hom}_{E}\left(H^{1}\left(\Gamma_{N^{+}, N^{-}}, \mathcal{C}_{\text {har }}(\mathcal{E}, E)\right)^{c}, E\right) .
$$

On the other hand, let

$$
\frac{d}{d \kappa}[-,-]_{\kappa=0}: H^{1}\left(\Gamma_{N^{+}, N^{-}}, \mathcal{C}\left(\mathcal{V}, \mathcal{D}_{\Omega, 0}(W)\right)\right) \otimes H_{1}\left(\Gamma_{N^{+}, N^{-}}, E \otimes \Delta(E)\right) \rightarrow E
$$

be the pairing induced by [35, Lemma 17] (which is the faux Abel-Jacobi map of [21, §7.1], up to Shapiro's isomorphism, by [21, Proposition 8.1]). It is essentially induced by a rule analogous to (6). There is a cohomological family $c_{\Omega}^{\omega} \in H^{1}\left(\Gamma_{N^{+}, N^{-}}, \mathcal{D}_{\Omega, 0}(W)\right)$ passing through every $\omega \in H^{1}\left(\Gamma_{N^{+}, N^{-}}, \mathcal{C}_{\text {har }}(\mathcal{E}, E)\right)^{c}$ (see [20, Corollary 11.4]). The following result is the analogue $\mathrm{f}$ Theorem 1.1 in this indefinite setting

Theorem 6.5. For every $z \in H_{1}\left(\Gamma_{N^{+}, N^{-}}, \Delta(E)\right)$ and $\omega \in H^{1}\left(\Gamma_{N^{+}, N^{-}}, \mathcal{C}_{\text {har }}(\mathcal{E}, E)\right)^{c}$, we have

$$
\left(\log _{J_{\Gamma^{+}, N^{-}}} \mathrm{AJ}_{h}\right)(z)(\omega)=h \cdot \frac{d}{d \kappa}\left[c_{\Omega}^{\omega}, \iota(z)\right]_{\kappa=0} .
$$

Proof. Writing $\mathcal{L}$ for the $\mathcal{L}$-invariant of $J_{\Gamma_{N^{+}, N^{-}}}$, we have

$$
\log _{J_{\Gamma^{+}, N^{-}}}([x]):=\log _{0} \circ x-\mathcal{L}(\operatorname{ord} \circ x) .
$$

Since $\overline{\mathrm{AJ}}^{0}$ is induced by $I_{\times}^{0}$, we see that (recall the morphisms $I_{\ell}^{0}$ with $\ell=\log _{0}$ or ord from (46))

$$
\log _{J_{\Gamma_{N}+, N^{-}}} \circ \overline{\mathrm{AJ}}^{0}=\log _{0} \circ I_{\times}^{0}-\mathcal{L}\left(\operatorname{ord} \circ I_{\times}^{0}\right)=\left(I_{\log _{0}}^{0}-\mathcal{L} I_{\text {ord }}^{0}\right) \circ \iota,
$$

where the second equality follows from (32). We may form the following commutative diagram

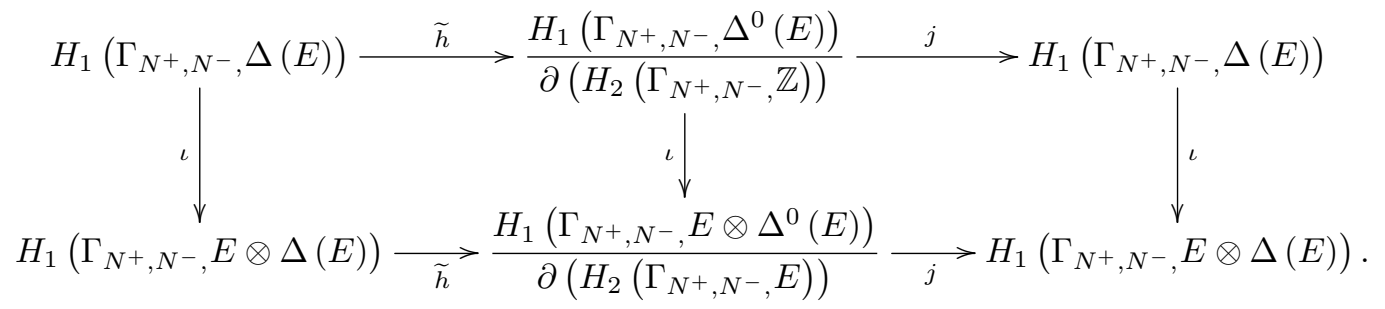

It follows that

$$
\begin{aligned}
\log _{J_{\Gamma}+, N^{-}} A J_{h} & =\log _{J_{\Gamma_{N}+, N^{-}}} \circ \overline{A J}^{0} \circ \widetilde{h}=\left(I_{\log _{0}}^{0}-\mathcal{L} I_{\text {ord }}^{0}\right) \circ \iota \circ \widetilde{h} \\
& =\left(I_{\log _{0}}^{0}-\mathcal{L} I_{\text {ord }}^{0}\right) \circ \widetilde{h} \circ \iota=\left(I_{\log _{0}}^{0}-\mathcal{L} I_{\text {ord }}^{0}\right) \circ j^{-1} \circ j \circ \widetilde{h} \circ \iota \\
& =\left(I_{\log _{0}}^{0}-\mathcal{L} I_{\text {ord }}^{0}\right) \circ j^{-1} \circ h \circ \iota=h \circ\left(I_{\log _{0}}^{0}-\mathcal{L} I_{\text {ord }}^{0}\right) \circ j^{-1} \circ \iota,
\end{aligned}
$$

the second equality following from (48).

The claim now follows from (49) and [35, Proposition 26] (or [21, Porposition 7.5] and Shapiro's lemma), giveing

$$
\left(\left(I_{\log _{0}}^{0}-\mathcal{L} I_{\text {ord }}^{0}\right) \circ j^{-1}\right)(\omega)(\iota(z))=\frac{d}{d \kappa}\left[c_{\Omega}^{\omega}, \iota(z)\right]_{\kappa=0} .
$$




\section{Derivatives of Distributions and the Coleman-Teitelbaum Cocycle}

7.1. Generalities on distributions and locally trivial bundles. Let $X$ be a locally compact, paracompact $(\Leftrightarrow$ strictly paracompact by [39, Proposition 8.7]) p-adic manifold. For a Banach $K$-algebra $\mathcal{O}$, we let $\mathcal{A}(X, \mathcal{O})$ be the space of $\mathcal{O}$-valued locally analytic functions on $X$ and let $\mathcal{D}(X, \mathcal{O}):=\mathcal{L}_{\mathcal{O}}(\mathcal{A}(X, \mathcal{O}), \mathcal{O}) \subset$ $\mathcal{L}(\mathcal{A}(X, \mathcal{O}), \mathcal{O})$, the strong $\mathcal{O}$-dual of $\mathcal{A}(X, \mathcal{O})$. Here $\mathcal{L}(V, W)$ denotes the space of continuous linear maps between the two locally convex topological spaces $V$ and $W$, with its strong topology, and $\mathcal{L}_{\mathcal{O}}(V, W)$ is its subspace of $\mathcal{O}$-linear maps, when $V$ and $W$ are given an $\mathcal{O}$-module structure, with the induced topology. If $f: X \rightarrow Y$ is a morphism of $p$-adic manifolds as above, we have maps

$$
f_{\mathcal{O}}^{*}: \mathcal{A}(Y, \mathcal{O}) \rightarrow \mathcal{A}(X, \mathcal{O}) \quad \text { and } \quad f_{*}: \mathcal{D}(X, \mathcal{O}) \rightarrow \mathcal{D}(Y, \mathcal{O}),
$$

the first being the pull-back and the second given by duality. We note that

$$
f_{*}\left(\delta_{x}^{\mathcal{O}}\right)=\delta_{f(x)}^{\mathcal{O}}, \quad \text { for every } x \in X
$$

if $\delta^{\mathcal{O}}: X \rightarrow \mathcal{D}(X, \mathcal{O})$ denotes the Dirac distribution map.

It can be shown that there are topological identifications ${ }^{3}$

$$
\mathbf{T}_{\mathcal{D}(X)}^{\mathcal{O}}: \mathcal{O} \widehat{\otimes} \mathcal{D}(X) \stackrel{\sim}{\longrightarrow} \mathcal{D}(X, \mathcal{O})
$$

and

such that

$$
\mathbf{P}_{\mathcal{D}\left(X_{1}\right), \mathcal{D}\left(X_{2}\right)}^{\mathcal{O}_{1}, \mathcal{O}_{2}}: \mathcal{D}\left(X_{1}, \mathcal{O}_{1}\right) \widehat{\otimes}_{\iota} \mathcal{D}\left(X_{2}, \mathcal{O}_{2}\right) \stackrel{\sim}{\longrightarrow} \mathcal{D}\left(X_{1} \times X_{2}, \mathcal{O}_{1} \widehat{\otimes} \mathcal{O}_{2}\right)
$$

$\mathbf{T}_{\mathcal{D}(X)}^{\mathcal{O}}\left(1_{\mathcal{O}} \widehat{\otimes} \delta_{x}\right)=\delta_{x}^{\mathcal{O}} \quad$ and $\quad \mathbf{P}_{\mathcal{D}\left(X_{1}\right), \mathcal{D}\left(X_{2}\right)}^{\mathcal{O}_{1}, \mathcal{O}_{2}}\left(\delta_{x_{1}}^{\mathcal{O}_{1}} \widehat{\otimes}_{\iota} \delta_{x_{2}}^{\mathcal{O}_{2}}\right)=\delta_{\left(x_{1}, x_{2}\right)}^{\mathcal{O}_{1} \otimes \mathcal{O}_{2}}$

Since the $K$-span of $\left\{\delta_{x}: x \in X\right\}$ is dense in $\mathcal{D}(X)$ (use strict paracompactness in order to reduce to the compact case [40, Lemma 3.1]), we also deduce that the $\mathcal{O}$-span of $\left\{\delta_{x}^{\mathcal{O}}: x \in X\right\}$ is dense in $\mathcal{D}(X, \mathcal{O})$ :

$$
\overline{\mathcal{O}\left\{\delta_{x}^{\mathcal{O}}: x \in X\right\}}=\mathcal{D}(X, \mathcal{O})
$$

It follows from (52) that the properties in (50) and (51) characterize the respective maps.

When $X$ is compact, then $\mathcal{A}(X)$ is the inductive $\operatorname{limit} \lim V_{n}$ of Banach spaces $V_{n}$ with injective and compact transition maps, while $\mathcal{D}(X)$ is the inverse $\operatorname{limit} \underset{\leftarrow n}{\lim _{\leftarrow}} V_{n}^{\prime}$ of Banach spaces of $V_{n}^{\prime}$ by compact and dense transition maps (namely $V_{n}^{\prime}=\mathcal{L}\left(V_{n}, K\right)$ ). These well known facts can be deduced from [38, Lemma 16.4, Proposition 16.5 and Proposition 16.10], noticing that $\lim _{\leftarrow} V_{n}^{\prime} \rightarrow V_{n}$ has dense image if the transition maps have this property. It follows from loc. cit. that both $\mathcal{A} \stackrel{\leftarrow}{(X)}$ and $\mathcal{D}(X)$ mutually dual, hence reflexive. We call such a locally convex space $V:=\lim _{n \rightarrow} V_{n}$ (resp. $V^{\prime}:=\lim _{\leftarrow n} V_{n}^{\prime}$ ) a space of compact type (resp. a nuclear Fréchet space). Because we may write $X=\bigsqcup_{i} X_{i}$ where $X_{i}$ is open and compact, we see that $\mathcal{A}(X)=\prod_{i} \mathcal{A}\left(X_{i}\right)$ (by [39, Proposition 12.5]) and, taking duals, yields $\mathcal{D}(X)=\bigoplus_{i} \mathcal{D}\left(X_{i}\right)$ (the reader may check that the canonical map $\bigoplus_{i} \mathcal{L}\left(V_{i}, W\right) \rightarrow \mathcal{L}\left(\prod_{i} V_{i}, W\right)$ is a topological identification, when $W$ is normed).

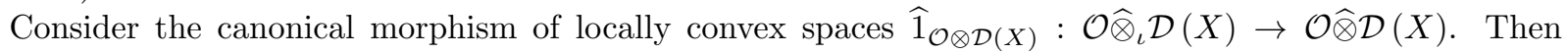
$\mathcal{D}(X)=\bigoplus_{i} \mathcal{D}\left(X_{i}\right)$ and we may form the following commutative diagram:

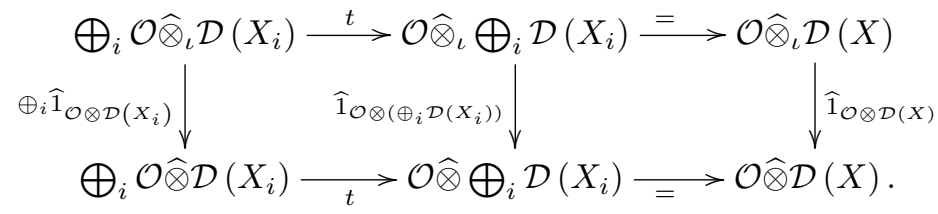

Since $\mathcal{O}$ is a Banach space, it is not difficult to see that the canonical morphisms $t$ are topological identifications. On the other hand, $\widehat{1}_{\mathcal{O} \otimes \mathcal{D}\left(X_{i}\right)}$ is a topological identification because $\mathcal{O}$ and $\mathcal{D}\left(X_{i}\right)$ are Fréchet spaces; therefore, we may apply [38, Proposition 17.6]. We have proved the following remark:

\footnotetext{
${ }^{3}$ We write $V \otimes_{\iota} W$ (resp. $V \otimes W$ ) to denote $V \otimes_{K} W$ with the inductive (resp. projective) tensor topology. Also, a morphism $V \rightarrow W$ is a continuous, $K$-linear if $V$ and $W$ are locally convex spaces. Completion means Hausdorff completion.
} 
Remark 7.1. The canonical morphism $\widehat{1}_{\mathcal{O} \otimes \mathcal{D}(X)}: \mathcal{O} \widehat{\otimes}_{\iota} \mathcal{D}(X) \rightarrow \mathcal{O} \widehat{\otimes} \mathcal{D}(X)$ is a topological $\mathcal{O}$-linear identification.

Suppose, from now on, that a locally compact, paracompact, $p$-adic Lie group $T$ acts on $X$ by means of a locally analytic map $a: T \times X \rightarrow X$. The left action of $T$ on $\mathcal{D}(X)$ can be extended, with respect to $\delta .: T \rightarrow \mathcal{D}(X)$, to a left action of $\mathcal{D}(T)$ making $\mathcal{D}(X)$ a $\mathcal{D}(T)$-module under the convolution product:

$$
(\nu \cdot \mu)(F)=\mathbf{P}_{\mathcal{D}(T), \mathcal{D}(X)}(\nu \widehat{\otimes} \mu)(F \circ a) \text {, where } \nu \in \mathcal{D}(T), \mu \in \mathcal{D}(X) \text { and } F \in \mathcal{A}(X) .
$$

More formally, the multiplication is given by the separately continuous morphism

$$
\mathcal{D}(T) \otimes_{\iota} \mathcal{D}(X) \stackrel{\mathbf{P}_{\mathcal{D}(T), \mathcal{D}(X)}^{\longrightarrow}}{\longrightarrow} \mathcal{D}(T \times X) \stackrel{a_{*}}{\rightarrow} \mathcal{D}(X) .
$$

We note the formula

$$
\delta_{t} \cdot \delta_{x}=\delta_{t x} \quad \text { for } \quad t \in T \text { and } x \in X,
$$

characterizes the multiplication law, thanks to (51). Since (54) characterizes the respective maps, upon setting $X=T$ we obtain a $K$-algebra structure on $\mathcal{D}(T)$ (with identity element $\delta_{1}$ ) and that, for a general $X$, we get a $\mathcal{D}(T)$-module structure on $\mathcal{D}(X)$. Note that, when $T$ and $X$ are compact, the multiplication law is continuous and, hence, $\mathcal{D}(T)$ is a locally convex $K$-algebra and $\mathcal{D}(X)$ is a locally convex $\mathcal{D}(T)$-module.

We write $\operatorname{Hom}_{\mathcal{A}}\left(T, \mathcal{O}^{\times}\right)$for the subgroup of $\operatorname{Hom}\left(T, \mathcal{O}^{\times}\right)$consisting ofthose group homomorphisms whose composition with the inclusion $\mathcal{O}^{\times} \subset \mathcal{O}$ belongs to $\mathcal{A}(T, \mathcal{O})$; give $\operatorname{Hom}_{\mathcal{A}}\left(T, \mathcal{O}^{\times}\right) \subset \mathcal{A}(T, \mathcal{O})$ the subspace topology. We let $\operatorname{Hom}_{\mathcal{L}}(\mathcal{D}(T), \mathcal{O}) \subset \mathcal{L}(\mathcal{D}(T), \mathcal{O})$ be the subspace consisting of those morphisms of locally convex spaces that are also morphisms of $K$-algebras ${ }^{4}$.

Lemma 7.2. The map $C^{\mathcal{O}}: \operatorname{Hom}_{\mathcal{L}}(\mathcal{D}(T), \mathcal{O}) \rightarrow \operatorname{Hom}_{\mathcal{A}}\left(T, \mathcal{O}^{\times}\right)$defined by the rule $C^{\mathcal{O}}(\mathbf{k})(t):=\mathbf{k}\left(\delta_{t}\right)$ is a well defined topological identification.

Proof. By [40, Theorem 2.2] we have to prove that $C^{\mathcal{O}}(\mathbf{k}) \in \operatorname{Hom}_{\mathcal{A}}\left(T, \mathcal{O}^{\times}\right)$if and only if $\mathbf{k} \in \operatorname{Hom}_{\mathcal{L}}(\mathcal{D}(T), \mathcal{O})^{5}$. We claim that, for a continuous $K$-linear morphism $\mathbf{k} \in \mathcal{L}(\mathcal{D}(T), \mathcal{O})$ to belong to $\operatorname{Hom}_{\mathcal{L}}(\mathcal{D}(T), \mathcal{O})$, it is necessary and sufficient that $\mathbf{k}\left(\delta_{1}\right)=1$ and $\mathbf{k}\left(\delta_{t_{1}} \cdot \delta_{t_{2}}\right)=\mathbf{k}\left(\delta_{t_{1}}\right) \mathbf{k}\left(\delta_{t_{2}}\right)$. Indeed we note that both the left and the right hand sides of the equality $\mathbf{k}\left(\nu_{1} \cdot \nu_{2}\right)=\mathbf{k}\left(\nu_{1}\right) \mathbf{k}\left(\nu_{2}\right)$ are linear in the variables $\nu_{1}$ and $\nu_{2}$. Furthermore, if we fix one of these variables, the two resulting functions are $K$-linear and continuous in the remaining variable thanks to (53), showing that the multiplication law is separately continuous. Hence, the claimed equality $\mathbf{k}\left(\nu_{1} \cdot \nu_{2}\right)=\mathbf{k}\left(\nu_{1}\right) \mathbf{k}\left(\nu_{2}\right)$ follows from the identity $\mathbf{k}\left(\delta_{t_{1}} \cdot \delta_{t_{2}}\right)=\mathbf{k}\left(\delta_{t_{1}}\right) \mathbf{k}\left(\delta_{t_{2}}\right)$ and $(52)$. Since $\mathbf{k}\left(\delta_{t_{1} t_{2}}\right)=\mathbf{k}\left(\delta_{t_{1}} \cdot \delta_{t_{2}}\right)$ by $(54)$, the claim follows.

Suppose that we are given a map $\mathbf{k} \in \operatorname{Hom}_{\mathcal{L}}(\mathcal{D}(T), \mathcal{O})$. By Lemma 7.2 its restriction to $\delta .: T \rightarrow \mathcal{D}(T)$ gives rise to a locally analytic group homomorphism $(\cdot)^{\mathbf{k}}: T \rightarrow \mathcal{O}^{\times}$sending $t$ to $t^{\mathbf{k}}:=\mathbf{k}\left(\delta_{t}\right)$. We define the following closed sub $\mathcal{O}$-module of $\mathcal{A}(X, \mathcal{O})^{6}$ :

$$
\mathcal{A}_{\mathbf{k}}(X)=\mathcal{A}(X, \mathbf{k})=\left\{F \in \mathcal{A}(X, \mathcal{O}): F(t x)=t^{\mathbf{k}} F(x)\right\} .
$$

Viewing both $\mathcal{O}$ and $\mathcal{D}(X)$ as $\mathcal{D}(T)$-modules by means of $\mathbf{k}$ and, respectively, the convolution product, we may define

$$
\mathcal{D}_{\mathbf{k}}(X):=\mathcal{O} \widehat{\otimes}_{\mathbf{k}} \mathcal{D}(X) \text { and } \mathcal{D}(X, \mathbf{k}):=\mathcal{L}_{\mathcal{O}}\left(\mathcal{A}_{\mathbf{k}}(X), \mathcal{O}\right) .
$$

We assume, further, that $X$ is endowed with a right action by a semigroup $\Sigma$ with the property that the mapping $\sigma: X \rightarrow X$ is locally analytic for every $\sigma \in \Sigma$. There are induced left (resp. right) actions of $\Sigma$ on the spaces of locally analytic functions (resp. distributions) defined by the rule $(\sigma F)(x):=\sigma^{*}(F)(x)=F(x \sigma)$

\footnotetext{
${ }^{4}$ Since the multiplication law $\mathcal{D}(T) \times \mathcal{D}(T) \rightarrow \mathcal{D}(T)$ is only separately continuous, $\mathcal{D}(T)$ is not a locally convex algebra. When $T$ is compact the multiplication law is continuous and $\operatorname{Hom}_{\mathcal{L}}(\mathcal{D}(T), \mathcal{O})$ is the space of morphisms of locally convex algebras.

${ }^{5}$ We remark that [40, Theorem 2.2] can be refined to a bicontinuous isomorphism and extended to the case where the manifold is strictly paracompact.

${ }^{6}$ Suppose that $\left(F_{i}\right) \rightarrow F$ is a net in $\mathcal{A}_{\mathbf{k}}(X)$ converging to $F \in \mathcal{A}(X, \mathcal{O})$. If $t \in T$ let $t: X \rightarrow X$ be the locally analytic map given by left multiplication by $t$, so that $t^{*}\left(F_{i}\right) \rightarrow t^{*}(F)$. Since the multiplication in $\mathcal{O}$ is separately continuous, $G \mapsto t^{\mathbf{k}_{2}} G$ is continuous and $t^{\mathbf{k}_{2}} F_{i} \rightarrow t^{\mathbf{k}_{2}} F$. Since $t^{*}\left(F_{i}\right)=t^{\mathbf{k}_{2}} F_{i}$ we deduce that $t^{*}(F)=t^{\mathbf{k}_{2}} F$ for every $t \in T$, meaning that $F \in \mathcal{A}_{\mathbf{k}}(X)$.
} 
(resp. $\left.(\Lambda \sigma)(F):=\left(\sigma_{*}(\Lambda)\right)(F)=\Lambda(\sigma F)\right)$. We will assume that the right $\Sigma$-action is compatible with the left $T$-action in the sense that $t(x \sigma)=(t x) \sigma$ for all $t \in T, x \in X$, and $\sigma \in \Sigma$. It follows that $\Sigma$ acts on $\mathcal{A}_{\mathbf{k}}(X), \mathcal{D}_{\mathbf{k}}(X)$ and $\mathcal{D}(X, \mathbf{k})$. These spaces of distributions are related by the following lemma.

Lemma 7.3. There is a unique morphism $\mathbf{T}_{\mathcal{D}(X)}^{\mathbf{k}}$ of locally convex spaces making the following diagram commutative:

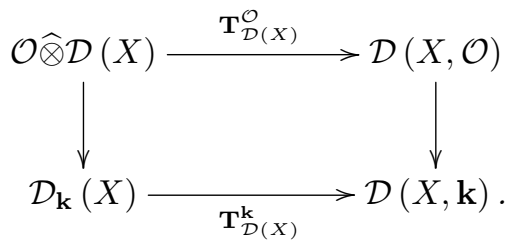

The morphism $\mathbf{T}_{\mathcal{D}(X)}^{\mathbf{k}}$ is $(\mathcal{O}, \Sigma)$-equivariant.

Proof. We first need to prove that, if $\alpha \in \mathcal{O}, \mu \in \mathcal{D}(X)$ and $\nu \in \mathcal{D}(T)$, then

$$
\mathbf{T}_{\mathcal{D}(X)}^{\mathcal{O}}(\alpha \mathbf{k}(\nu) \widehat{\otimes} \mu)=\mathbf{T}_{\mathcal{D}(X)}^{\mathcal{O}}(\alpha \widehat{\otimes}(\nu \cdot \mu)) \text { on } \mathcal{A}_{\mathbf{k}}(X) .
$$

By definition $F \in \mathcal{A}_{\mathbf{k}}(X)$ if and only if

$$
\delta_{t x}^{\mathcal{O}}(F)=F(t x)=t^{\mathbf{k}} F(x)=\left(\mathbf{k}\left(\delta_{t}\right) \delta_{x}^{\mathcal{O}}\right)(F) .
$$

By (54) and (51) we have $\mathbf{T}_{\mathcal{D}(X)}^{\mathcal{O}}\left(1 \widehat{\otimes}\left(\delta_{t} \cdot \delta_{x}\right)\right)=\mathbf{T}_{\mathcal{D}(X)}^{\mathcal{O}}\left(1 \widehat{\otimes} \delta_{t x}\right)=\delta_{t x}^{\mathcal{O}}$ and $\mathbf{T}_{\mathcal{D}(X)}^{\mathcal{O}}\left(\mathbf{k}\left(\delta_{t}\right) \widehat{\otimes} \delta_{x}\right)=\mathbf{k}\left(\delta_{t}\right) \delta_{x}^{\mathcal{O}}$. We deduce, by $\mathcal{O}$-linearity, the identity $\mathbf{T}_{\mathcal{D}(X)}^{\mathcal{O}}\left(\alpha \mathbf{k}\left(\delta_{t}\right) \widehat{\otimes} \delta_{x}^{R}\right)=\mathbf{T}_{\mathcal{D}(X)}^{\mathcal{O}}\left(\alpha \widehat{\otimes}\left(\delta_{t} \cdot \delta_{x}\right)\right)$ on $\mathcal{A}_{\mathbf{k}}(X)$. We note that both the left and right hand sides of (56) are linear in the variables $\mu$ and $\nu$. Furthermore, if we fix one of these variables, the two resulting functions are continuous in the remaining variable thanks to (53), showing that the multiplication law is separately continuous. Hence, (56) follows from (52). The existence and uniqueness of $\mathbf{T}_{\mathcal{D}(X)}^{\mathbf{k}}$ follows. Since $\mathcal{O} \widehat{\otimes} \mathcal{D}(X) \rightarrow \mathcal{D}_{\mathbf{k}}(X)$ is surjective and all the arrows other than $\mathbf{T}_{\mathcal{D}(X)}^{\mathbf{k}}$ in (55) are $(\mathcal{O}, \Sigma)$-equivariant, $\mathbf{T}_{\mathcal{D}(X)}^{\mathbf{k}}$ is equivariant as well.

We note that, writing $1 \widehat{\otimes}_{\mathbf{k}} \delta_{x} \in \mathcal{D}_{\mathbf{k}}(X)$ for the image of $1 \widehat{\otimes} \delta_{x} \in \mathcal{O} \widehat{\otimes} \mathcal{D}(X)$ and $\delta_{x}^{\mathbf{k}} \in \mathcal{D}(X, \mathbf{k})$ for the evaluation at $x$ morphism (the image of $\delta_{x}^{\mathcal{O}} \in \mathcal{D}(X, \mathcal{O})$ ), (51) implies that the morphism $\mathbf{T}_{\mathcal{D}(X)}^{\mathbf{k}}$ appearing in $(55)$ can be characterized by the formula

$$
\mathbf{T}_{\mathcal{D}(X)}^{\mathbf{k}}\left(1 \widehat{\otimes}_{\mathbf{k}} \delta_{x}\right)=\delta_{x}^{\mathbf{k}} \text { for every } x \in X .
$$

We say that $\pi: X \rightarrow T \backslash X=: \bar{X}$ is a trivial $T$-bundle if the quotient $\bar{X}:=T \backslash X$ has the structure of a locally compact, paracompact, $p$-adic manifold ${ }^{7}$ and there exists a locally analytic section $s: \bar{X} \rightarrow X$ of $\pi$ such that the locally analytic mapping $h=h_{s}: T \times \bar{X} \rightarrow X$ given by $h(t, \bar{x}):=t s(\bar{x})$ is a locally analytic isomorphism. Let

$$
s^{*}: \mathcal{A}(X, \mathcal{O}) \rightarrow \mathcal{A}(\bar{X}, \mathcal{O})
$$

be the pull-back map on functions. We let $\mathbf{k} \in \operatorname{Hom}_{\mathcal{L}}(\mathcal{D}(T), \mathcal{O})$ in the following discussion.

Lemma 7.4. The map $s^{*}$ restricts to an topological identification $s^{*}=s_{\mathbf{k}}^{*}: \mathcal{A}_{\mathbf{k}}(X) \stackrel{\sim}{\rightarrow} \mathcal{A}(\bar{X}, \mathcal{O})$.

Proof. Define $r=r_{\mathbf{k}}: \mathcal{A}(\bar{X}, \mathcal{O}) \rightarrow \mathcal{A}_{\mathbf{k}}(X)$ by $r(f)(h(t, \bar{x}))=t^{\mathbf{k}} f(\bar{x})$. We claim that $r$ is the inverse of $s^{*}$. If $f \in \mathcal{A}(\bar{X}, \mathcal{O}), F \in \mathcal{A}_{\mathbf{k}}(X), \bar{x} \in \bar{X}$ and $t \in T$, then

$$
\begin{aligned}
& s^{*}(r(f))(\bar{x})=r(f)(s(\bar{x}))=r(f)(h(1, \bar{x}))=f(\bar{x}) \text { and } \\
& r\left(s^{*}(F)\right)(h(t, \bar{x}))=t^{\mathbf{k}} s^{*}(F)(\bar{x})=t^{\mathbf{k}} F(s(\bar{x}))=F(t s(\bar{x}))=F(h(t, \bar{x})) .
\end{aligned}
$$

\footnotetext{
${ }^{7}$ According to [32, Part II, Ch. III $\S 12$ Theorem 1] there exists at most one $p$-adic manifold structure on $\bar{X}$ making $\pi: X \rightarrow \bar{X}$ a topological quotient in such a way that $\pi$ is a submersion. See [32, Part II, Ch. III $\S 12$ Theorem 2] for natural conditions granting the existence of such a quotient.
} 
We now claim that $r$ is continuous. Indeed we have

$$
\left(h^{-1}\right)^{*}\left(C^{\mathcal{O}}(\mathbf{k}) \cdot f\right)(h(t, \bar{x}))=\left(C^{\mathcal{O}}(\mathbf{k}) \cdot f\right)(t, \bar{x})=C^{\mathcal{O}}(\mathbf{k})(t) f(\bar{x})=t^{\mathbf{k}} f(\bar{x})=r(f)(h(t, \bar{x})),
$$

showing that $r(f)=\left(h^{-1}\right)^{*}\left(C^{\mathcal{O}}(\mathbf{k}) \cdot f\right)$ where $\left(h^{-1}\right)^{*}: \mathcal{A}(T \times \bar{X}, \mathcal{O}) \rightarrow \mathcal{A}(X, \mathcal{O})$ is the continuous morphism induced by $h^{-1}: X \rightarrow T \times \bar{X}$. The claim now follows from the general fact that the multiplication map

$$
\cdot: \mathcal{A}(Y, \mathcal{O}) \otimes \mathcal{A}(Y, \mathcal{O}) \longrightarrow \mathcal{A}(Y \times Y, \mathcal{O} \widehat{\otimes} \mathcal{O}) \longrightarrow \mathcal{A}(Y, \mathcal{O} \widehat{\otimes} \mathcal{O}) \longrightarrow \mathcal{A}(Y, \mathcal{O})
$$

is continuous for every strictly paracompact manifold $Y$. Here the first arrow is characterized by the fact that it maps $F_{1} \otimes F_{2}$ to $\left(y_{1}, y_{2}\right) \mapsto F_{1}\left(y_{1}\right) \widehat{\otimes} F_{2}\left(y_{2}\right)$, the second arrow is obtained by pull-back along the diagonal and the third is induced by the multiplication map $\mathcal{O} \widehat{\otimes} \mathcal{O} \rightarrow \mathcal{O}$. They are all continuous (and indeed the first extends to a topological isomorphism on Hausdorff completions), from which we deduce that · is continuous.

Recall our assumption that $X$ admits a right action by a semigroup $\Sigma$ compatible with the left $T$-action. For each $x \in X$, there is a unique $j_{s}(x) \in T$ such that $x=j_{s}(x) s(\pi(x))$. Note that $j_{s}: X \rightarrow T$ is locally analytic because $h\left(j_{s}(x), \pi(x)\right)=j_{s}(x) s(\pi(x))=x$, implying $j_{s}=\operatorname{pr}_{T} \circ h^{-1}$ where $\operatorname{pr}_{T}: T \times \bar{X} \rightarrow T$ is the canonical projection. In particular,

$$
j_{s}(\cdot, \cdot): \bar{X} \times \Sigma \longrightarrow T, \quad j_{s}(\bar{x}, \sigma):=j_{s}(s(\bar{x}) \sigma)
$$

is such that $j_{s}(\cdot, \sigma): \bar{X} \rightarrow T$ is locally analytic for every fixed $\sigma \in \Sigma$ because $j_{s}(\cdot, \sigma)=j_{s} \circ \sigma \circ s$. We may therefore define a left weight $\mathbf{k}$ action of $\Sigma$ on $\mathcal{A}(\bar{X}, \mathcal{O})$ by the rule

$$
(\sigma \cdot \mathbf{k}, s f)(\bar{x}):=j_{s}(\bar{x}, \sigma)^{\mathbf{k}} f(\bar{x} \sigma) .
$$

Lemma 7.5. The map $s^{*}: \mathcal{A}_{\mathbf{k}}(X) \rightarrow \mathcal{A}(\bar{X}, \mathcal{O})$ is $\Sigma$-equivariant.

Proof. Suppose that $F \in \mathcal{A}_{\mathbf{k}}(X), \bar{x} \in \bar{X}$ and $\sigma \in \Sigma$. By definition $s(\bar{x}) \sigma=j_{s}(s(\bar{x}) \sigma) s(\pi(s(\bar{x}) \sigma))$ and, since $\pi$ is $\Sigma$-equivariant, $\pi(s(\bar{x}) \sigma)=\pi(s(\bar{x})) \sigma=\bar{x} \sigma$. It follows that $s(\bar{x}) \sigma=j_{s}(s(\bar{x}) \sigma) s(\bar{x} \sigma)$ and then

$$
\begin{aligned}
s^{*}(\sigma F)(\bar{x}) & =(\sigma F)(s(\bar{x}))=F(s(\bar{x}) \sigma)=F\left(j_{s}(s(\bar{x}) \sigma) s(\bar{x} \sigma)\right)=j_{s}(s(\bar{x}) \sigma)^{\mathbf{k}} F(s(\bar{x} \sigma)) \\
& =j_{s}(s(\bar{x}) \sigma)^{\mathbf{k}} s^{*}(F)(\bar{x} \sigma)=\left(\sigma \cdot \mathbf{k}, s s^{*}(F)\right)(\bar{x}) .
\end{aligned}
$$

For each $\sigma \in \Sigma$, we have defined $\sigma_{\cdot \mathbf{k}, s}: \mathcal{A}(\bar{X}, \mathcal{O}) \rightarrow \mathcal{A}(\bar{X}, \mathcal{O})$. We note that this map is continuous because $C^{\mathcal{O}}(\mathbf{k})\left(j_{s}(\cdot, \sigma)(\bar{x})\right)=j_{s}(\bar{x}, \sigma)^{\mathbf{k}}$, so that

$$
\sigma \cdot \mathbf{k}, s=\left(C^{\mathcal{O}}(\mathbf{k}) \circ j_{s}(\cdot, \sigma)\right) \cdot \sigma^{*}(f)
$$

and the continuity is a consequence of the continuity of (57). It follows that we may define a weight $\mathbf{k}$ action of $\Sigma$ on $\mathcal{D}(\bar{X}, \mathcal{O})$ by the rule

$$
(\mu \cdot \mathbf{k}, s)(f):=\mu(\sigma \cdot \mathbf{k}, s f) .
$$

By Lemmas 7.4 and 7.5 there is an $(\mathcal{O}, \Sigma)$-equivariant topological identification $s^{*}: \mathcal{A}_{\mathbf{k}}(X) \stackrel{\sim}{\rightarrow} \mathcal{A}(\bar{X}, \mathcal{O})$. Taking the $\mathcal{O}$-duals it yields an $(\mathcal{O}, \Sigma)$-equivariant topological identification

$$
s_{*}=s_{*}^{\mathbf{k}}: \mathcal{D}(\bar{X}, \mathcal{O}) \stackrel{\sim}{\rightarrow} \mathcal{D}(X, \mathbf{k}) .
$$

Proposition 7.6. The morphism $\mathbf{T}_{\mathcal{D}(X)}^{\mathbf{k}}$ is a $(\mathcal{O}, \Sigma)$-equivariant topological identification $\mathbf{T}_{\mathcal{D}(X)}^{\mathbf{k}}: \mathcal{D}_{\mathbf{k}}(X) \stackrel{\sim}{\rightarrow}$ $\mathcal{D}(X, \mathbf{k})$.

Proof. We let $T$ act on $T \times \bar{X}$ by the rule $t_{1}\left(t_{2}, \bar{x}\right):=\left(t_{1} t_{2}, \bar{x}\right)$ : since $h\left(t_{1}\left(t_{2}, \bar{x}\right)\right)=t_{1} t_{2} s(\bar{x})=t_{1} h\left(t_{2}, \bar{x}\right)$ we have

$$
h_{*}^{\mathbf{k}}: \mathcal{O} \widehat{\otimes}_{\mathbf{k}} \mathcal{D}(T \times \bar{X}) \rightarrow \mathcal{O} \widehat{\otimes}_{\mathbf{k}} \mathcal{D}(X)=: \mathcal{D}_{\mathbf{k}}(X) .
$$

This map is an $\mathcal{O}$-linear isomorphism by a general functoriality principle, because $\left(1_{T}, h\right):(T, T \times \bar{X}) \rightarrow$ $(T, X)$ is a locally analytic isomorphism of $T$-bundles. Indeed, $h_{*}^{\mathbf{k}}$ is induced by $1 \widehat{\otimes} h_{*}$ and it is characterized by

$$
h_{*}^{\mathbf{k}}\left(\alpha \widehat{\otimes}_{\mathbf{k}} \mu\right)=\alpha \widehat{\otimes}_{\mathbf{k}} h_{*}(\mu) .
$$


Consider the topological identification $\mathbf{P}:=\mathbf{P}_{\mathcal{D}(T), \mathcal{D}(X)}: \mathcal{D}(T) \widehat{\otimes}_{\iota} \mathcal{D}(\bar{X}) \rightarrow \mathcal{D}(T \times \bar{X})$. Note that it is $\mathcal{D}(T)$-linear: indeed, by (54) and (51), we have

$$
\mathbf{P}\left(\left(\delta_{t_{1}} \cdot \delta_{t_{2}}\right) \widehat{\otimes} \delta_{\bar{x}}\right)=\mathbf{P}\left(\delta_{t_{1} t_{2}} \widehat{\otimes} \delta_{\bar{x}}\right)=\delta_{\left(t_{1} t_{2}, \bar{x}\right)}=\delta_{t_{1}\left(t_{2}, \bar{x}\right)}=\delta_{t_{1}} \cdot \delta_{\left(t_{2}, \bar{x}\right)} .
$$

Both sides of this equation are linear in the involved variables and, if we fix all but one, the resulting function is continuous in the remaining variable thanks to (53) showing that the multiplication laws are separately continuous. The $\mathcal{D}(T)$-linearity follows from this remark and (52) by the above equation. We deduce that

$$
1_{\mathcal{O}} \widehat{\otimes}_{\mathbf{k}} \mathbf{P}: \mathcal{O} \widehat{\otimes}_{\mathcal{L}} \mathcal{D}(\bar{X})=\mathcal{O} \widehat{\otimes}_{\mathbf{k}} \mathcal{D}(T) \widehat{\otimes}_{\iota} \mathcal{D}(\bar{X}) \longrightarrow \mathcal{O} \widehat{\otimes}_{\mathbf{k}} \mathcal{D}(T \times \bar{X})
$$

is an $\mathcal{O}$-linear topological identification. Finally, we have the $\mathcal{O}$-linear topological identification

$$
\widehat{1}_{\mathcal{O} \otimes \mathcal{D}(\bar{X})}^{-1}: \mathcal{O} \widehat{\otimes} \mathcal{D}(\bar{X}) \longrightarrow \mathcal{O} \widehat{\otimes}_{\iota} \mathcal{D}(\bar{X})
$$

from Remark 7.1.

Setting $\gamma^{\mathbf{k}}:=h_{*}^{\mathbf{k}} \circ\left(1_{\mathcal{O}} \widehat{\otimes}_{\mathbf{k}} \mathbf{P}\right) \circ \widehat{1}_{\mathcal{O} \otimes \mathcal{D}(\bar{X})}^{-1}$ we now consider the following diagram:

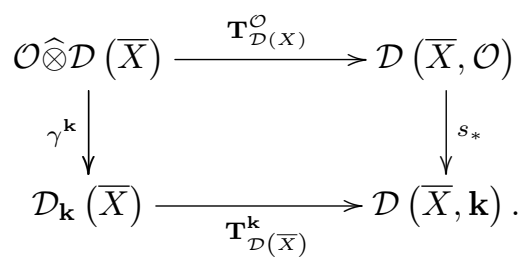

Since all the morphisms appearing in the diagram other than $\mathbf{T}_{\mathcal{D}(X)}^{\mathbf{k}}$ are $\mathcal{O}$-linear, topological identifications and $\mathbf{T}_{\mathcal{D}(X)}^{\mathbf{k}}$ is $(\mathcal{O}, \Sigma)$-equivariant, suffices to prove that the diagram is commutative. Indeed, since the arrows are $\mathcal{O}$-linear ftinuous, by (52) it suffices to show that $\mathbf{T}_{\mathcal{D}(X)}^{\mathbf{k}}\left(\gamma^{\mathbf{k}}\left(1 \widehat{\otimes} \delta_{\bar{x}}\right)\right)=s_{*}\left(\mathbf{T}_{\mathcal{D}(\bar{X})}^{\mathcal{O}}\left(1 \widehat{\otimes} \delta_{\bar{x}}\right)\right)$ for every $\bar{x} \in \bar{X}$. We have $1_{\mathcal{D}(T)}=\delta_{1}$, so that $\widehat{1}_{\mathcal{O} \otimes \mathcal{D}(\bar{X})}^{-1}\left(1 \widehat{\otimes} \delta_{\bar{x}}\right)=1 \widehat{\otimes}_{\iota} \delta_{\bar{x}}=1 \widehat{\otimes}_{\mathbf{k}} \delta_{1} \widehat{\otimes}_{\iota} \delta_{\bar{x}}$, and we see from (51), (58) and (50) that we have

$$
\begin{aligned}
\gamma^{\mathbf{k}}\left(1 \widehat{\otimes} \delta_{\bar{x}}\right) & =h_{*}^{\mathbf{k}}\left(\left(1_{\mathcal{O}} \widehat{\otimes}_{\mathbf{k}} \mathbf{P}\right)\left(1 \widehat{\otimes}_{\mathbf{k}} \delta_{1} \widehat{\otimes}_{\iota} \delta_{\bar{x}}\right)\right)=h_{*}^{\mathbf{k}}\left(1 \widehat{\otimes}_{\mathbf{k}} \delta_{(1, \bar{x})}\right)=1 \widehat{\otimes}_{\mathbf{k}} h_{*}\left(\delta_{(1, \bar{x})}\right) \\
& =1 \widehat{\otimes}_{\mathbf{k}} \delta_{h(1, \bar{x})}=1 \widehat{\otimes}_{\mathbf{k}} \delta_{s(\bar{x})} .
\end{aligned}
$$

It follows from (55) and (51) that

$$
\mathbf{T}_{\mathcal{D}(X)}^{\mathbf{k}}\left(\gamma^{\mathbf{k}}\left(1 \widehat{\otimes} \delta_{\bar{x}}\right)\right)=\delta_{s(\bar{x}) \mid \mathcal{A}_{\mathbf{k}}(X)}^{\mathcal{O}}=: \delta_{s(\bar{x})}^{\mathbf{k}} .
$$

On the other hand, $\mathbf{T}_{\mathcal{D}(\bar{X})}^{\mathcal{O}}\left(1 \widehat{\otimes} \delta_{\bar{x}}\right)=\delta_{\bar{x}}^{\mathcal{O}}$ by $(51)$, so that

$$
s_{*}\left(\mathbf{T}_{\mathcal{D}(\bar{X})}^{\mathcal{O}}\left(1 \widehat{\otimes} \delta_{\bar{x}}\right)\right)(F)=s_{*}\left(\delta_{\bar{x}}^{\mathcal{O}}\right)(F)=\delta_{\bar{x}}^{\mathcal{O}}\left(s^{*}(F)\right)=F(s(\bar{x}))=\delta_{s(\bar{x})}^{\mathbf{k}}(F)
$$

for every $F \in \mathcal{A}_{\mathbf{k}}(X)$. Hence $s_{*}\left(\mathbf{T}_{\mathcal{D}(\bar{X})}^{\mathcal{O}}\left(1 \widehat{\otimes} \delta_{\bar{x}}\right)\right)=\delta_{s(\bar{x})}^{\mathbf{k}}$ and the claim follows.

The following result is a formal generalization of [3, Theorem 3.7.4]. If $\mathbf{k}_{i} \in \operatorname{Hom}_{\mathcal{L}}\left(\mathcal{D}(T), \mathcal{O}_{i}\right)$ we write $\mathbf{k}_{1} \stackrel{\varphi}{\rightarrow} \mathbf{k}_{2}$ to mean that $\mathbf{k}_{2}=\varphi \circ \mathbf{k}_{1}$ for some $\varphi \in \operatorname{Hom}_{\mathcal{L}}\left(\mathcal{O}_{1}, \mathcal{O}_{2}\right)$ and set $I_{\varphi}:=\operatorname{ker}(\varphi)$ (with the subspace topology). There is an induced specialization map

$$
\varphi_{*}: \mathcal{D}_{\mathbf{k}_{1}}(X) \longrightarrow \mathcal{D}_{\mathbf{k}_{2}}(X), \quad \varphi_{*}\left(\alpha \widehat{\otimes}_{\mathbf{k}_{1}} \mu\right)=\varphi(\alpha) \widehat{\otimes}_{\mathbf{k}_{2}} \mu .
$$

It is easy to see that, viewing $\mathcal{O}_{i}$ as a $\mathcal{D}(T)$-module via $\mathbf{k}_{i}$, the map $\varphi: \mathcal{O}_{1} \rightarrow \mathcal{O}_{2}$ is $\mathcal{D}(T)$-bilinear. In particular, $I_{\varphi}$ is a $\mathcal{D}(T)$-sub-bimodule via $\mathbf{k}_{1}: \mathcal{D}(T) \rightarrow \mathcal{O}_{1}$ and we may form $I_{\varphi} \widehat{\otimes}_{\mathbf{k}_{1}} \mathcal{D}(X)$, which is a (left) $\mathcal{O}_{1}$-module and a (left) $\mathcal{D}(T)$-module (via $\mathbf{k}_{1}$ ). The notion of topological left exactness (resp. exactness) which appears in the statement of the following theorem is recalled just below it for the convenience of the reader. 
Theorem 7.7. Suppose $\mathbf{k}_{1} \stackrel{\varphi}{\rightarrow} \mathbf{k}_{2}$. Then the specialization sequence

$$
0 \longrightarrow I_{\varphi} \widehat{\otimes}_{\mathbf{k}_{1}} \mathcal{D}(X) \longrightarrow \mathcal{D}_{\mathbf{k}_{1}}(X) \stackrel{\varphi_{*}}{\longrightarrow} \mathcal{D}_{\mathbf{k}_{2}}(X)
$$

is topologically left exact. Furthermore, if $\varphi$ is surjective, then $\varphi_{*}$ is surjective and the resulting (62) is topologically exact.

Proof. Recall the $\mathcal{D}(T)$-linear, topological identifications $\gamma^{\mathbf{k}_{i}}: \mathcal{O}_{i} \widehat{\otimes} \mathcal{D}(\bar{X}) \rightarrow \mathcal{D}_{\mathbf{k}_{i}}(X)$ introduced before (59). Consider the following diagram

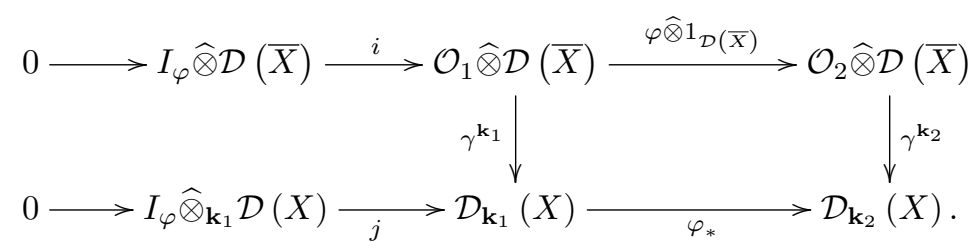

Let us assume the following facts, whose validity we be established below: $(i)$ there is a morphism $\gamma_{\mid}^{\mathbf{k}_{1}}: I_{\varphi} \widehat{\otimes} \mathcal{D}(\bar{X}) \rightarrow I_{\varphi} \widehat{\otimes}_{\mathbf{k}_{1}} \mathcal{D}(X)$ such the resulting square is commutative and with dense image, (ii) the square on the right is commutative, and (iii) the first row is topologically left exact (resp. exact when $\varphi$ is surjective). We claim that, given these facts, the continuous morphism $\gamma_{\mid}^{\mathbf{k}_{1}}: I_{\varphi} \widehat{\otimes} \mathcal{D}(\bar{X}) \rightarrow I_{\varphi} \widehat{\otimes}_{\mathbf{k}_{1}} \mathcal{D}(X)$ is a topological isomorphism; hence the topological left exactness (resp. exactness) of (62) (resp. of (62) when $\varphi$ is surjective) will follow from the corresponding exactness property of the first line. To prove the claim, we first remark that, because $\gamma^{\mathbf{k}_{1}} \circ i$ is injective, $\gamma_{\mid}^{\mathbf{k}_{1}}$ is too. Suppose now that $\left(\gamma_{\mid}^{\mathbf{k}_{1}}\left(x_{i}\right)\right) \rightarrow y$ is a net in $I_{\varphi} \widehat{\otimes}_{\mathbf{k}_{1}} \mathcal{D}(X)$ with $x_{i} \in I_{\varphi} \widehat{\otimes} \mathcal{D}(\bar{X})$. Because $j$ is continuous, we have $\left(\gamma^{\mathbf{k}_{1}}\left(i\left(x_{i}\right)\right)\right)=\left(j\left(\gamma_{\mid}^{\mathbf{k}_{1}}\left(x_{i}\right)\right)\right) \rightarrow j(y)$. Hence $\left(\gamma^{\mathbf{k}_{1}}\left(i\left(x_{i}\right)\right)\right)$ is a convergent net and, because $\gamma^{\mathbf{k}_{1}} \circ i$ is a topological subspace, $\left(x_{i}\right)$ is a Cauchy net and we have, $\left(x_{i}\right) \rightarrow x \in I_{\varphi} \widehat{\otimes} \mathcal{D}(\bar{X})$ by completeness of $I_{\varphi} \widehat{\otimes} \mathcal{D}(\bar{X})$. But then the continuity of $\gamma_{\mid}^{\mathbf{k}_{1}}$ implies that $\left(\gamma_{\mid}^{\mathbf{k}_{1}}\left(x_{i}\right)\right) \rightarrow \gamma_{\mid}^{\mathbf{k}_{1}}(x)$ and, hence, $y=\gamma_{\mid}^{\mathbf{k}_{1}}(x)$, because $I_{\varphi} \widehat{\otimes}_{\mathbf{k}_{1}} \mathcal{D}(X)$ is separated. This proves that the image of $\gamma_{\mid}^{\mathbf{k}_{1}}$ is closed and, because it is dense, we conclude that $\gamma_{\mid}^{\mathbf{k}_{1}}$ is surjective. Hence $\gamma_{\mid}^{\mathbf{k}_{1}}$ is a continuous bijection and the above argument proving the surjectivity of $\gamma_{\mid}^{\mathbf{k}_{1}}$ shows that, if $\left(\gamma_{\mid}^{\mathbf{k}_{1}}\left(x_{i}\right)\right) \rightarrow \gamma_{\mid}^{\mathbf{k}_{1}}(x)$, then $\left(x_{i}\right) \rightarrow x$ (because it proves the convergence $\left(x_{i}\right) \rightarrow x^{\prime} \in I_{\varphi} \widehat{\otimes} \mathcal{D}(\bar{X})$ and then, by continuity of $\gamma_{\mid}^{\mathbf{k}_{1}}, \gamma_{\mid}^{\mathbf{k}_{1}}(x)=\gamma_{\mid}^{\mathbf{k}_{1}}\left(x^{\prime}\right)$ implying $\left.x=x^{\prime}\right)$. We deduce that $\gamma_{1}^{\mathbf{k}_{1}}$ is a topological isomorphism, as desired.

Proof of $(i)$. Let us consider the following morphism, where the first arrow is $\mu \mapsto 1 \widehat{\otimes}_{\iota} \mu=\delta_{1} \widehat{\otimes}_{\iota} \mu$ :

$$
\beta: \mathcal{D}(\bar{X}) \longrightarrow \mathcal{D}(T) \widehat{\otimes}_{\iota} \mathcal{D}(\bar{X}) \stackrel{\mathbf{P}_{\mathcal{D}(T), \mathcal{D}(\bar{X})}^{K, K}}{\longrightarrow} \mathcal{D}(T \times \bar{X}) \stackrel{h_{*}}{\longrightarrow} \mathcal{D}(X) .
$$

It follows from $(51),(50)$ and the definition of $h$ that we have $\beta\left(\delta_{\bar{x}}\right)=\delta_{s(\bar{x})}$. Hence, setting

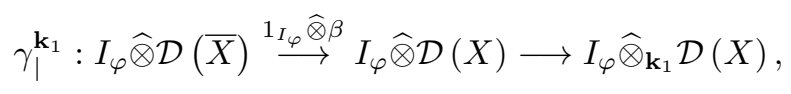

we see that $\gamma_{\mid}^{\mathbf{k}_{1}}\left(\alpha \widehat{\otimes} \delta_{\bar{x}}\right)=\alpha \widehat{\otimes}_{\mathbf{k}_{1}} \delta_{s(\bar{x})}$. It follows from (60) and the fact that $\gamma^{\mathbf{k}_{1}}$ is $\mathcal{O}_{1}$-linear that we also have $\gamma^{\mathbf{k}_{1}}\left(\alpha \widehat{\otimes} \delta_{\bar{x}}\right)=\alpha \widehat{\otimes}_{\mathbf{k}_{1}} \delta_{s(\bar{x})}$, proving that $\gamma_{\mid}^{\mathbf{k}_{1}}$ makes the diagram commutative (by density of the $K$-linear span of the elements $\alpha \widehat{\otimes} \delta_{\bar{x}}$ with $\left.\alpha \in I_{\varphi}\right)$. Since the $K$-linear span of the elements of the form $\alpha \widehat{\otimes}_{\mathbf{k}_{1}} \delta_{x}$ is dense in $I_{\varphi} \widehat{\otimes}_{\mathbf{k}_{1}} \mathcal{D}(X)$ (by (52)), suffices to prove that $\alpha \widehat{\otimes}_{\mathbf{k}_{1}} \delta_{x}$ with $\alpha \in I_{\varphi}$ and $x \in X$ belongs to the image of $\gamma_{\mid}^{\mathbf{k}_{1}}$ in order to deduce that $\gamma^{\mathbf{k}_{1}}$ has dense image. But because $h$ is surjective, we may write $x=t s(\bar{x})$ and then, by (54), we see that

$$
\begin{aligned}
\alpha \widehat{\otimes}_{\mathbf{k}_{1}} \delta_{x} & =\alpha \widehat{\otimes}_{\mathbf{k}_{1}} \delta_{t} \delta_{s(\bar{x})}=\alpha \mathbf{k}_{1}\left(\delta_{t}\right) \widehat{\otimes}_{\mathbf{k}_{1}} \delta_{s(\bar{x})} \\
& \left.=\gamma_{l}^{\mathbf{k}_{1}}\left(\alpha \mathbf{k}_{1}\left(\delta_{t}\right) \widehat{\otimes} \delta_{\bar{x}}\right) \in \operatorname{Im}\left(\gamma_{\mid}^{\mathbf{k}_{1}}\right) \text { (because } \alpha \mathbf{k}_{1}\left(\delta_{t}\right) \in I_{\varphi}\right) .
\end{aligned}
$$

Proof of (ii). In order to prove the commutativity of the right square, since the arrows are $\mathcal{D}(T)$-linear and continuous, by (52) it suffices to show that $\varphi_{*}\left(\gamma^{\mathbf{k}_{1}}\left(1 \widehat{\otimes} \delta_{\bar{x}}\right)\right)=\gamma^{\mathbf{k}_{2}}\left(\left(\varphi \widehat{\otimes} 1_{\mathcal{D}(\bar{X})}\right)\left(1 \widehat{\otimes} \delta_{\bar{x}}\right)\right)$. We have 
$\left(\varphi \widehat{\otimes} 1_{\mathcal{D}(\bar{X})}\right)\left(1 \widehat{\otimes} \delta_{\bar{x}}\right)=\varphi(1) \widehat{\otimes} \delta_{\bar{x}}=1 \widehat{\otimes} \delta_{\bar{x}}$ and, by $(60), \gamma^{\mathbf{k}_{i}}\left(1 \widehat{\otimes} \delta_{\bar{x}}\right)=1 \widehat{\otimes}_{\mathbf{k}_{i}} \delta_{s(\bar{x})}$. Hence, $\gamma^{\mathbf{k}_{2}}\left(\left(\varphi \widehat{\otimes} 1_{\mathcal{D}(\bar{X})}\right)\left(1 \widehat{\otimes} \delta_{\bar{x}}\right)\right)=$ $1 \widehat{\otimes}_{\mathbf{k}_{2}} \delta_{s(\bar{x})}$ and, by $(61)$,

$$
\varphi_{*}\left(\gamma^{\mathbf{k}_{1}}\left(1 \widehat{\otimes} \delta_{\bar{x}}\right)\right)=\varphi_{*}\left(1 \widehat{\otimes}_{\mathbf{k}_{1}} \delta_{s(\bar{x})}\right)=\varphi(1) \widehat{\otimes}_{\mathbf{k}_{2}} \delta_{s(\bar{x})}=1 \widehat{\otimes}_{\mathbf{k}_{2}} \delta_{s(\bar{x})} .
$$

Proof of (iii). The fact that the first line is topologically left exact (resp. exact when $\varphi$ is surjective) is a consequence of Lemma 7.8 below as follows. Consider the topologically left exact sequence of Banach spaces

$$
0 \longrightarrow I_{\varphi} \longrightarrow \mathcal{O}_{1} \stackrel{\varphi}{\longrightarrow} \mathcal{O}_{2} \text {. }
$$

We remark that, when $\varphi$ is surjective, it is a topological quotient by the open mapping theorem (see [38, Proposition 8.6]) and, hence, the above sequence is also topologically right exact. Also, by strictly paracompactness, $W=\mathcal{D}(\bar{X})$ is a topological direct sum of nuclear Fréchet spaces. Now apply the lemma.

Suppose that we are given a sequence

$$
0 \longrightarrow V_{1} \stackrel{\iota}{\longrightarrow} V_{2} \stackrel{\pi}{\longrightarrow} V_{3} \longrightarrow 0
$$

of morphisms of locally convex spaces. We give $0 \rightarrow \operatorname{ker}(\pi) \rightarrow V_{2}$ (resp. $V \rightarrow \operatorname{Im}(f) \rightarrow 0$ ) the initial topology (resp. final), i.e. the subspace topology (resp. the quotient topology). If $\pi \circ \iota=0$, then $\operatorname{Im}(\iota) \subset \operatorname{ker}(\pi)$ is always a continuous inclusion. We say that (63) is topologically exact at $V_{2}$ if it is algebraically exact and $\operatorname{Im}(\iota)=\operatorname{ker}(\pi)$ is a topological identification. We say that (63) is topologically left exact (resp. exact) if it is topologically exact at $V_{1}$ and $V_{2}$ (resp. and also at $V_{3}$ ). It is not difficult to see that (63) is topologically left exact if and only if it is algebraically left exact and $\iota$ is a topological subspace and that (63) is topologically exact if and only if it is algebraically exact, $\iota$ is a topological subspace and $\pi$ is a quotient space.

Lemma 7.8. The following facts hold:

(1) If (63) is topologically left exact, then the spaces $V_{k}$ for $k=1,2,3$ are Banach spaces, and $W=\bigoplus W_{i}$ is the sum of the nuclear Fréchet spaces $W_{i}$, then (63) $\widehat{\otimes} W$ is topologically left exact.

(2) If (63) is topologically exact, then the space $V_{2}$ is seminormed, and $W=\bigoplus W_{i}$ is the sum of semimetrizable spaces $W_{i}$, then (63) $\widehat{\otimes} W$ is topologically exact.

Proof. It is not true that the topological direct sum commutes with $V \widehat{\otimes}-$ in general; however, the reader may verify that this is true when $V$ is seminormed. It follows that $V_{k} \widehat{\otimes} W=\bigoplus_{i} V_{k} \widehat{\otimes} W_{i}$ for $k=1,2,3$ and, hence, we may assume that $W$ is a nuclear Fréchet (resp. semimetrizable) space in the proof of (1) (resp. $(2))$.

(2) We leave to the reader the verification that $-\otimes W$ is a topologically exact operation for every locally convex space $W$. We deduce that

$$
0 \longrightarrow V_{1} \otimes W \longrightarrow V_{2} \otimes W \longrightarrow V_{3} \otimes W \longrightarrow 0
$$

is a topologically exact sequence of seminormed spaces. Hence, the claim can be deduced from the more general assertion that, if

$$
0 \longrightarrow W_{1} \stackrel{\iota}{\longrightarrow} W_{2} \stackrel{\pi}{\longrightarrow} W_{3} \longrightarrow 0
$$

is a topologically exact sequence of semimetrizable spaces, then taking the completion preserves the topological exactness. Let $\mathcal{L}$ be a family of lattices which forms a basis at 0 for the topology of $W_{2}$ which is countable. Writing $\mathcal{O}_{K}$ for the ring of integers of $K$, we may form the following exact sequences of $\mathcal{O}_{K}$-modules

$$
0 \longrightarrow \frac{W_{1}}{\iota^{-1}(L)} \stackrel{\iota}{\longrightarrow} \frac{W_{2}}{L} \stackrel{\pi}{\longrightarrow} \frac{W_{3}}{\pi(L)} \longrightarrow 0 .
$$

Because $\left\{\frac{W_{1}}{\iota^{-1}(L)}\right\}_{L \in \mathcal{L}}$ is an inverse system with surjective transition maps and $\mathcal{L}$ is countable and directed, taking the inverse limit over $L \in \mathcal{L}$ yields the exact sequence

$$
0 \longrightarrow \lim _{\leftarrow L \in \mathcal{L}} \frac{W_{1}}{\iota^{-1}(L)} \stackrel{\iota}{\longrightarrow} \lim _{\leftarrow L \in \mathcal{L}} \frac{W_{2}}{L} \stackrel{\pi}{\longrightarrow} \lim _{\leftarrow L \in \mathcal{L}} \frac{W_{3}}{\pi(L)} \longrightarrow 0 .
$$

But because (64) is topologically exact, we know that $\left\{\iota^{-1}(L): L \in \mathcal{L}\right\}$ (resp. $\{\pi(L): L \in \mathcal{L}\}$ ) is a family of lattices that forms a basis at 0 for the topology of $W_{1}$ (resp. $W_{3}$ ). Hence, by the explicit construction of 
the completion $c: W \rightarrow \widehat{W}$ as an inverse limit (see, e.g., [38, proof of Proposition 7.5]), we have (65) $=\widehat{(64)}$, proving the exactness of $\widehat{(64)}$. The topological exactness follows from that of (64), using the fact that the association $L \mapsto \overline{c(L)}$ establishes a bijection between the sets of open lattices in the locally convex space $W$ and those in its completion $\widehat{W}$ (with inverse $\widehat{L} \mapsto c^{-1}(\widehat{L})$, see [38, proof of Proposition 7.5]).

(1) We can split the topologically left exact sequence (63) in the topologically exact sequence

$$
0 \longrightarrow V_{1} \longrightarrow V_{2} \longrightarrow \frac{V_{2}}{V_{1}} \longrightarrow 0
$$

and the continuous inclustion $\frac{V_{2}}{V_{1}} \rightarrow V_{3}$ of Banach spaces. Applying (2) we deduce that

$$
0 \longrightarrow V_{1} \widehat{\otimes} W \longrightarrow V_{2} \widehat{\otimes} W \longrightarrow \frac{V_{2}}{V_{1}} \widehat{\otimes} W \longrightarrow 0
$$

is topologically exact. The following result implies that $\frac{V_{2}}{V_{1}} \widehat{\otimes} W \rightarrow V_{3} \widehat{\otimes} W$ is again a continuous inclusion, proving our claim.

Sublemma. Suppose that $U_{1} \rightarrow U_{2}$ is an injective morphism of Hausdorff and complete locally convex spaces and that $W$ is a nuclear Fréchet space. Then $U_{1} \widehat{\otimes} W \rightarrow U_{2} \widehat{\otimes} W$ is again an injective morphism.

Proof of the sublemma. Because $W$ is a nuclear Fréchet space, $\mathcal{L}(W, K)$ is of compact type (see [38, Proposition 16.5]). But because it is an inductive limit of Banach spaces, $\mathcal{L}(W, K)$ is bornological (see [38, pag. 39 Example 2]) and, by [38, Proposition 16.10], it is reflexive. Since $\mathcal{L}(W, K)$ is bornological and reflexive, $\mathcal{L}(\mathcal{L}(W, K), U)$ is (Hausdorff and) complete for every Hausdorff and complete locally convex space $U$ (see [38, Proposition 7.16]). We can now apply [38, Proposition 18.2] to deduce that there is a canonical isomorphism

$$
\mathcal{L}(\mathcal{L}(W, K), K) \widehat{\otimes} U \stackrel{\sim}{\longrightarrow} \mathcal{C C}(\mathcal{L}(W, K), U),
$$

with $\mathcal{C C} \subset \mathcal{L}$ the closure of the space of maps having finite image. Applying the reflexivity of $W$ (see [38, Proposition 16.5]), we deduce the canonical identification

$$
W \widehat{\otimes} U \stackrel{\sim}{\longrightarrow} \mathcal{C C}(\mathcal{L}(W, K), U) .
$$

But it is clear that $\mathcal{C C}(\mathcal{L}(W, K),-)$ preserves injections, proving the claim.

7.2. Derivatives of distributions. Suppose that $\mathbf{k}_{1} \stackrel{\varphi}{\rightarrow} \mathbf{k}_{2}$ with $\mathbf{k}_{i} \in \operatorname{Hom}_{\mathcal{L}}\left(\mathcal{D}(T), \mathcal{O}_{i}\right)$. We write $\operatorname{Der}(\varphi)$ to denote the space of morphisms of locally convex spaces $\partial: \mathcal{O}_{1} \rightarrow \mathcal{O}_{2}$ such that

$$
\partial(\alpha \beta)=\partial(\alpha) \varphi(\beta)+\varphi(\alpha) \partial(\beta) .
$$

We may therefore consider the following morphism of locally convex spaces:

$$
\partial_{\mathcal{D}(X)}: I_{\varphi} \widehat{\otimes} \mathcal{D}(X) \longrightarrow \mathcal{O}_{1} \widehat{\otimes} \mathcal{D}(X) \stackrel{\partial \widehat{\otimes} 1_{\mathcal{D}(X)}}{\longrightarrow} \mathcal{O}_{2} \widehat{\otimes} \mathcal{D}(X) .
$$

Note that $\partial_{\mathcal{D}(X)}$ is left $\mathcal{D}(T)$-linear:

$$
\begin{aligned}
\partial_{\mathcal{D}(X)}\left(\mathbf{k}_{1}(\nu) \alpha \widehat{\otimes} \mu\right) & =\partial\left(\mathbf{k}_{1}(\nu) \alpha\right) \widehat{\otimes} \mu=\partial\left(\mathbf{k}_{1}(\nu)\right) \varphi(\alpha) \widehat{\otimes} \mu+\varphi\left(\mathbf{k}_{1}(\nu)\right) \partial(\alpha) \widehat{\otimes} \mu \\
& =\mathbf{k}_{2}(\nu) \partial(\alpha) \widehat{\otimes} \mu=\mathbf{k}_{2}(\nu) \partial_{\mathcal{D}(X)}(\alpha \widehat{\otimes} \mu)
\end{aligned}
$$

for every $\nu \in \mathcal{D}(T), \alpha \in I_{\varphi}$ and $\mu \in \mathcal{D}(X)$. Recall that $X$ is endowed with a right action of a semigroup $\Sigma$ compatible with the $T$-action. Then $\partial_{\mathcal{D}(X)}$ is $\Sigma$-equivariant, being the composition of $\Sigma$-equivariant maps. The proof of the following result, left to the reader, is an easy consequence of the fact that $\partial(\alpha \beta)=\partial(\alpha) \varphi(\beta)$ when $\alpha \in I_{\varphi}$ and $\beta \in \mathcal{O}_{1}$.

Lemma 7.9. If $\mathbf{k}_{1} \stackrel{\varphi}{\rightarrow} \mathbf{k}_{2}$ and $\partial \in \operatorname{Der}(\varphi)$, then there is a unique morphism of locally convex spaces $\partial_{\varphi}=\partial_{\varphi, \mathcal{D}(X)}$ making the following diagram commutative, which is $(\mathcal{D}(T), \Sigma)$-equivariant:

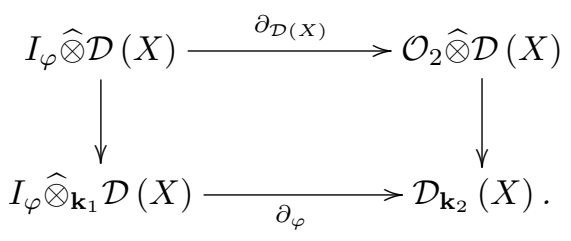


Until the end of this subsection, assume that $\pi: X \rightarrow \bar{X}$ is a trivial $T$-bundle. Let $\mathbf{k}_{1} \stackrel{\varphi}{\rightarrow} \mathbf{k}_{2}$ and let $\partial \in \operatorname{Der}(\varphi)$. Then we may define

$$
\partial_{\varphi, s}: \mathcal{D}_{\mathbf{k}_{1}}(X) \longrightarrow \mathcal{D}_{\mathbf{k}_{2}}(X)
$$

by means of the following commutative diagram

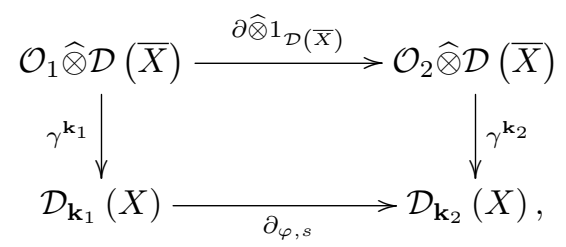

where we recall that we have

$$
\gamma^{\mathbf{k}_{i}}=\gamma_{s}^{\mathbf{k}_{i}}: \mathcal{O}_{i} \widehat{\otimes} \mathcal{D}(\bar{X}) \stackrel{\mathbf{T}_{\mathcal{D}(\overline{\mathcal{O}})}}{\longrightarrow} \mathcal{D}\left(\bar{X}, \mathcal{O}_{i}\right) \stackrel{s_{*}^{\mathbf{k}_{i}}}{\longrightarrow} \mathcal{D}\left(X, \mathbf{k}_{i}\right) \stackrel{\left(\mathbf{T}_{\mathcal{D}(X)}^{\mathbf{k}_{i}}\right)^{-1}}{\longrightarrow} \mathcal{D}_{\mathbf{k}_{i}}(X)
$$

We define

$$
\partial_{\varphi, s}: \mathcal{A}_{\mathbf{k}_{1}}(X) \longrightarrow \mathcal{A}_{\mathbf{k}_{2}}(X)
$$

by means of the following commutative diagram

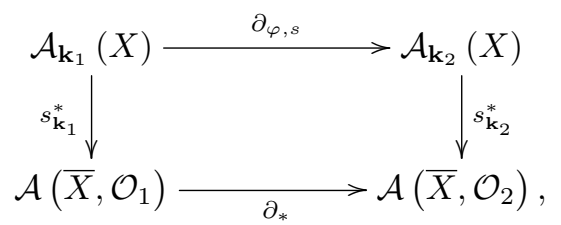

where $\partial_{*}(f):=\partial \circ f$.

Proposition 7.10. If $\mu \in \mathcal{D}_{\mathbf{k}_{1}}(X)$ and $F \in \mathcal{A}_{\mathbf{k}_{1}}(X)$, we have the formula

$$
\partial\left(\mathbf{T}_{\mathcal{D}(X)}^{\mathbf{k}_{1}}(\mu)(F)\right)=\mathbf{T}_{\mathcal{D}(X)}^{\mathbf{k}_{2}}\left(\partial_{\varphi, s}(\mu)\right)\left(\varphi_{*}(F)\right)+\mathbf{T}_{\mathcal{D}(X)}^{\mathbf{k}_{2}}\left(\varphi_{*}(\mu)\right)\left(\partial_{\varphi, s}(F)\right),
$$

where $\varphi_{*}(F):=\varphi \circ F$.

Proof. If $\alpha \in \mathcal{O}_{1}, \bar{x} \in \bar{X}$ and $f \in \mathcal{A}\left(\bar{X}, \mathcal{O}_{1}\right)$, by (51) we have, setting $\partial_{\mathcal{D}(\bar{X})}:=\partial \widehat{\otimes} 1_{\mathcal{D}(\bar{X})}, \varphi_{\mathcal{D}(\bar{X})}:=$ $\varphi \widehat{\otimes} 1_{\mathcal{D}(\bar{X})}$ and $\varphi_{*}(f):=\partial \circ f$

$$
\begin{array}{ll}
\partial\left(\mathbf{T}_{\mathcal{D}(\bar{X})}^{\mathcal{O}_{1}}\left(\alpha \widehat{\otimes} \delta_{\bar{x}}\right)(f)\right) & \partial\left(\alpha \delta_{\bar{x}}^{\mathcal{O}_{1}}(f)\right)=\partial(\alpha) \varphi\left(\delta_{\bar{x}}^{\mathcal{O}_{1}}(f)\right)+\varphi(\alpha) \partial\left(\delta_{\bar{x}}^{\mathcal{O}_{1}}(f)\right) \\
& =\partial(\alpha) \varphi(f(\bar{x}))+\varphi(\alpha) \partial(f(\bar{x})), \\
\mathbf{T}_{\mathcal{D}(\bar{X})}^{\mathcal{O}_{2}}\left(\partial_{\mathcal{D}(\bar{X})}\left(\alpha \widehat{\otimes} \delta_{\bar{x}}\right)\right)\left(\varphi_{*}(f)\right) & =\mathbf{T}_{\mathcal{D}(\bar{X})}^{\mathcal{O}_{2}}\left(\partial(\alpha) \widehat{\otimes} \delta_{\bar{x}}\right)\left(\varphi_{*}(f)\right)=\partial(\alpha) \delta_{\bar{x}}^{\mathcal{O}_{2}}\left(\varphi_{*}(f)\right)=\partial(\alpha) \varphi(f(\bar{x})) \\
\mathbf{T}_{\mathcal{D}(\bar{X})}^{\mathcal{O}_{2}}\left(\varphi_{\mathcal{D}(\bar{X})}\left(\alpha \widehat{\otimes} \delta_{\bar{x}}\right)\right)\left(\partial_{*}(f)\right)= & \mathbf{T}_{\mathcal{D}(\bar{X})}^{\mathcal{O}_{2}}\left(\varphi(\alpha) \widehat{\otimes} \delta_{\bar{x}}\right)\left(\partial_{*}(f)\right)=\varphi(\alpha) \delta_{\bar{x}}^{\mathcal{O}_{2}}\left(\partial_{*}(f)\right)=\varphi(\alpha) \partial(f(\bar{x})) .
\end{array}
$$

By $K$-linearity, continuity and density of the span of the Dirac distributions we deduce

$$
\partial\left(\mathbf{T}_{\mathcal{D}(\bar{X})}^{\mathcal{O}_{1}}(\bar{\mu})(f)\right)=\mathbf{T}_{\mathcal{D}(\bar{X})}^{\mathcal{O}_{2}}\left(\partial_{\mathcal{D}(\bar{X})}(\bar{\mu})\right)\left(\varphi_{*}(f)\right)+\mathbf{T}_{\mathcal{D}(\bar{X})}^{\mathcal{O}_{2}}\left(\varphi_{\mathcal{D}(\bar{X})}(\bar{\mu})\right)\left(\partial_{*}(f)\right), \bar{\mu} \in \mathcal{O}_{1} \widehat{\otimes} \mathcal{D}(\bar{X})
$$

In particular, with $f=s_{\mathbf{k}_{1}}^{*}(F)$ and $\bar{\mu}=\left(\gamma^{\mathbf{k}_{1}}\right)^{-1}(\mu)$, we deduce

$$
\begin{aligned}
\partial\left(\mathbf{T}_{\mathcal{D}(\bar{X})}^{\mathcal{O}_{1}}\left(\left(\gamma^{\mathbf{k}_{1}}\right)^{-1}(\mu)\right)\left(s_{\mathbf{k}_{1}}^{*}(F)\right)\right)= & \mathbf{T}_{\mathcal{D}(\bar{X})}^{\mathcal{O}_{2}}\left(\partial_{\mathcal{D}(\bar{X})}\left(\left(\gamma^{\mathbf{k}_{1}}\right)^{-1}(\mu)\right)\right)\left(\varphi_{*}\left(s_{\mathbf{k}_{1}}^{*}(F)\right)\right) \\
& +\mathbf{T}_{\mathcal{D}(\bar{X})}^{\mathcal{O}_{2}}\left(\varphi_{\mathcal{D}(\bar{X})}\left(\left(\gamma^{\mathbf{k}_{1}}\right)^{-1}(\mu)\right)\right)\left(\partial_{*}\left(s_{\mathbf{k}_{1}}^{*}(F)\right)\right) . \\
& 25
\end{aligned}
$$


By the commutative diagram $(59), \mathbf{T}_{\mathcal{D}(\bar{X})}^{\mathcal{O}_{1}} \circ\left(\gamma^{\mathbf{k}_{1}}\right)^{-1}=\left(s_{*}^{\mathbf{k}_{1}}\right)^{-1} \circ \mathbf{T}_{\mathcal{D}(X)}^{\mathbf{k}_{1}}$, so that $\mathbf{T}_{\mathcal{D}(\bar{X})}^{\mathcal{O}_{1}}\left(\left(\gamma^{\mathbf{k}_{1}}\right)^{-1}(\mu)\right)=$ $\left(s_{*}^{\mathbf{k}_{1}}\right)^{-1}\left(\mathbf{T}_{\mathcal{D}(X)}^{\mathbf{k}_{1}}(\mu)\right)$ and, since $s_{*}^{\mathbf{k}_{1}}$ is the dual of $s_{\mathbf{k}_{1}}^{*}$,

$$
\mathbf{T}_{\mathcal{D}(\bar{X})}^{\mathcal{O}_{1}}\left(\left(\gamma^{\mathbf{k}_{1}}\right)^{-1}(\mu)\right)\left(s_{\mathbf{k}_{1}}^{*}(F)\right)=\mathbf{T}_{\mathcal{D}(X)}^{\mathbf{k}_{1}}(\mu)\left(\left(s_{\mathbf{k}_{1}}^{*}\right)^{-1} s_{\mathbf{k}_{1}}^{*}(F)\right)=\mathbf{T}_{\mathcal{D}(X)}^{\mathbf{k}_{1}}(\mu)(F) .
$$

We also have, by definition, $\partial_{\mathcal{D}(\bar{X})}\left(\left(\gamma^{\mathbf{k}_{1}}\right)^{-1}(\mu)\right)=\left(\gamma^{\mathbf{k}_{2}}\right)^{-1}\left(\partial_{\varphi, s}(\mu)\right)$ so that, again using $\mathbf{T}_{\mathcal{D}(\bar{X})}^{\mathcal{O}_{2}}{ }^{\circ}$ $\left(\gamma^{\mathbf{k}_{2}}\right)^{-1}=\left(s_{*}^{\mathbf{k}_{2}}\right)^{-1} \circ \mathbf{T}_{\mathcal{D}(X)}^{\mathbf{k}_{2}}$, we find $\mathbf{T}_{\mathcal{D}(\bar{X})}^{\mathcal{O}_{2}}\left(\partial_{\mathcal{D}(\bar{X})}\left(\left(\gamma^{\mathbf{k}_{1}}\right)^{-1}(\mu)\right)\right)=\left(s_{*}^{\mathbf{k}_{2}}\right)^{-1}\left(\mathbf{T}_{\mathcal{D}(X)}^{\mathbf{k}_{2}}\left(\partial_{\varphi, s}(\mu)\right)\right) ;$ since $s_{*}^{\mathbf{k}_{2}}$ is the dual of $s_{\mathbf{k}_{2}}^{*}$,

$$
\mathbf{T}_{\mathcal{D}(\bar{X})}^{\mathcal{O}_{2}}\left(\partial_{\mathcal{D}(\bar{X})}\left(\left(\gamma^{\mathbf{k}_{1}}\right)^{-1}(\mu)\right)\right)\left(\varphi_{*}\left(s_{\mathbf{k}_{1}}^{*}(F)\right)\right)=\mathbf{T}_{\mathcal{D}(X)}^{\mathbf{k}_{2}}\left(\partial_{\varphi, s}(\mu)\right)\left(\left(s_{\mathbf{k}_{2}}^{*}\right)^{-1}\left(\varphi_{*}\left(s_{\mathbf{k}_{1}}^{*}(F)\right)\right)\right) .
$$

We now remark that, as in the proof of Lemma 7.4, $r_{\mathbf{k}_{i}}=\left(s_{*, \mathbf{k}_{i}}\right)^{-1}$ with $r_{\mathbf{k}_{i}}(f)(h(t, \bar{x}))=\mathbf{k}_{i}\left(\delta_{t}\right) f(\bar{x})$, so that

$$
\begin{aligned}
r_{\mathbf{k}_{2}}\left(\varphi_{*}\left(s_{\mathbf{k}_{1}}^{*}(F)\right)\right)(h(t, \bar{x})) & =\mathbf{k}_{2}\left(\delta_{t}\right) \varphi_{*}\left(s_{\mathbf{k}_{1}}^{*}(F)\right)(\bar{x})=\varphi\left(\mathbf{k}_{1}\left(\delta_{t}\right)\right) \varphi\left(s_{\mathbf{k}_{1}}^{*}(F)(\bar{x})\right) \\
& =\varphi\left(\mathbf{k}_{1}\left(\delta_{t}\right) s_{\mathbf{k}_{1}}^{*}(F)(\bar{x})\right)=\varphi\left(r_{\mathbf{k}_{1}}\left(s_{\mathbf{k}_{1}}^{*}(F)\right)(h(t, \bar{x}))\right) \\
& =\varphi(F(h(t, \bar{x})))=\varphi_{*}(F)(h(t, \bar{x})) .
\end{aligned}
$$

It follows that $\left(s_{\mathbf{k}_{2}}^{*}\right)^{-1}\left(\varphi_{*}\left(s_{\mathbf{k}_{1}}^{*}(F)\right)\right)=\varphi_{*}(F)$ and we deduce

$$
\mathbf{T}_{\mathcal{D}(\bar{X})}^{\mathcal{O}_{2}}\left(\partial_{\mathcal{D}(\bar{X})}\left(\left(\gamma^{\mathbf{k}_{1}}\right)^{-1}(\mu)\right)\right)\left(\varphi_{*}\left(s_{\mathbf{k}_{1}}^{*}(F)\right)\right)=\mathbf{T}_{\mathcal{D}(X)}^{\mathbf{k}_{2}}\left(\partial_{\varphi, s}(\mu)\right)\left(\varphi_{*}(F)\right)
$$

We have, by definition, $\partial_{*}\left(s_{\mathbf{k}_{1}}^{*}(F)\right)=s_{\mathbf{k}_{2}}^{*}\left(\partial_{\varphi, s}(F)\right)$. Since $\mathbf{T}_{\mathcal{D}(\bar{X})}^{\mathcal{O}_{2}}=\left(s_{*}^{\mathbf{k}_{2}}\right)^{-1} \circ \mathbf{T}_{\mathcal{D}(X)}^{\mathbf{k}_{2}} \circ \gamma^{\mathbf{k}_{2}}$ and $s_{*}^{\mathbf{k}_{2}}$ is the dual of $s_{\mathbf{k}_{2}}^{*}$, we see that

$$
\begin{aligned}
& \mathbf{T}_{\mathcal{D}(\bar{X})}^{\mathcal{O}_{2}}\left(\varphi_{\mathcal{D}(\bar{X})}\left(\left(\gamma^{\mathbf{k}_{1}}\right)^{-1}(\mu)\right)\right)\left(\partial_{*}\left(s_{\mathbf{k}_{1}}^{*}(F)\right)\right)=\mathbf{T}_{\mathcal{D}(\bar{X})}^{\mathcal{O}_{2}}\left(\varphi_{\mathcal{D}(\bar{X})}\left(\left(\gamma^{\mathbf{k}_{1}}\right)^{-1}(\mu)\right)\right)\left(s_{\mathbf{k}_{2}}^{*}\left(\partial_{\varphi, s}(F)\right)\right) \\
& =\left(s_{*}^{\mathbf{k}_{2}}\right)^{-1}\left(\mathbf{T}_{\mathcal{D}(X)}^{\mathbf{k}_{2}}\left(\gamma^{\mathbf{k}_{2}}\left(\varphi_{\mathcal{D}(\bar{X})}\left(\left(\gamma^{\mathbf{k}_{1}}\right)^{-1}(\mu)\right)\right)\right)\right)\left(s_{\mathbf{k}_{2}}^{*}\left(\partial_{\varphi, s}(F)\right)\right) \\
& =\left(\mathbf{T}_{\mathcal{D}(X)}^{\mathbf{k}_{2}}\left(\gamma^{\mathbf{k}_{2}}\left(\varphi_{\mathcal{D}(\bar{X})}\left(\left(\gamma^{\mathbf{k}_{1}}\right)^{-1}(\mu)\right)\right)\right)\right)\left(\left(s_{\mathbf{k}_{2}}^{*}\right)^{-1}\left(s_{\mathbf{k}_{2}}^{*}\left(\partial_{\varphi, s}(F)\right)\right)\right) \\
& =\left(\mathbf{T}_{\mathcal{D}(X)}^{\mathbf{k}_{2}}\left(\gamma^{\mathbf{k}_{2}}\left(\varphi_{\mathcal{D}(\bar{X})}\left(\left(\gamma^{\mathbf{k}_{1}}\right)^{-1}(\mu)\right)\right)\right)\right)\left(\partial_{\varphi, s}(F)\right) .
\end{aligned}
$$

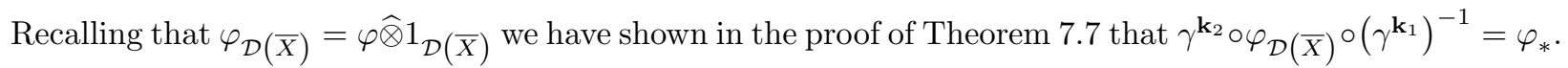
We deduce

$$
\mathbf{T}_{\mathcal{D}(\bar{X})}^{\mathcal{O}_{2}}\left(\varphi_{\mathcal{D}(\bar{X})}\left(\left(\gamma^{\mathbf{k}_{1}}\right)^{-1}(\mu)\right)\right)\left(\partial_{*}\left(s_{\mathbf{k}_{1}}^{*}(F)\right)\right)=\mathbf{T}_{\mathcal{D}(X)}^{\mathbf{k}_{2}}\left(\varphi_{*}(\mu)\right)\left(\partial_{\varphi, s}(F)\right) .
$$

The claim now follows from (66), (67), (68) and (69).

Proposition 7.11. If $\alpha \in \mathcal{O}_{1}$ and $\mu \in \mathcal{D}_{\mathbf{k}_{1}}(X)$, then

$$
\partial_{\varphi, s}(\alpha \mu)=\partial(\alpha) \varphi_{*}(\mu)+\varphi(\alpha) \partial_{\varphi, s}(\mu) .
$$

Proof. Suppose, first, that this formula holds when $\mu=1 \widehat{\otimes}_{\mathbf{k}_{1}} \delta_{x}$. Since $\varphi_{*}\left(\beta \widehat{\otimes}_{\mathbf{k}_{1}} \delta_{x}\right)=\varphi(\beta) \varphi_{*}\left(1 \widehat{\otimes}_{\mathbf{k}_{1}} \delta_{x}\right)$ by (61), we see that

$$
\begin{aligned}
\partial_{\varphi, s}\left(\alpha \cdot \beta \widehat{\otimes}_{\mathbf{k}_{1}} \delta_{x}\right) & =\partial_{\varphi, s}\left(\alpha \beta \cdot 1 \widehat{\otimes}_{\mathbf{k}_{1}} \delta_{x}\right)=\partial(\alpha \beta) \varphi_{*}\left(1 \widehat{\otimes}_{\mathbf{k}_{1}} \delta_{x}\right)+\varphi(\alpha \beta) \partial_{\varphi, s}\left(1 \widehat{\otimes}_{\mathbf{k}_{1}} \delta_{x}\right) \\
& =\partial(\alpha) \varphi(\beta) \varphi_{*}\left(1 \widehat{\otimes}_{\mathbf{k}_{1}} \delta_{x}\right)+\varphi(\alpha) \partial(\beta) \varphi_{*}\left(1 \widehat{\otimes}_{\mathbf{k}_{1}} \delta_{x}\right)+\varphi(\alpha) \varphi(\beta) \partial_{\varphi, s}\left(1 \widehat{\otimes}_{\mathbf{k}_{1}} \delta_{x}\right) \\
& =\partial(\alpha) \varphi_{*}\left(\beta \widehat{\otimes}_{\mathbf{k}_{1}} \delta_{x}\right)+\varphi(\alpha)\left(\partial(\beta) \varphi_{*}\left(1 \widehat{\otimes}_{\mathbf{k}_{1}} \delta_{x}\right)+\varphi(\beta) \partial_{\varphi, s}\left(1 \widehat{\otimes}_{\mathbf{k}_{1}} \delta_{x}\right)\right) \\
& =\partial(\alpha) \varphi_{*}\left(\beta \widehat{\otimes}_{\mathbf{k}_{1}} \delta_{x}\right)+\varphi(\alpha) \partial_{\varphi, s}\left(\beta \widehat{\otimes}_{\mathbf{k}_{1}} \delta_{x}\right) . \\
&
\end{aligned}
$$


Since the $K$-linear span of the elements $\alpha \widehat{\otimes}_{\mathbf{k}_{1}} \delta_{x}$ is dense in $\mathcal{D}_{\mathbf{k}_{1}}(X)$, the claim then follows by the $K$-linearity and continuity of the maps involved. We must therefore prove that

$$
\partial_{\varphi, s}\left(\alpha \widehat{\otimes}_{\mathbf{k}_{1}} \delta_{h(t, \bar{x})}\right)=\partial(\alpha) \varphi_{*}\left(1 \widehat{\otimes}_{\mathbf{k}_{1}} \delta_{h(t, \bar{x})}\right)+\varphi(\alpha) \partial_{\varphi, s}\left(1 \widehat{\otimes}_{\mathbf{k}_{1}} \delta_{h(t, \bar{x})}\right) .
$$

According to $(60), \gamma^{\mathbf{k}_{i}}\left(1 \widehat{\otimes} \delta_{\bar{x}}\right)=1 \widehat{\otimes}_{\mathbf{k}_{i}} \delta_{s(\bar{x})}$ and then, by $\mathcal{D}(T)$-linearity of $\gamma^{\mathbf{k}_{i}}$,

$$
\gamma^{\mathbf{k}_{i}}\left(\mathbf{k}_{i}\left(\delta_{t}\right) \widehat{\otimes} \delta_{\bar{x}}\right)=\mathbf{k}_{i}\left(\delta_{t}\right) \gamma^{\mathbf{k}_{i}}\left(1 \widehat{\otimes} \delta_{\bar{x}}\right)=\mathbf{k}_{i}\left(\delta_{t}\right) \widehat{\otimes}_{\mathbf{k}_{i}} \delta_{s(\bar{x})}=1 \widehat{\otimes}_{\mathbf{k}_{i}} \delta_{t} \cdot \delta_{s(\bar{x})}=1 \widehat{\otimes}_{\mathbf{k}_{i}} \delta_{h(t, \bar{x})},
$$

where $\delta_{t} \cdot \delta_{s(\bar{x})}=\delta_{t s(\bar{x})}=\delta_{h(t, \bar{x})}$ follows from (54) and the definition of $h$. We deduce $\gamma^{\mathbf{k}_{i}}\left(\alpha \mathbf{k}_{i}\left(\delta_{t}\right) \widehat{\otimes} \delta_{\bar{x}}\right)=$ $\alpha \widehat{\otimes}_{\mathbf{k}_{i}} \delta_{h(t, \bar{x})}$ by $\mathcal{O}_{i}$-linearity of $\gamma^{\mathbf{k}_{i}}$. It follows that

$$
\partial_{\varphi, s}\left(\alpha \widehat{\otimes}_{\mathbf{k}_{1}} \delta_{h(t, \bar{x})}\right)=\gamma^{\mathbf{k}_{2}}\left(\partial_{\mathcal{D}(\bar{X})}\left(\left(\gamma^{\mathbf{k}_{1}}\right)^{-1}\left(\alpha \widehat{\otimes}_{\mathbf{k}_{1}} \delta_{h(t, \bar{x})}\right)\right)\right)=\gamma^{\mathbf{k}_{2}}\left(\partial_{\mathcal{D}(\bar{X})}\left(\alpha \mathbf{k}_{1}\left(\delta_{t}\right) \widehat{\otimes} \delta_{\bar{x}}\right)\right) .
$$

We compute

$$
\begin{aligned}
\partial_{\mathcal{D}(\bar{X})}\left(\alpha \mathbf{k}_{1}\left(\delta_{t}\right) \widehat{\otimes} \delta_{\bar{x}}\right) & =\partial\left(\alpha \mathbf{k}_{1}\left(\delta_{t}\right)\right) \widehat{\otimes} \delta_{\bar{x}}=\partial(\alpha) \varphi\left(\mathbf{k}_{1}\left(\delta_{t}\right)\right) \widehat{\otimes} \delta_{\bar{x}}+\varphi(\alpha) \partial\left(\mathbf{k}_{1}\left(\delta_{t}\right)\right) \widehat{\otimes} \delta_{\bar{x}} \\
& =\partial(\alpha) \mathbf{k}_{2}\left(\delta_{t}\right) \widehat{\otimes} \delta_{\bar{x}}+\varphi(\alpha) \partial\left(\mathbf{k}_{1}\left(\delta_{t}\right)\right) \widehat{\otimes} \delta_{\bar{x}} \\
& =\left(\gamma^{\mathbf{k}_{2}}\right)^{-1}\left(\partial(\alpha) \widehat{\otimes}_{\mathbf{k}_{2}} \delta_{h(t, \bar{x})}\right)+\varphi(\alpha) \partial\left(\mathbf{k}_{1}\left(\delta_{t}\right)\right) \widehat{\otimes} \delta_{\bar{x}},
\end{aligned}
$$

implying that we have

$$
\partial_{\varphi, s}\left(\alpha \widehat{\otimes}_{\mathbf{k}_{1}} \delta_{h(t, \bar{x})}\right)=\partial(\alpha) \widehat{\otimes}_{\mathbf{k}_{2}} \delta_{h(t, \bar{x})}+\gamma^{\mathbf{k}_{2}}\left(\varphi(\alpha) \partial\left(\mathbf{k}_{1}\left(\delta_{t}\right)\right) \widehat{\otimes} \delta_{\bar{x}}\right) .
$$

Furthermore, setting $\alpha=1$, we see that

$$
\partial_{\varphi, s}\left(1 \widehat{\otimes}_{\mathbf{k}_{1}} \delta_{h(t, \bar{x})}\right)=\gamma^{\mathbf{k}_{2}}\left(\partial\left(\mathbf{k}_{1}\left(\delta_{t}\right)\right) \widehat{\otimes} \delta_{\bar{x}}\right)
$$

According to (61) $\varphi_{*}\left(1 \widehat{\otimes}_{\mathbf{k}_{1}} \delta_{h(t, \bar{x})}\right)=1 \widehat{\otimes}_{\mathbf{k}_{2}} \delta_{h(t, \bar{x})}$, so that

$$
\partial(\alpha) \widehat{\otimes}_{\mathbf{k}_{2}} \delta_{h(t, \bar{x})}=\partial(\alpha) \varphi_{*}\left(1 \widehat{\otimes}_{\mathbf{k}_{1}} \delta_{h(t, \bar{x})}\right) .
$$

On the other hand, by $\mathcal{O}_{2}$-linearity of $\gamma^{\mathbf{k}_{2}}, \gamma^{\mathbf{k}_{2}}\left(\varphi(\alpha) \partial\left(\mathbf{k}_{1}\left(\delta_{t}\right)\right) \widehat{\otimes} \delta_{\bar{x}}\right)=\varphi(\alpha) \gamma^{\mathbf{k}_{2}}\left(\partial\left(\mathbf{k}_{1}\left(\delta_{t}\right)\right) \widehat{\otimes} \delta_{\bar{x}}\right)$ so that (72) gives

$$
\gamma^{\mathbf{k}_{2}}\left(\varphi(\alpha) \partial\left(\mathbf{k}_{1}\left(\delta_{t}\right)\right) \widehat{\otimes} \delta_{\bar{x}}\right)=\varphi(\alpha) \partial_{\varphi, s}\left(1 \widehat{\otimes}_{\mathbf{k}_{1}} \delta_{h(t, \bar{x})}\right) .
$$

The claimed (70) follows from (71), (73) and (74).

The following result implies, in view of the equality $I_{\varphi} \widehat{\otimes}_{\mathbf{k}_{1}} \mathcal{D}(X)=\operatorname{ker}\left(\varphi_{*}\right) \subset \mathcal{D}_{\mathbf{k}_{1}}(X)$ of Theorem 7.7, that $\partial_{\varphi, s \mid \operatorname{ker}\left(\varphi_{*}\right)}$ does not depend on the choice of $s$.

Corollary 7.12. We have $\partial_{\varphi, s}=\partial_{\varphi}: I_{\varphi} \widehat{\otimes}_{\mathbf{k}_{1}} \mathcal{D}(X)=\operatorname{ker}\left(\varphi_{*}\right) \stackrel{\partial_{\varphi}}{\rightarrow} \mathcal{D}_{\mathbf{k}_{2}}(X)$.

Proof. Since the $K$-linear span of the elements $\alpha \widehat{\otimes}_{\mathbf{k}_{1}} \mu$ with $\alpha \in I_{\varphi}$ is dense in $I_{\varphi} \widehat{\otimes}_{\mathbf{k}_{1}} \mathcal{D}(X)$, it suffices to check that $\partial_{\varphi, s}\left(\alpha \widehat{\otimes}_{\mathbf{k}_{1}} \mu\right)=\partial_{\varphi}\left(\alpha \widehat{\otimes}_{\mathbf{k}_{1}} \mu\right)$ for these elements. According to Proposition 7.11 we have

$$
\partial_{\varphi, s}\left(\alpha \widehat{\otimes}_{\mathbf{k}_{1}} \mu\right)=\partial(\alpha) \varphi_{*}\left(1 \widehat{\otimes}_{\mathbf{k}_{1}} \mu\right)+\varphi(\alpha) \partial_{\varphi, s}\left(1 \widehat{\otimes}_{\mathbf{k}_{1}} \mu\right)=\partial(\alpha) \varphi_{*}\left(1 \widehat{\otimes}_{\mathbf{k}_{1}} \mu\right) .
$$

Thanks to $(61), \varphi_{*}\left(1 \widehat{\otimes}_{\mathbf{k}_{1}} \mu\right)=1 \widehat{\otimes}_{\mathbf{k}_{2}} \mu$ and then, by Lemma 7.9 ,

$$
\partial(\alpha) \varphi_{*}\left(1 \widehat{\otimes}_{\mathbf{k}_{1}} \mu\right)=\partial(\alpha) \widehat{\otimes}_{\mathbf{k}_{2}} \mu=\partial_{\varphi}\left(\alpha \widehat{\otimes}_{\mathbf{k}_{1}} \mu\right) .
$$

We end this subsection expressing the operator $\partial_{\varphi, s}$ on functions in terms of the usual derivative. Recall the locally analytic locally analytic function $j_{s}: X \rightarrow T$ characterized by the rule $x=j_{s}(x) s(\pi(x))$. Define

$$
\partial \log \mathbf{k}_{1}: T \longrightarrow \mathcal{O}_{2}
$$

by the rule

$$
\left(\partial \log \mathbf{k}_{1}\right)(t):=\frac{\partial\left(\mathbf{k}_{1}\left(\delta_{t}\right)\right)}{\varphi\left(\mathbf{k}_{1}\right)\left(\delta_{t}\right)}=\frac{\partial\left(\mathbf{k}_{1}\left(\delta_{t}\right)\right)}{\mathbf{k}_{2}\left(\delta_{t}\right)} .
$$


Consider the maps

$$
\begin{aligned}
& \partial_{*}: \mathcal{A}_{\mathbf{k}_{1}}(X) \longrightarrow \mathcal{A}\left(X, \mathcal{O}_{2}\right), \partial_{*}(F):=\partial \circ F \\
& \varphi_{*}: \mathcal{A}_{\mathbf{k}_{1}}(X) \longrightarrow \mathcal{A}_{\mathbf{k}_{2}}(X), \varphi_{*}(F):=\varphi \circ F .
\end{aligned}
$$

Lemma 7.13. If $F \in \mathcal{A}_{\mathbf{k}_{1}}(X)$, then

$$
\partial_{*}(F)=\epsilon_{s, \partial, \mathbf{k}_{1}, \varphi_{*}(F)}+\partial_{\varphi, s}(F)
$$

where $\epsilon:=\epsilon_{s, \partial, \mathbf{k}_{1}, \varphi_{*}(F)}$ is the function

$$
\epsilon(x)=\left(\partial \log \mathbf{k}_{1}\right)\left(j_{s}(x)\right) \varphi_{*}(F)(x)
$$

on $X$.

We end this section with the following remark. Suppose that $a: T \times X \rightarrow X$ has the property that there exists a set $I$ such that $X=\bigsqcup_{i \in I} X_{i}$, each $X_{i}$ being $T$-stable. Then

$$
\bar{X}:=T \backslash X=\bigsqcup_{i \in I} T \backslash X_{i}=: \bigsqcup_{i \in I} \bar{X}_{i}
$$

We say that $X$ is a locally trivial $T$-bundle if every $X_{i} \rightarrow \bar{X}_{i}$ is a trivial $T$-bundle, i.e. $\bar{X}_{i}$ has the structure of a locally compact, paracompact, $p$-adic manifold and there are locally analytic sections $s_{i}: \bar{X}_{i} \rightarrow X_{i}$ such that the locally analytic maps $h_{i}=h_{s_{i}}: T \times \bar{X}_{i} \rightarrow X_{i}$ given by $h_{i}(t, \bar{x}):=t s_{i}(\bar{x})$ are locally analytic isomorphisms. We call the triple $(T, X, \mathcal{S})$ with $\mathcal{S}:=\left\{s_{i}: \bar{X}_{i} \rightarrow X_{i}\right\}$ a split locally trivial $T$-bundle. This notion isn't a generalization of what we have proved so far because we could give $\bar{X}$ the structure of a locally compact, paracompact, $p$-adic manifold making the $\bar{X}_{i}$ s open in it and then define $s: \bar{X} \rightarrow X$ by the rule $s(\bar{x}):=s_{i}(\bar{x})$ if $\bar{x} \in \bar{X}_{i}$, which is indeed locally analytic and realizes $X \rightarrow \bar{X}$ as a trivial $T$-bundle.

7.3. The relation with the Coleman-Teitelbaum cocycle. In this subsection we will apply the notions and results of the previous $\S 7.1$ and $\S 7.2$ as follows. We take $X=W, T=\mathbb{Q}_{p}^{\times}$, and let $\pi: X \rightarrow \bar{X}$ be the map $\pi: W \rightarrow \mathbb{P}^{1}\left(\mathbb{Q}_{p}\right)$ defined by $\pi(x, y):=[x: y]$. To see that the above results apply, we explain why this map is a trivial $T$-bundle. By the remark at the end of $\S 7.2$, it suffices to show that $\pi$ is a locally trivial $\mathbb{Q}_{p}^{\times}$-bundle. To this end, let us identify $\mathbb{Q}_{p} \subset \mathbb{P}^{1}\left(\mathbb{Q}_{p}\right)$ with the set of elements of the form $z=[z: 1]$, so that $\pi(x, y)=x / y \in \mathbb{Q}_{p}$ for $y \neq 0$ and $\pi(x, 0)=\infty$. Set $\bar{X}_{0}:=\mathbb{Z}_{p}, \bar{X}_{\infty}:=\mathbf{P}^{1}\left(\mathbb{Q}_{p}\right)-\mathbb{Z}_{p}$ and $X_{i}:=\pi^{-1}\left(\bar{X}_{i}\right)$, which are $\mathbb{Q}_{p}^{\times}$-stable by construction. Explicitly, we have

$$
\begin{aligned}
X_{0} & =\left\{(x, y): y \neq 0 \text { and } x / y \in \mathbb{Z}_{p}\right\}, \\
X_{\infty} & =\left\{(x, y): y \neq 0 \text { and } x / y \in \mathbb{Q}_{p}-\mathbb{Z}_{p}\right\} \cup\{(x, 0)\} \\
& =\left\{(x, y): x \neq 0 \text { and } y / x \in p \mathbb{Z}_{p}\right\} .
\end{aligned}
$$

Then the functions $s_{0}([z: 1]):=(z, 1)$ for $[z: 1] \in \bar{X}_{0}$ and $s_{\infty}([1: z])=(1, z)$ for $[1: z] \in \bar{X}_{\infty}$ do the job. From now on we fix an integer $k \geq 0$.

Let us write the elements of $\mathbb{Q}_{p}^{\times}\left(\right.$resp. $\left.\mathbb{Z}_{p}^{\times}\right)$uniquely in the form $t=p^{\operatorname{ord}_{p}(t)}[t]\langle t\rangle$ (resp. $t=[t]\langle t\rangle$ ) using the decomposition $\mathbb{Q}_{p}^{\times}=p^{\mathbb{Z}} \times \mathbb{F}_{p}^{\times} \times\left(1+p \mathbb{Z}_{p}\right)\left(\right.$ resp. $\left.\mathbb{Z}_{p}^{\times}=\mathbb{F}_{p}^{\times} \times\left(1+p \mathbb{Z}_{p}\right)\right)$. Suppose that $\mathcal{O}$ is an affinoid $E$-algebra, write $\mathcal{A}_{k}(W)$ for the space of $E$-valued locally analytic functions on $W$ such that $F(p x, p y)=p^{k} F(x, y)$ and let $\mathcal{D}_{k}(W)$ be its strong dual. Then $\mathcal{D}_{k}(W)$ is naturally a $\mathcal{D}\left(\mathbb{Z}_{p}^{\times}\right)$-module and, for a continuous character $\omega: \mathbb{Z}_{p}^{\times} \rightarrow \mathcal{O}^{\times}$, we may form

$$
\mathcal{D}_{\omega, k}(W):=\mathcal{O} \widehat{\otimes}_{\omega} \mathcal{D}_{k}(W) .
$$

Alternatively, if $\mathbf{k}: \mathbb{Q}_{p}^{\times} \rightarrow \mathcal{O}^{\times}$is the character $\mathbf{k}(t)=p^{k \operatorname{ord}_{p}(t)} \omega([t]\langle t\rangle)$, then $\mathcal{D}_{\omega, k}(W)=\mathcal{D}_{\mathbf{k}}(W)$ (this is an easy application of Proposition 7.6).

Because $\mathbf{H o m}_{\text {cts }}\left(1+p \mathbb{Z}_{p}, \mathbf{G}_{m}\right) \simeq \mathfrak{S p}(E\langle S\rangle)$ if we work over the field $E$ (a finite extension of $\mathbb{Q}_{p}$ ), there is an open affinoid neighbourhood $\Omega=\Omega_{k} \subset \mathbf{H o m}_{c t s}\left(\mathbb{Z}_{p}^{\times}, \mathbf{G}_{m}\right)$ of $k$ which parametrizes the characters of the form $t \mapsto[t]^{k}\langle t\rangle^{s}$. The inclusion $\Omega \subset \mathbf{H o m}_{c t s}\left(\mathbb{Z}_{p}^{\times}, \mathbf{G}_{m}\right)$ corresponds to a continuous character $\omega=\omega_{k}: \mathbb{Z}_{p}^{\times} \rightarrow \mathcal{O}^{\times}$with $\mathcal{O}:=\mathcal{O}(\Omega)$. Setting $t^{\mathbf{k}}:=p^{k \operatorname{ord}_{p}(t)} \omega([t]\langle t\rangle)$ defines a continuous character $\mathbf{k}: \mathbb{Q}_{p}^{\times} \rightarrow \mathcal{O}^{\times}$which parametrizes the characters of the form $t \mapsto p^{\operatorname{ord}_{p}(t)}[t]^{k}\langle t\rangle^{s}$. Writing $\varphi: \mathbf{k} \rightarrow k$ for the evaluation at $s=k$ specialization map, we have $\partial:=\left.\frac{d}{d s}\right|_{s=k} \in \operatorname{Der}(\varphi)$. We will apply the results of $\S 7.1$ and $\S 7.2$ with $K=E$ and $\mathbf{k}_{1}=\mathbf{k} \rightarrow k=\mathbf{k}_{2}$. 
Let $\mathcal{C}\left(\mathcal{V}, \mathcal{D}_{\mathbf{k}}(W)\right)^{\widetilde{\Gamma}_{N^{+}, N^{-}}}$be the space of $\widetilde{\Gamma}_{N^{+}, N^{-}}$-invariant maps $c: \mathcal{V} \rightarrow \mathcal{D}_{\mathbf{k}}(W)$ (see [34, Proposition 3.5] for a description in terms of lattices and note the twist of the action in the weight $k>0$ case) and suppose that $\mu_{\phi} \in \mathcal{D}^{k}\left(\mathbb{P}^{1}\left(\mathbb{Q}_{p}\right)\right)^{\Gamma_{N^{+}, N^{-}}}$is $p$-new, as in the introduction, of higher even weight $k+2 \geq 2$. Here, $\mathcal{D}^{k}\left(\mathbb{P}^{1}\left(\mathbb{Q}_{p}\right)\right)$, the higher weight analogous of the space $\mathcal{D}^{0}\left(\mathbb{P}^{1}\left(\mathbb{Q}_{p}\right)\right)$, is defined as follows. Write $\mathcal{A}\left(\mathbb{P}^{1}\left(\mathbb{Q}_{p}\right)\right)^{k} \subset \mathcal{A}\left(\mathbb{Q}_{p}\right)$ for the space of locally analytic functions which may have a pole of order $\leq k$ at $\infty$ (see [28, Definition 3.2], for example). Writing $\mathbf{P}_{k}$ for the space of two variable, homogeneous polynomials of degree $k$ with coefficients in $E$, we have $\mathbf{P}_{k} \subset \mathcal{A}\left(\mathbb{P}^{1}\left(\mathbb{Q}_{p}\right)\right)^{k}$. We let $\mathcal{D}\left(\mathbb{P}^{1}\left(\mathbb{Q}_{p}\right)\right)^{k}$ be the $E$-dual of $\mathcal{A}\left(\mathbb{P}^{1}\left(\mathbb{Q}_{p}\right)\right)^{k}$ and write $\mathcal{D}^{k}\left(\mathbb{P}^{1}\left(\mathbb{Q}_{p}\right)\right)$ for the subspace of those locally analytic distributions $\mu \in \mathcal{D}\left(\mathbb{P}^{1}\left(\mathbb{Q}_{p}\right)\right)^{k}$ such that $\mu(P)=0$ for every $P \in \mathbf{P}_{k}$. Then there exists a family $\mu_{\mathbf{k}, *}:=\left\{\mu_{\mathbf{k}, v}\right\}_{v \in \mathcal{V}}$ with the property that, writing $\mu_{k, v}$ for the specialization of $\mu_{\mathbf{k}, v}$ at $k$ obtained by $\varphi$, we have, as in (5),

$$
\mu_{k, v}:=\varphi_{*}\left(\mu_{\mathbf{k}, v}\right)=\mu_{\phi} \text { for every } v \in \mathcal{V}^{+} .
$$

(See [4, Theorem 2.5 and Lemma 2.12] and [34, Theorem 3.7, the subsequent discussion and Proposition $3.8])$.

Let $\mathbf{V}_{k}$ be the $E$-dual of $\mathbf{P}_{k}$. Given the family $\mu_{\mathbf{k}, v} \in \mathcal{C}\left(\mathcal{V}, \mathcal{D}_{\mathbf{k}}(W)\right)^{\widetilde{\Gamma}_{N^{+}, N^{-}}}$as above such that $\mu_{k, v}=\mu_{\phi}$ and $\tau \in \mathcal{H}_{p}^{\text {ur }}$ such that $r(\tau) \in \mathcal{V}^{+}$, we define

$$
c_{\tau}^{\mu_{\mathbf{k}, *}}(\gamma)(P):=\partial_{s, \varphi}\left(\mu_{\mathbf{k}, r(\tau)}\right)(P)-\partial_{s, \varphi}\left(\mu_{\mathbf{k}, \gamma r(\tau)}\right)(P), \gamma \in \Gamma_{N^{+}, N^{-}} \text {and } P \in \mathbf{P}_{k} .
$$

On the other hand, we recall that, thanks to Teitelbaum's Poisson kernel formula (see [44]), the Coleman cocycle attached to $\phi$ can be expressed by the formula

$$
P_{\tau}\left(\mu_{\phi}\right)(\gamma):=\int_{W} \log \left(\frac{x-\gamma \tau y}{x-\tau y}\right) P(x, y) d \mu_{\phi}(x, y) .
$$

We recall from the introduction that $\mu_{\phi} \in \mathcal{D}^{0}\left(\mathbb{P}^{1}\left(\mathbb{Q}_{p}\right)\right)^{\Gamma_{N^{+}, N^{-}}}$is zero on $\mathbf{P}_{k}$.

Proposition 7.14. We have $c_{\tau}^{\mu_{\mathbf{k}, *}} \in Z^{1}\left(\Gamma_{N^{+}, N^{-}}, \mathbf{V}_{k}\right)$ and $c_{\tau}^{\mu_{\mathbf{k}, *}}=P_{\tau}\left(\mu_{\phi}\right)$ in $H^{1}\left(\Gamma_{N^{+}, N^{-}}, \mathbf{V}_{k}\right)$.

Proof. Set $\Theta_{\tau, P}(x, y):=\langle x-\tau y\rangle^{s-k} P(x, y) \in \mathcal{A}_{\mathbf{k}}(W)^{8}$, so that $\partial_{*}\left(\Theta_{\tau, P}\right)=\log (x-\tau y) P(x, y)=: \theta_{\tau, P}$ and then, by Lemma 7.13 ,

$$
\partial_{\varphi, s}\left(\Theta_{\tau, P}\right)=\partial_{*}\left(\Theta_{\tau, P}\right)-\epsilon_{s, \partial, \omega, \varphi\left(\Theta_{\tau, P}\right)}=\theta_{\tau, P}-\epsilon_{s, \partial, \omega, P} .
$$

(For the last equality, notice that $\varphi\left(\Theta_{\tau, P}\right)=P$ ). Then we see that, by Proposition 7.10,

$$
\begin{aligned}
\partial\left(\mu_{\mathbf{k}, v}\left(\Theta_{\tau, P}\right)\right) & =\partial_{\varphi, s}\left(\mu_{\mathbf{k}, v}\right)\left(\varphi\left(\Theta_{\tau, P}\right)\right)+\varphi_{*}\left(\mu_{\mathbf{k}, v}\right)\left(\partial_{\varphi, s}\left(\Theta_{\tau, P}\right)\right) \\
& =\partial_{\varphi, s}\left(\mu_{\mathbf{k}, v}\right)(P)+\mu_{\phi}\left(\theta_{\tau, P}-\epsilon_{s, \partial, \omega, P}\right) .
\end{aligned}
$$

Define a function $J: \mathcal{H}_{p}^{u r} \rightarrow \mathbf{V}_{k}$ by the rule $J(\tau)(P):=\partial\left(\mu_{\mathbf{k}, r(\tau)}\left(\Theta_{\tau, P}\right)\right)$. Then one checks that, since $\varphi_{*}\left(\mu_{\mathbf{k}, v}\right)=\mu_{\phi}$ is zero on $\mathbf{P}_{k}$ (by $p$-newness of $\left.\phi\right), J(\gamma \tau)(P)=(\gamma J(\tau))(P)$, so that

$$
b_{\tau}(\gamma):=J(\gamma \tau)-J(\tau)=\gamma J(\tau)-J(\tau) \in B^{1}\left(\Gamma, \mathbf{V}_{k}\right) .
$$

\footnotetext{
${ }^{8}$ We have indeed

$$
\begin{aligned}
\Theta_{\tau, P}(t x, t y) & :=\langle t x-\tau t y\rangle^{s-k} P(t x, t y)=t^{k}\langle t\rangle^{s-k}\langle x-\tau y\rangle^{s-k} P(x, y) \\
& =p^{k \operatorname{ord}_{p}(t)}[t]^{k}\langle t\rangle^{k}\langle t\rangle^{s-k}\langle x-\tau y\rangle^{s-k} P(x, y) \\
& =p^{k \operatorname{ord}_{p}(t)}[t]^{k}\langle t\rangle^{s}\langle x-\tau y\rangle^{s-k} P(x, y)=t^{\mathbf{k}} \Theta_{\tau, P}(x, y) .
\end{aligned}
$$
}


Writing $(76)_{\tau, v}$ for the equation (76) applied to $(\tau, v)$, we see that

$$
\begin{aligned}
b_{\tau}(\gamma)(P)= & (76)_{\gamma \tau, r(\gamma \tau)=\gamma r(\tau)}-(76)_{\tau, r(\tau)} \\
= & \partial_{\varphi, s}\left(\mu_{\mathbf{k}, \gamma r(\tau)}\right)(P)+\mu_{\phi}\left(\theta_{\gamma \tau, P}-\epsilon_{s, \partial, \omega, P}\right) \\
& -\partial_{\varphi, s}\left(\mu_{\mathbf{k}, r(\tau)}\right)(P)-\mu_{\phi}\left(\theta_{\tau, P}-\epsilon_{s, \partial, \omega, P}\right) \\
= & \left(\partial_{\varphi, s}\left(\mu_{\mathbf{k}, \gamma r(\tau)}\right)-\partial_{\varphi, s}\left(\mu_{\mathbf{k}, r(\tau)}\right)\right)(P) \\
& +\mu_{\phi}\left(\theta_{\gamma \tau, P}-\epsilon_{s, \partial, \omega, P}+\epsilon_{s, \partial, \omega, P}-\theta_{\tau, P}\right) \\
= & -c_{\tau}^{\mu_{\mathbf{k}, *}}(\gamma)(P)+\mu_{\phi}\left(\theta_{\gamma \tau, P}-\theta_{\tau, P}\right) .
\end{aligned}
$$

But we have

$$
\begin{aligned}
\theta_{\gamma \tau, P}-\theta_{\tau, P} & =\log (x-\gamma \tau y) P(x, y)-\log (x-\tau y) P(x, y) \\
& =\log \left(\frac{x-\gamma \tau y}{x-\tau y}\right) P(x, y)
\end{aligned}
$$

so that

as claimed.

$$
\mu_{\phi}\left(\theta_{\gamma \tau, P}-\theta_{\tau, P}\right)=P_{\tau}\left(\mu_{\phi}\right)
$$

\section{REFERENCES}

[1] F. Andreatta, A. Iovita and G. Stevens, Overconvergent modular sheaves and modular forms for $G L_{2 / F}$, Israel J. Math. 201, 299-359.

[2] F. Andreatta, A. Iovita and G. Stevens, Overconvergent Eichler-Shimura isomorphisms. To appear in J. Inst. Math. Jussieu, available on CJO2014. doi:10.1017/S1474748013000364.

[3] A. Ash and G. Stevens, p-adic deformation of arithmetic cohomology, draft dated 29.09.2008.

[4] M. Bertolini and H. Darmon, Hida families and rational points on elliptic curves, Invent. Math. 168 (2007), no. 2, $371-431$.

[5] M. Bertolini, H. Darmon and A. Iovita, Families of automorphic forms on definite quaternion algebras and Teitelbaum's conjecture, Astérisque 331 (2010), 29-64.

[6] S. Bosch, W. Lutkebohmert and M. Raynaud, Néron models, Springer-Verlag, 1990.

[7] N. Bourbaki, Groupes et Algébres de Lie, Hermann, Paris, 1972.

[8] K. S. Brown, Cohomology of groups, Springer, 1980.

[9] K. Buzzard, On p-adic families of automorphic forms. In: Modular Curves and Abelian Varieties, 23-44, Progr. Math., vol. 224, Birkhäuser Verlag, Basel (2004).

[10] R. F. Coleman, p-adic Banach spaces and families of modular forms, Invent. Math. 127 (3), 417-479.

[11] R. F. Coleman and B. Mazur, The eigencurve. In: Galois representations in arithmetic algebraic geometry (Durham, 1996), 1-113, London Math. Soc. Lecture Note Ser. 254, Cambridge Univ. Press.

[12] H. Darmon, Integration on $\mathcal{H}_{p} \times \mathcal{H}$ and arithmetic applications, Ann. of Math. 154 (2001), no. 2, 589-639.

[13] H. Darmon, Rational points on modular elliptic curves, CBMS Regional Conference Series in Mathematics, 101. Published for the Conference Board of the Mathematical Sciences, Washington, DC; by the American Mathematical Society, Providence, RI, 2004.

[14] S. Dasgupta, Gross-Stark units, Stark-Heegner points, and class fields of real quadratic fields, PhD thesis.

[15] S. Dasgupta, Stark-Heegner points on modular Jacobians, Ann. Scient. Ec. Norm. Sup. 38 (2005), 427-469.

[16] S. Dasgupta and M. Greenberg, $\mathcal{L}$-invariants and Shimura curves, to appear in Algebra and Number Theory.

[17] S. Dasgupta and J. Teitelbaum, The p-adic upper half plane. In: p-adic Geometry, Lectures from the 2007 Arizona Winter School, ed. D. Savitt, D. Thakur. University Lecture Series 45, Amer. Math. Soc., Providence, RI, 2008.

[18] J. Fresnel and M. van der Put, Rigid analytic geometry and its applications, Birkhäuser Boston, 2004.

[19] M. Greenberg, Stark-Heegner points and the cohomology of quaternionic Shimura varieties, Duke Math. J. 147 (2009), no. $3,541-575$.

[20] M. Greenberg and M. A. Seveso, p-adic families of cohomological modular forms for indefinite quaternion algebras and the Jacquet-Langlands correspondence. To appear in Canad. J. Math., doi: 10.4153/CJM-2015-062-x.

[21] M. Greenberg, M. A. Seveso and S. Shahabi, Modular p-adic L-functions attached to real quadratic fields and arithmetic applications. To appear in J. Reine Angew. Math., doi: 10.1515 /crelle-2014-0088.

[22] R. Greenberg, G. Stevens, p-adic L-functions and p-adic periods of modular forms, Invent. Math. 111 (1993), $407-447$.

[23] H. Hida, Galois representations into $G L_{\mathbf{2}}\left(\mathbb{Z}_{p}[[X]]\right)$ attached to ordinary cusp forms, Invent. Math. 85 (1986), 545-613.

[24] H. Hida, Iwasawa modules attached to congruences of cusp forms, Ann. Scient. Ec. Norm. Sup. 19 (1986), $231-273$.

[25] M. Longo and S. Vigni, The rationality of quaternionic Darmon points over genus fields of real quadratic fields, IMRN 2014 (2014), 3632-3691. 
[26] M. Longo, V. Rotger and S. Vigni, On rigid analytic uniformizations of Jacobians of Shimura curves, Amer. J. Math. 135 (2012), 1197-1246.

[27] B. Mazur, J. Tate, J. Teitelbaum, On p-adic analogues of the conjecture of Birch and Swinnerton-Dyer, Invent. Math. 84 (1986), 1-48.

[28] Rotger V., Seveso M. A., L-invariants and Darmon cycles attached to modular forms, J. Eur. Math. Soc. 14 (2012), Issue 6, 1955-1999.

[29] J-P. Serre, Formes modulaires et fonctions zeta p-adiques. In: Modular functions of one variable III, Lecture Notes in Mathematics 350, Springer, 191-268.

[30] J.-P. Serre, Local fields, Springer, 1979.

[31] J.-P. Serre, Trees, Springer, 1980.

[32] J. P. Serre, Lie algebras and Lie groups, Springer-Verlag, 1964.

[33] Seveso, M. A., p-adic L-functions and the rationality of Darmon cycles, Canad. J. Math. 64 (5) (2012), $1122-1181$.

[34] Seveso, M. A., Heegner cycles and derivatives of p-adic L-functions, J. Reine Angew. Math. 686 (2014), 111-148.

[35] Seveso, M. A., The Teitelbaum conjecture in the indefinite setting, Amer. J. Math. 135 no. 6 (2013), $1525-1557$.

[36] M. Papikian, Rigid-analytic geometry and the uniformization of abelian varieties. In: Snowbird lectures in algebraic geometry, R. Vakil (ed.), Contemp. Math. 388, AMS Providence, 2005, 145-160.

[37] E. de Shalit, Eichler cohomology and periods of modular forms on p-adic Schottky groups, J. Reine Angew. Math. 400 (1989), 3-31.

[38] P. Schneider, Nonarchimedean Functional Analysis, Springer Monographs in Mathematics. Springer-Verlag, Berlin, 2002.

[39] P. Schneider, p-adic analysis and Lie groups, available at http://wwwmath.uni-muenster.de/u/pschnei/publ/lectnotes/padic-analysis.pdf.

[40] P. Schneider and J. Teitelbaum, Locally analytic distributions and p-adic representation theory, with applications to $\mathbf{G L}_{2}$, J. Amer. Math. Soc. 15 (2002), 443-468.

[41] P. Schneider and J. Teitelbaum, Continuous and locally analytic representation theory, available at http://www.cms.zju.edu.cn/UploadFiles/AttachFiles/20048416190427.pdf (2004).

[42] G. Stevens, Rigid analytic modular symbols. Preprint dated April 21, 1994.

[43] G. Stevens, Coleman's $\mathcal{L}$-invariant and families of modular forms, Asterisque 331 (2010), 1-12.

[44] J. Teitelbaum, Values of p-adic L-functions and a p-adic Poisson kernel, Invent. Math. 101 (1990), 395-410.

E-mail address: mgreenbe@ucalgary.ca

Department of Mathematics and Statistics, University of Calgary, 2500 University Drive NW, Calgary, AlBerta, T2N 1N4, CANADA

E-mail address: seveso.marco@gmail.com

Dipartimento di Matematica, Università di Milano, Via Cesare Saldini 50, 20133 Milano, Italia

Department of Mathematics and Statistics, McGill University, 805 Sherbrooke St. West, Montreal, Quebec, H3A 2K6, CANADA 